\title{
Flavour Theory: 2009
}

\author{
Andrzej J. Buras* \\ Technical University Munich, Physics Department, D-85748 Garching, Germany, \\ TUM Institute for Advanced Study, D-80333 München, Germany \\ E-mail: aburaseph.tum.de
}

\begin{abstract}
After an overture and a non-technical exposition of the relevant theoretical framework including a brief discussion of some of the most popular extensions of the Standard Model, we will compile a list of 20 goals in flavour physics that could be reached already in the next decade. In addition to $K, D$ and $B_{s, d}$ decays and lepton flavour violation also flavour conserving observables like electric dipole moments of the neutron and leptons and $(g-2)_{\mu}$ are included in this list. Flavour violation in high energy processes is also one of these goals. Subsequently we will discuss in more detail some of the most urgent issues for the coming years in the context of several extensions of the Standard Model like models with Minimal Flavour Violation, the general MSSM, the Littlest Higgs Model with T parity, Randall-Sundrum models and supersymmetric flavour models. This presentation is not meant to be a comprehensive review of flavour physics but rather a personal view on this fascinating field and an attempt to collect those routes that with the help of upcoming experiments should allow us to reach a much deeper understanding of physics, in particular flavour physics, at very short distance scales.
\end{abstract}

European Physical Society Europhysics Conference on High Energy Physics, EPS-HEP 2009,

July 16 - 222009

Krakow, Poland

${ }^{*}$ Speaker. 


\section{Overture}

The main goal of elementary particle physics is to search for physics laws at very short distance scales. From the Heisenberg uncertainty principle [1] we know that to test scales of order $10^{-18} \mathrm{~m}$ we need the energy of approximately $200 \mathrm{GeV}$. With approximately $E=4 \mathrm{TeV}$, effectively available at the LHC, we will be able to probe distances as short as $5 \cdot 10^{-20} \mathrm{~m}$. Unfortunately, it is unlikely that we can do better before 2046 through high energy collider experiments. On the other hand flavour-violating and CP-violating processes are very strongly suppressed and are governed by quantum fluctuations that allow us to test energy scales as high as $200 \mathrm{TeV}$ corresponding to short distances in the ballpark of $10^{-21} \mathrm{~m}$. Even shorter distance scales can be tested, albeit indirectly, in this manner. Consequently frontiers in testing ultrashort distance scales belong to flavour physics or more concretely to very rare processes like particle-antiparticle mixing, rare decays of mesons, CP violation and lepton flavour violation. Also electric dipole moments and $(g-2)_{\mu}$ belong to these frontiers even if they are flavour conserving. While such tests are not limited by the available energy, they are limited by the available precision. The latter has to be very high as the Standard Model (SM) has been until now very successful and finding departures from its predictions has become a real challenge.

Flavour physics developed over the last two decades into a very broad field. In addition to $K, D$ and $B_{d}$ decays and $K^{0}-\bar{K}^{0}$ and $B_{d}-\bar{B}_{d}$ mixings that were with us for quite some time, $B_{s}^{0}-\bar{B}_{s}^{0}$ mixing, $B_{s}$ decays and $D^{0}-\bar{D}^{0}$ mixing belong these days to the standard repertoire of any flavour workshop. Similarly lepton flavour violation (LFV) gained in importance after the discovery of neutrino oscillations and related non-vanishing neutrino masses even if within the SM the LFV is basically unmeasurable. Simultaneously new ideas for the explanation of the quark and lepton mass spectra and the related weak mixings, summarized by the CKM $[2,3]$ and PMNS $[4,5]$ matrices, developed significantly in this decade. Moreover the analyses of electric dipole moments (EDM's), of the $(g-2)_{\mu}$ anomaly and of flavour changing neutral current (FCNC) processes in top quark decays intensified during the last years in view of the related experimental progress that is expected to take place in the next decade.

The correlations between all these observables and the interplay of flavour physics with direct searches for new physics (NP) and electroweak precision studies will tell us hopefully one day which is the proper extension of the SM.

In preparing this talk I have been guided by the impressive success of the CKM picture of flavour changing interactions [2,3], evident in the excellent talks of Adrian Bevan [6] and Giovanni Punzi, and also by several tensions between the flavour data and the SM that possibly are the first signs of NP. Fortunately, there is still a lot of room for NP contributions, in particular in rare decays of mesons and charged leptons, in CP-violating transitions and in electric dipole moments of leptons, of the neutron and of other particles. There is also a multitude of models that attempt to explain the existing tensions and to predict what experimentalists should find in the coming decade. Yet, in my opinion, those models should be favoured at present that try to address the important open questions of contemporary particle physics like the issue of the stabilization of the Higgs mass under loop corrections and the question of the origin of the observed hierarchies in fermion masses and mixings. Such extensions will play the dominant role in this report.

There is also the important question whether the footprints of NP that is responsible for the 
hierarchies in question will be seen directly at the LHC and indirectly through flavour and CPviolating processes in the coming decade. Hoping that this is indeed the case we will assume in what follows that the NP scales in various extensions of the SM discussed below are not larger that $2-3 \mathrm{TeV}$, so that the new particles predicted by these extensions are in the reach of the LHC.

After a brief recollection of the theoretical framework and the description of the most popular NP scenarios in Section 2, we will list in Section 3, the twenty most important goals in this field for the coming decade. There is no space to discuss all these goals in detail here. Therefore in Section 4 we will only discuss the ones which in my opinion are the most important at present. A number of enthusiastic statements will end this report.

I should strongly emphasize that I do not intend to present here a totally comprehensive review of flavour physics. Comprehensive reviews, written by a hundred of flavour experts are already present on the market [7-9] and moreover, extensive studies of the physics at future flavour machines and other visions can be found in $[10,11]$. I would rather like to paint a picture of flavour physics in general terms and collect various strategies for the exploration of this fascinating field that hopefully will turn out to be useful in the coming years. In this context I will recall present puzzles in flavour physics that could turn out to be the first hints of NP and on various occasions I will present the predictions of the NP scenarios mentioned in the Abstract. Last but certainly not least let me cite two excellent text books on CP violation and flavour physics [12,13], where many fundamentals of this field are clearly explained and other extensions of the SM and other observables are discussed in detail.

\section{Theoretical Framework}

\subsection{Preliminaries}

The starting point of any serious analysis of weak decays in the framework of a given extension of the SM is the basic Lagrangian

$$
\mathscr{L}=\mathscr{L}_{\mathrm{SM}}\left(g_{i}, m_{i}, V_{\mathrm{CKM}}^{i}\right)+\mathscr{L}_{\mathrm{NP}}\left(g_{i}^{\mathrm{NP}}, m_{i}^{\mathrm{NP}}, V_{\mathrm{NP}}^{i}\right),
$$

where $\left(g_{i}, m_{i}, V_{\mathrm{CKM}}^{i}\right)$ denote the parameters of the $\mathrm{SM}$ and $\left(g_{i}^{\mathrm{NP}}, m_{i}^{\mathrm{NP}}, V_{\mathrm{NP}}^{i}\right) \equiv \rho_{\mathrm{NP}}$ the additional parameters in a given NP scenario.

Our main goal then is to identify in weak decays the effects decribed by $\mathscr{L}_{\mathrm{NP}}$ in the presence of the background from $\mathscr{L}_{\mathrm{SM}}$. In the first step one derives the Feynman rules following from (2.1), which allows to calculate Feynman diagrams. But then we have to face two challenges:

- our theory is formulated in terms of quarks, but experiments involve their bound states: $K_{L}$, $K^{ \pm}, B_{d}^{0}, B_{s}^{0}, B^{ \pm}, B_{c}, D, D_{s}$, etc.

- NP takes place at very short distance scales $10^{-19}-10^{-18} \mathrm{~m}$, while $K_{L}, K^{ \pm}, B_{d}^{0}, B_{s}^{0}, B^{ \pm}$and other mesons live at $10^{-16}-10^{-15} \mathrm{~m}$.

The solution to these challenges is well known. One has to construct an effective theory relevant for experiments at low energy scales. Operator Product Expansion (OPE) and Renormalization Group (RG) methods are involved here. They allow to separate the perturbative short distance 
(SD) effects, where NP is present, from long distance (LD) effects for which non-perturbative methods are necessary. Moreover RG methods allow an efficient summation of large logarithms $\log \left(\mu_{\mathrm{SD}} / \mu_{\mathrm{LD}}\right)$. A detailed exposition of these techniques can be found in $[14,15]$ and fortunately we do not have to repeat them here. At the end of the day the formal expressions involving matrix elements of local operators and their Wilson coefficients can be cast into the following Master Formula for Weak Decays [16].

\subsection{Master Formula for Weak Decays}

The master formula in question reads:

$$
\mathrm{A}(\text { Decay })=\sum_{i} B_{i} \eta_{\mathrm{QCD}}^{i} V_{\mathrm{CKM}}^{i} F_{i}\left(m_{t}, \rho_{\mathrm{NP}}\right),
$$

where $B_{i}$ are non-perturbative parameters representing hadronic matrix elements of the contributing operators, $\eta_{i}^{\mathrm{QCD}}$ stand symbolically for the renormalization group factors, $V_{\mathrm{CKM}}^{i}$ denote the relevant combinations of the elements of the CKM matrix and finally $F_{i}\left(m_{t}, \rho_{\mathrm{NP}}\right)$ denote the loop functions resulting in most models from box and penguin diagrams but in some models also representing tree level diagrams if such diagrams contribute. The internal charm contributions have been suppressed in this formula but they have to be included in particular in $K$ decays and $K^{0}-\bar{K}^{0}$ mixing. $\rho_{\mathrm{NP}}$ denotes symbolically all parameters beyond $m_{t}$, in particular the set $\left(g_{i}^{\mathrm{NP}}, m_{i}^{\mathrm{NP}}, V_{\mathrm{NP}}^{i}\right)$ in $(2.1)$. It turns out to be useful to factor out $V_{\mathrm{CKM}}^{i}$ in all contributions in order to see transparently the deviations from Minimal Flavour Violation (MFV).

In the SM only a particular set of parameters $B_{i}$ is relevant as there are no right-handed charged current interactions, the functions $F_{i}$ are real and the flavour and CP-violating effects enter only through the CKM factors $V_{\mathrm{CKM}}^{i}$. This implies that the functions $F_{i}$ are universal with respect to flavour so that they are the same in the $K, B_{d}$ and $B_{s}$ systems. Consequently a number of observables in these different systems are strongly correlated with each other within the SM.

The simplest class of extensions of the SM are models with Constrained Minimal Flavour Violation (CMFV) [17-20]. They are formulated as follows:

- All flavour changing transitions are governed by the CKM matrix with the CKM phase being the only source of $\mathrm{CP}$ violation,

- The only relevant operators in the effective Hamiltonian below the weak scale are those that are also relevant in the SM.

This implies that relative to the SM only the values of $F_{i}$ are modified but their universal character remains intact. In particular they are real. Moreover, in cases where $F_{i}$ can be eliminated by taking certain combinations of observables, universal correlations between these observables for this class of models result. We will encounter some of these correlations in Section 4.

In more general MFV models [21-23] new parameters $B_{i}$ and $\eta_{i}^{\mathrm{QCD}}$, related to new operators, enter the game but the functions $F_{i}$ still remain real quantities as in the CMFV framework and do not involve any flavour violating parameters. Consequently the $\mathrm{CP}$ and flavour violating effects in these models are again governed by the CKM matrix. However, the presence of new operators makes this approach less constraining than the CMFV framework. We will discuss some other aspects of this approach below. 
In the simplest non-MFV models, the basic operator structure of CMFV models remains but the functions $F_{i}$ in addition to real SM contributions can contain new flavour parameters and new complex phases. Consequently the CKM matrix ceases to be the only source of flavour and CP violation.

Finally, in the most general non-MFV models, new operators (new $B_{i}$ parameters) contribute and the functions $F_{i}$ in addition to real SM contributions can contain new flavour parameters and new complex phases.

Obviously this classification of different classes of models corresponds to a $2 \times 2$ matrix but before presenting this matrix let us briefly discuss the essential ingredients in our master formula.

Clearly without a good knowledge of non-perturbative factors $B_{i}$ no precision studies of flavour physics will be possible unless the non-perturbative uncertainties can be reduced or even removed by taking suitable ratios of observables. In certain rare cases it is also possible to measure the relevant hadronic matrix elements entering rare decays by using leading tree level decays. Examples of such fortunate situations are certain mixing induced $\mathrm{CP}$ asymmetries and the branching ratios for $K \rightarrow \pi v \bar{v}$ decays. Yet, in many cases one has to face the direct evaluation of $B_{i}$. While lattice calculations, QCD-sum rules, Light-cone sum rules and large- $N$ methods made significant progress in the last 20 years, the situation is clearly not satisfactory and one should hope that new advances in the calculation of $B_{i}$ parameters will be made in the LHC era in order to adequately use improved data. Recently an impressive progress in calculating the parameter $\hat{B}_{K}$, relevant for $\mathrm{CP}$ violation in $K^{0}-\bar{K}^{0}$ mixing, has been made and we will discuss its implications in Section 4.

An important progress has also been made in organizing the dominant contributions in nonleptonic two-body $B$ meson decays and decays like $B \rightarrow V \gamma$ with the help of the QCD factorization approach, SCET and the Perturbative QCD approach.

Concerning the factors $\eta_{\mathrm{QCD}}^{i}$ an impressive progress has been made during the last 20 years. The 1990's can be considered as the era of NLO QCD calculations. Basically the NLO corrections to all relevant decays and transitions have been calculated already in the last decade [14], with a few exceptions, like the width differences $\Delta \Gamma_{s, d}$ in the $B_{s, d}^{0}-\bar{B}_{s, d}^{0}$ systems that were completed only in 2003 [24-26]. This decade can be considered as the era of NNLO calculations. In particular one should mention here the NNLO calculations of QCD corrections to $B \rightarrow X_{s} l^{+} l^{-}$[27-33], $K^{+} \rightarrow \pi^{+} v \bar{v}$ [34-36], and in particular to $B_{s} \rightarrow X_{s} \gamma$ [37] with the latter one being by far the most difficult one. Also important steps towards a complete calculation of NNLO corrections to nonleptonic decays of mesons have been made in [38].

The final ingredients of our master formula, in addition to $V_{\mathrm{CKM}}^{i}$ factors, are the loop functions $F_{i}$ resulting from penguin and box diagrams with the exchanges of the top quark, $W^{ \pm}, Z^{0}$, heavy new gauge bosons, heavy new fermions and scalars. They are known at one-loop level in several extensions of the SM, in particular in the two Higgs doublet model (2HDM), the littlest Higgs model without $\mathrm{T}$ parity (LH), the ACD model with one universal extra dimension (UED), the MSSM with MFV and non-MFV violating interactions, the flavour blind MSSM (FBMSSM), the littlest Higgs model with T-parity (LHT), $Z^{\prime}$-models, Randall-Sundrum (RS) models, left-right symmetric models, the model with the sequential fourth generation of quarks and leptons. Moreover, in the SM $\mathscr{O}\left(\alpha_{s}\right)$ corrections to all relevant one loop functions are known. It should also be stressed again that in the loop functions in our master formula one can conveniently absorb tree level FCNC contributions present in particular in RS models. 
After this symphony of names like FBMSSM, LH, LHT, RS let us explain them briefly by summarizing the most popular extentions of the SM.

\subsection{Minimal Flavour Violation}

We have already formulated what we mean by CMFV and MFV. Let us first add here that the models with CMFV generally contain only one Higgs doublet and the top Yukawa coupling dominates. On the other hand models with MFV in which the operator structure differs from the SM one contain two Higgs doublets and bottom and top Yukawa couplings can be of comparable size. A well known example is the MSSM with MFV and large $\tan \beta$. The MFV framework can be elegantly formulated with the help of global symmetries present in the limit of vanishing Yukawa couplings [22,23] and its implications can be studied efficiently with the help of spurion technology [21,39]. However, I will not enter this presentation here as it can be found in basically any paper that discusses MFV. Recent discussions of various aspects of MFV can be found in [40-45].

Here let us only stress that the MFV symmetry principle in itself does not forbid the presence of flavour blind CP violating sources [40,42-44,46-50]. Therefore, in particular, a MFV MSSM suffers from the same SUSY CP problem as the ordinary MSSM. Either an extra assumption or a mechanism accounting for a natural suppression of these CP-violating phases is desirable. The authors of [21] proposed the extreme situation where the SM Yukawa couplings are the only source of CPV. In contrast, recently in [45], such a strong assumption has been relaxed and the following generalized MFV ansatz has been proposed: the SUSY breaking mechanism is flavour blind and $\mathrm{CP}$ conserving and the breaking of CP only arises through the MFV compatible terms breaking the flavour blindness. That is, CP is preserved by the sector responsible for SUSY breaking, while it is broken in the flavour sector. While the generalized MFV ansatz still accounts for a natural solution of the SUSY CP problem, it also leads to peculiar and testable predictions in low energy $\mathrm{CP}$ violating processes [45].

The MFV approach is simple and offers an elegant explanation of the fact that the CKM framework works so well even if NP is required to be present at scales $\mathscr{O}(1 \mathrm{TeV})$. But one has to admit that it is a rather pessimistic approach to NP. The deviations from the SM expectations in $\mathrm{CP}$ conserving processes amount in the case of CMFV to at most $50 \%$ at the level of the branching ratios [51-53]. More generally in the MFV framework only in cases where scalar operators are becoming important and helicity suppression in decays like $B_{s} \rightarrow \mu^{+} \mu^{-}$is lifted, enhancements of the relevant branching ratios by more than a factor of two and even one order of magnitude relative to the SM are possible. However, independently of whether it is CMFV or MFV, the CP violation in this class of models is SM-like and in order to be able to distinguish among various models in this class high precision will be required which calls for experiments like Super-Belle, Super-B facility in Frascati and $K \rightarrow \pi v \bar{v}$ experiments like NA62 and KOTO.

One should also emphasize that MFV in the quark sector does not offer the explanation of the size of the observed baryon-antibaryon asymmetry in the universe (BAU) and it does not address the hierarchy problem related to the quadratic divergences in the Higgs mass. Similarly the hierarchies in the quark masses and quark mixing angles remain unexplained in this framework. For this reason there is still potential interest in non-MFV new physics scenarios to which we will now turn our attention. 


\subsection{Most Popular Non-MFV Extensions of the SM}

The search for NP at the $1 \mathrm{TeV}$ scale is centered already for three decades around the hierarchy problem, be it the issue of quadratic divergences in the Higgs mass, the disparity of the electroweak, GUT and Planck scales or the doublet-triplet splitting in the context of SU(5) GUTs. The three most popular directions which aim to solve at least some of these problems are as follows:

a) Supersymmetry (SUSY)

In this approach the cancellation of quadratic divergences in $m_{H}$ is achieved with the help of new particles of different spin-statistics than the SM particles: supersymmetric particles. For this approach to work, these new particles should have masses below $1 \mathrm{TeV}$, otherwise the fine tuning of parameters cannot be avoided. One of the important predictions of the simplest realization of this scenario, the MSSM with R-parity, is the light Higgs with $m_{H} \leq 130 \mathrm{GeV}$ and one of its virtues is its perturbativity up to the GUT scales.

The ugly feature of the General MSSM (GMSSM) is a large number of parameters residing dominantly in the soft sector that has to be introduced in the process of supersymmetry breaking. Constrained versions of the MSSM can reduce the number of parameters significantly. The same is true in the case of the MSSM with MFV. An excellent review on supersymmetry can be found in [54].

The very many new flavour parameters in the soft sector makes the GMSSM not very predictive and moreover this framework is plagued by flavour and CP problems: FCNC processes and EDM's are generically well above the experimental data and upper bounds, respectively. Moreover the GMSSM framework addressing primarily the gauge hierarchy problem and the quadratic divergences in the Higgs mass does not provide automatically the hierarchical pattern of quark and lepton masses and of the hierarchical pattern of their FCNC and CP-violating interactions.

Much more interesting from this point of view are supersymmetric flavour models (SF) with flavour symmetries that allow a simultaneous understanding of the flavour structures in the Yukawa couplings and in SUSY soft-breaking terms, adequately suppressing FCNC and CP-violating phenomena and solving SUSY flavour and CP problems. A recent detailed study of various SF models has been performed in [55]. We have analysed there the following representative scenarios in which NP contributions are characterized by:

i) The dominance of right-handed ( $\mathrm{RH})$ currents (abelian model by Agashe and Carone [56]),

ii) Comparable left- and right-handed currents with CKM-like mixing angles represented by the special version (RVV2) of the non abelian $S U$ (3) model by Ross, Velasco and Vives [57] as discussed recently in [58] and the model by Antusch, King and Malinsky (AKM) [59],

iii) The dominance of left-handed (LH) currents in non-abelian models [60] $(\delta \mathrm{LL})$.

Through a model-independent analysis we have found that these three scenarios predicting quite different patterns of flavour violation should give a good representation of most SF models discussed in the literature. Short summaries of our results can be found in [61,62].

In Section 4 we will mainly confine our presentation of predictions of supersymmetry to these SF models. However, we will also briefly encounter the MSSM with MFV in which new flavour blind but CP-violating phases are present. This FBMSSM framework has been discussed in [46-49] 
and last year in [50], where a number of correlations between various flavour conserving and flavour violating observables, both $\mathrm{CP}$-violating, has been pointed out.

Next, let us recall that the new particles in supersymmetric models, that is squarks, sleptons, gluinos, charginos, neutralinos, charged Higgs particles $H^{ \pm}$and additional neutral scalars can contribute to FCNC processes through virtual exchanges in box and penguin diagrams. Moreover, new sources of flavour and $\mathrm{CP}$ violation come from the misalignement of quark and squark mass matrices and similar new flavour and CP-violating effects are present in the lepton sector. Some of these effects can be strongly enhanced at large $\tan \beta$. Finally, in the MSSM a useful parametrization of the new effects is given by $\delta_{i j}^{A B}$ with $i, j=1,2,3$ and $A, B=L, R$ in the context of the so-called mass insertion approach $[63,64]$. However, it should be emphasized that in certain models, like supersymmetric flavour models, this approximation is not always accurate and exact diagonalization of squark mass matrices is mandatory in order to obtain meaningful results [55, 65].

\section{b) Little Higgs Models}

In this approach the cancellation of divergences in $m_{H}$ is achieved with the help of new particles of the same spin-statistics. Basically the SM Higgs is kept light because it is a pseudoGoldstone boson of a new spontaneously broken global symmetry. Thus the Higgs is protected by a global symmetry from acquiring a large mass, although in order to achieve this the weak gauge group has to be extended and the Higgs mass generation properly arranged (collective symmetry breaking). The dynamical origin of the global symmetry in question and the physics behind its breakdown is not specified. But in analogy to QCD one could imagine a new strong force at scales $\mathscr{O}(10 \mathrm{TeV})$ among new very heavy fermions that bind together to produce the SM Higgs. In this scenario the SM Higgs is analogous to the pion. At scales well below $10 \mathrm{TeV}$ the Higgs is considered as an elementary particle but at $10 \mathrm{TeV}$ its composite structure should be seen. At these high scales one will have to cope with non-perturbative strong dynamics, and an unknown ultraviolet completion with some impact on low energy predictions of Little Higgs models has to be specified. The advantage of these models, relative to supersymmetry, is a much smaller number of free parameters. Excellent reviews can be found in [66, 67].

In Little Higgs models in contrast to the MSSM, new heavy gauge bosons $W_{H}^{ \pm}, Z_{H}$ and $A_{H}$ in the case of the so-called littlest Higgs model without [68] and with T-parity $[69,70]$ are expected. Restricting our discussion to the model with T-parity (LHT), the masses of $W_{H}^{ \pm}$and $Z_{H}$ are typically $\mathscr{O}(700 \mathrm{GeV}) . A_{H}$ is significantly lighter with a mass of a few hundred $\mathrm{GeV}$ and being the lightest particle with odd T-parity can play the role of the dark matter candidate. Concerning the fermion sector, there is a new very heavy $T$-quark necessary to cancel the quadratic divergent contribution of the ordinary top quark to $m_{H}$ and a copy of all SM quarks and leptons is required by T-parity. These mirror quarks and mirror leptons interact with SM particles through the exchange of $W_{H}^{ \pm}$, $Z_{H}$ and $A_{H}$ gauge bosons which in turn implies new flavour and CP-violating contributions to decay amplitudes that are governed by new mixing matrices in the quark and lepton sectors. These matrices can have very different structure than the CKM and PMNS matrices. The mirror quark and leptons can have masses in the range of 500-1500 GeVand could be discovered at the LHC. As we will see in Section 4 their impact on FCNC processes can be sometimes spectacular. Reviews on flavour physics in the LHT model can be found in [71-73].

\section{c) Extra Space Dimensions}

When the number of space-time dimensions is increased, new solutions to the hierarchy prob- 
lems are possible. Most ambitious proposals are models with a warped extra dimension first proposed by Randall and Sandrum (RS) [74] which provide a geometrical explanation of the hierarchy between the Planck scale and the EW scale. Moreover, when the SM fields, except for the Higgs field, are allowed to propagate in the bulk [75-77], these models naturally generate the hierarchies in the fermion masses and mixing angles $[75,77]$ through different localisations of the fermions in the bulk. Yet, this way of explaining the hierarchies in masses and mixings necessarly implies FCNC transitions at the tree level [78-81]. The RS-GIM mechanism [79, 80], combined with an additional custodial protection of flavour violating $Z$ couplings [82-84], allows yet to achieve the agreement with existing data without a considerable fine tuning of parameters. Reviews of [82-84] can be found in [20,62, 85-89]. New theoretical ideas addressing the issue of large FCNC transitions in the RS framework and proposing new protection mechanisms occasionally leading to MFV can be found in [90-95].

In extra dimensional models obvious signatures in high energy processes are the lightest Kaluza-Klein particles, the excited sisters and brothers of the SM particles that can also have significant impact on low energy processes. When KK-parity is present, like in models with universal extra dimensions, then also a dark matter candidate is present. In models with warped extra dimensions and protective custodial symmetries [82, 83, 96-98] imposed to avoid problems with electroweak precision tests (EWPT) and the data on FCNC processes, the gauge group is generally larger than the SM gauge group and similar to the LHT model new heavy gauge bosons are present. However, even in models with custodial symmetries these gauge bosons must be sufficiently heavy $(2-3 \mathrm{TeV})$ in order to be consistent with EWPT. We will denote such RS framework with custodial symmetries by RSc.

As far as the gauge boson sector of the RSc model is concerned, in addition to the SM gauge bosons the lightest new gauge bosons are the KK-gluons, the KK-photon and the electroweak KK gauge bosons $W_{H}^{ \pm}, W^{\prime \pm}, Z_{H}$ and $Z^{\prime}$, all with masses $M_{K K}$ around $2-3 \mathrm{TeV}$. The fermion sector is enriched through heavy KK-fermions (some of them with exotic electric charges) that could in principle be discovered at the LHC. The fermion content of this model is explicitly given in [99], where also a complete set of Feynman rules has been worked out. Detailed analyses of electroweak precision tests and of the parameter $\varepsilon_{K}$ in a RS model without custodial protection can be found in $[100,101]$.

\section{d) Other Models}

There are several other models studied frequently in the literature. These are in particular $Z^{\prime}$ models and models with vector-like heavy quarks [102-104]. Both are present in the RS scenario and I will not discuss them separately. Recently new interest arose in models with a sequential 4th generation which is clearly a possibility. In particular George Hou [105-107] and subsequently Lenz [108], Soni [109] and their collaborators made extensive analyses of FCNC processes in this framework. See also [110]. This NP scenario is quite different from SUSY, the LHT and RS models as the 4th generation of quarks and leptons cannot decouple and if these new fermions exist, they will be found at the LHC. However this direction by itself does not address any hierachy problems and I will not further discuss it in this report. Electroweak precision tests in the presence of fourth generation and other constraints are discussed in [111-114]. 


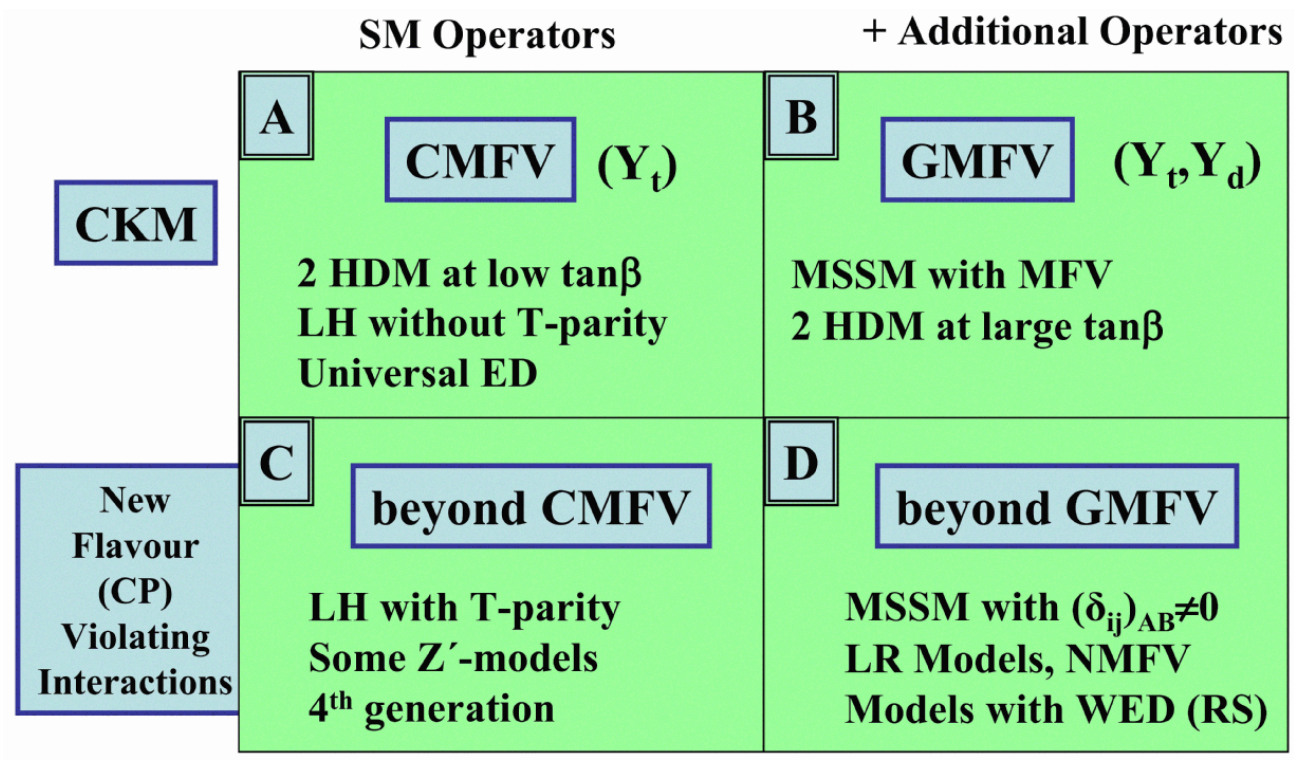

Figure 1: The Flavour Matrix

\subsection{The Flavour Matrix}

The discussion of Section 2.2 suggests to exhibit different extensions of the SM in form of a $2 \times 2$ matrix shown in Fig.1. Let us briefly describe the four entries of this matrix.

The element $(1,1)$ or the class A represents models with CMFV. The SM, the versions of 2HDM's with low $\tan \beta$, the LH model and the ACD model [115] with a universal fifth flat extra dimension belong to this class.

The elements $(1,1)$ and $(1,2)$ or classes $\mathrm{A}$ and $\mathrm{B}$ taken together, the upper row of the flavour matrix, represent the class of models with MFV at large. Basically the new effect in the $(1,2)$ entry relative to $(1,1)$ alone is the appearance of new operators with different Dirac structures that are strongly suppressed in the CMFV framework but can be enhanced if $\tan \beta$ is large or equivalently if $Y_{d}$ cannot be neglected. 2HDM with large $\tan \beta$ belongs to this class. In the past it was believed that the MSSM corresponds to the entry $(1,2)$ only with large $\tan \beta$ but the analysis in [116] has shown that even at low $\tan \beta Y_{d}$ cannot be neglected when the parameter $\mu$ in the Higgs sector is large and gluino contributions become important. We will see below that the presence of new operators, in particular scalar operators, allows to lift the helicity suppression of certain rare decays like $B_{s} \rightarrow \mu^{+} \mu^{-}$, resulting in very different predictions than found in the CMFV models.

The FBMSSM scenario carrying new complex phases that are flavour conserving represents a very special class of MFV models in which the functions $F_{i}$ become complex quantities in contrast to what we stated previously but as these new phases are flavour conserving a natural place for FBMSSM is the upper row of the flavour matrix.

A very interesting class of models is the one represented by the entry $(2,1)$ or the class $\mathrm{C}$. Relative to CMFV it contains new flavour violating interactions, in particular new complex phases, forecasting novel CP-violating effects that may significantly differ from those present in the CMFV class. As there are no new operators relative to the SM ones, no new $B_{i}$-factors and consequently no new non-perturbative uncertainties relative to CMFV models are present. Therefore predictions of models belonging to the $(2,1)$ entry suffer generally from smaller non-perturbative uncertainties 
than models represented by the second column in the flavour matrix in Fig. 1.

When discussing the $(2,1)$ models, it is important to distinguish between models in which new physics couples dominantly to the third generation of quarks, basically the top quark, and models where there is a new sector of fermions that can communicate with the SM fermions with the help of new gauge interactions. Phenomenological approaches with enhanced Z-penguins [117-119], some special $Z^{\prime}$-models [120-122] and the fourth generation models [105, 108-110] belong to the first subclass of $(2,1)$, while the LHT model represents the second subclass.

The entry $(2,2)$ represents the most complicated class of models in which both new flavour violating effects and new operators are relevant. The MSSM with flavour violation coming from the squark sector and RS models are likely to be the most prominent members of this class of models. The NMFV approach of [123] and left-right symmetric models belong also to this class. The spurion technology for this class of models has been developed by Feldmann and Mannel [39].

\subsection{The Little Hierarchy Problem}

As we have seen, the stabilization of the Higgs mass under radiative corrections requires NP at scales $\mathscr{O}(1 \mathrm{TeV})$. Yet EWPT performed first at LEP/SLC and subsequently extended at Tevatron imply that NP, unless properly screened, can only appear at scales of 5-10 $\mathrm{TeV}$ or higher. The situation is much worse in FCNC processes. There the masses of new particles carrying flavour and having $\mathscr{O}(1)$ couplings cannot contribute at tree level unless their masses are larger than $1000 \mathrm{TeV}$ or even more. A detailed analysis of this issue can be found in particular in [124].

Thus in order to keep the solutions to the hierarchy problems discussed above alive, protective symmetries must be present in order to suppress NP effects to electroweak precision observables (EWPO) and to FCNC processes in spite of NP being present at scales $\mathscr{O}(1 \mathrm{TeV})$ or lower. In this context the custodial SU(2) symmetry in the case of EWPT should be mentioned. In the framework of the LHT model this symmetry is guarantied by T-parity. For the FCNC processes we need generally a GIM mechanism which forbids tree level contributions. If this mechanism is violated and FCNC transitions occur already at tree level other protections are necessary. In RS models the so-called RS-GIM mechanism $[79,80]$ and the recently pointed out custodial protection for flavour violating $Z$ couplings [82-84] play an important role.

In this context MFV is very popular as models with MFV can naturally satisfy the existing FCNC constraints. While this framework will play a role below, we will in Section 4 dominantly present the results coming from the non-MFV scenarios discussed in Section 2.4.

\section{20 Goals in Flavour Physics for the Next Decade}

We will now list 20 goals in flavour physics for the coming decade. The order in which these goals will be listed does not represent by any means a ranking in importance. In this section each goal will be summarized very briefly including some references where further details can be found. In Section 4 we will concentrate on the goals 1, 3, 4, 6 and 10 which most likely will play the central role in quark flavour physics in the coming years. We will close Section 4 by correlating these goals with the goals 16, 17 and 18 that deal with lepton physics in the context of supersymmetric flavour models. Let us now list the 20 goals in question. 


\section{Goal 1: The CKM Matrix from tree level decays}

This determination would give us the values of the elements of the CKM matrix without NP pollution. From the present perspective most important are the determinations of $\left|V_{u b}\right|$ and $\gamma$ because they are presently not as well known as $\left|V_{c b}\right|$ and $\left|V_{u s}\right|$. However, a precise determination of $\left|V_{c b}\right|$ is also important as $\varepsilon_{K}, B r\left(K^{+} \rightarrow \pi^{+} v \bar{v}\right)$ and $B r\left(K_{L} \rightarrow \pi^{0} v \bar{v}\right)$ are roughly proportional to $\left|V_{c b}\right|^{4}$. While Super-B facilities accompanied by improved theory should be able to determine $\left|V_{u b}\right|$ and $\left|V_{c b}\right|$ with precision of $1-2 \%$, the best determination of the angle $\gamma$ in the first half of the next decade will come from LHCb. An error of a few degrees should be achievable around 2015 and this measurement could also be improved at Super-B machines.

\section{Goal 2: Improved Lattice Calculations of Hadronic Parameters}

The knowledge of the meson decay constants $F_{B_{s}}, F_{B_{d}}$ and of various $B_{i}$ parameters with high precision would allow in conjunction with Goal 1 to make precise calculations of $\Delta M_{s}, \Delta M_{d}, \varepsilon_{K}$, $\operatorname{Br}\left(B_{s, d} \rightarrow \mu^{+} \mu^{-}\right)$and of other observables in the SM. We could then directly see whether the SM is capable of describing these observables or not. The most recent unquenched calculations allow for optimism and in fact a very significant progress in the calculation of $\hat{B}_{K}$ has been made recently. We will discuss its implications in Section 4.

For completeness we collect here some selected non-perturbative parameters relevant for FCNC processes. The present lattice values, that are relevant for $B_{s, d}^{0}-\bar{B}_{s, d}^{0}$ mixings, taken from [125] read

$$
F_{B_{s}} \sqrt{\hat{B}_{B_{s}}}=270(30) \mathrm{MeV}, \quad F_{B_{d}} \sqrt{\hat{B}_{B_{d}}}=225(25) \mathrm{MeV},
$$

while the HPQCD collaboration [126] finds similar values but smaller errors,

$$
F_{B_{s}} \sqrt{\hat{B}_{B_{s}}}=266(18) \mathrm{MeV}, \quad F_{B_{d}} \sqrt{\hat{B}_{B_{d}}}=216(15) \mathrm{MeV} .
$$

Other values that should be improved are the $\hat{B}_{i}$ parameters themselves that will play some role in predicting the branching ratios for $B_{s, d} \rightarrow \mu^{+} \mu^{-}$as we proceed. The present lattice results read [125]

$$
\frac{\hat{B}_{s}}{\hat{B}_{d}}=1.00 \pm 0.03, \quad \hat{B}_{d}=1.22 \pm 0.12, \quad \hat{B}_{s}=1.22 \pm 0.12 .
$$

Also the accuracy of the $B_{i}$ parameters related to new operators present in the classes $B$ and $D$ in the flavour matrix should be improved.

In this context one should mention the determination of quark masses and of the QCD coupling constant $\alpha_{s}\left(M_{Z}\right)$ that should still be improved in order to reduce the parametric uncertainties in the predictions for various branching ratios. Here important advances have been made recently. Let me just quote [127]

$$
m_{b}\left(m_{b}\right)=(4.163 \pm 0.016) \mathrm{GeV}, \quad m_{c}\left(m_{c}\right)=1.279 \pm 0.013 \mathrm{GeV},
$$

with the latter very relevant for the decay $K^{+} \rightarrow \pi^{+} v \bar{v}$. Similarly,

$$
m_{s}(2 \mathrm{GeV})=(91 \pm 7) \mathrm{MeV}, \quad m_{t}\left(m_{t}\right)=163.5 \pm 1.7 \mathrm{GeV},
$$

with the value of $m_{s}(2 \mathrm{GeV})$ given recently by Leutwyler [128]. This agrees very well with [129], where $94 \pm 6 \mathrm{MeV}$ has been quoted. 
Finally, two impressive determinations of $\alpha_{s}\left(M_{Z}\right)$ should be mentioned here. One is from hadronic $\mathrm{Z}$ and $\tau$ decays [130] resulting in $\alpha\left(M_{Z}\right)=0.1198 \pm 0.0015$ and the second from the $\tau$ hadronic width [131] with the result $\alpha\left(M_{Z}\right)=0.1180 \pm 0.0008$. The latest world average reads [132]

$$
\alpha\left(M_{Z}\right)=0.1184 \pm 0.0007
$$

\section{Goal 3: Is $\varepsilon_{\mathrm{K}}$ consistent with $S_{\psi \mathrm{K}_{\mathrm{S}}}$ within the $\mathrm{SM}$ ?}

The recent improved value of $\hat{B}_{K}$ from unquenched lattice QCD acompanied by a more careful look at $\varepsilon_{K}$ suggest that the size of CP violation measured in $B_{d} \rightarrow \psi K_{S}$ might be insufficient to describe $\varepsilon_{K}$ within the SM. Clarification of this new tension is important as the $\sin 2 \beta-\varepsilon_{K}$ correlation in the SM is presently the only relation between CP violation in the $B_{d}$ and $K$ systems that can be tested experimentally. We will return to this issue in Section 4.

\section{Goal 4: Is $\mathbf{S}_{\psi \phi}$ much larger than its tiny $\mathbf{S M}$ value?}

Within the SM CP violation in the $B_{s}$ system is predicted to be very small. The best known representation of this fact is the value of the mixing induced $\mathrm{CP}$ asymmetry: $\left(S_{\psi \phi}\right)_{\mathrm{SM}} \approx 0.04$. The present data from $\mathrm{CDF}$ and D0 indicate that $\mathrm{CP}$ violation in the $B_{s}$ system could be much larger, $S_{\psi \phi}=0.81_{-0.32}^{+0.12}$ [133]. This is a very interesting deviation from the SM. Its clarification is of utmost importance and I will return to this question in Section 4. Fortunately, we should know the answer to this question within the coming years as CDF, D0, LHCb, ATLAS and CMS will make big efforts to measure $S_{\psi \phi}$ precisely.

\section{Goal 5: Non-Leptonic Two-Body B Decays and Related Puzzles}

The best information on $\mathrm{CP}$ violation in the $B$ system to date comes from two-body nonleptonic decays of $B_{d}$ and $B^{ \pm}$mesons. While until now these decays dominated this field, $\mathrm{LHCb}$ will extend these studies in an important manner to $B_{s}$ and $B_{c}$ decays. This is clearly a challenging field not only for experimentalist but in particular also for theorists due to potential hadronic uncertainties. Yet, in the last ten years an impressive progress has been made in measuring many channels, in particular $B \rightarrow \pi \pi$ and $B \rightarrow \pi K$ decays, and in developing a number of methods like QCD factorization [134, 135], the Perturbative QCD approach [136], SCET [137-141] and more phenomenological approaches based on flavour symmetries [119,142]. Excellent reviews of this subject have been given by Buchalla [143], Fleischer [144] and Silvestrini [145]. They contain a lot of useful material. I think this field will continue to be important for the tests of the CKM framework in view of very many channels whose branching ratios should be measured in the next decade with a high precision. This is also a place where the structure of QCD effects in the interplay with weak interactions can be studied very well and the combination of the lessons gained from this field with those coming from theoretically cleaner decays discussed subsequently will undoubtly enrich our view on flavour physics.

On the other hand in view of potential hadronic uncertainties present in the branching ratios and direct $\mathrm{CP}$ asymmetries these observables in my opinion will not provide definite answers about NP if the latter contributes to them only at the level of $20 \%$ or less. On the other hand mixing induced CP-asymmetries like $S_{\psi K_{S}}, S_{\psi \phi}$ and alike being theoretically much cleaner will continue to be very important for the tests of NP. Let me then just briefly discuss a number of departures from the SM predictions which await resolution in the coming years. 
First of all the angle $\beta$ has been measured in several other decays, in particular in penguin dominated decays like $B \rightarrow \phi K_{S}$ or $B \rightarrow \eta^{\prime} K_{S}$ with the result that it is generally smaller than $(\sin 2 \beta)_{\psi K_{S}}$, putting the SM and MFV in some difficulties. Clarification of this disagreement is an important goal for the next decade. While this tension became weaker with time, the theoretically clean asymmetry $S_{\phi K_{S}}$ still remains to be significantly smaller than the expected value of approximately 0.67 [133]:

$$
S_{\phi K_{S}}=0.44 \pm 0.17
$$

This tension cannot be resolved at $\mathrm{LHCb}$ and its resolution will remain as one of the important goals for Super Belle at KEK and later the Super-B machine in Frascati, although an insight on a possible anomalous behaviour in this asymmetry should be gained at LHCb through the study of CP violation in $B_{s} \rightarrow \phi \phi$ [146].

We will see in Section 4 that the desire to explain the value in (3.7) in the framework of some supersymetric models will have interesting implications for other CP-violating observables like the direct CP asymmetry in $B \rightarrow X_{s} \gamma$ and electric dipole moments.

Next the rather large difference in the direct CP asymmetries $A_{C P}\left(B^{-} \rightarrow K^{-} \pi^{0}\right)$ and $A_{C P}\left(\bar{B}^{0} \rightarrow\right.$ $K^{-} \pi^{+}$) observed by the Belle and BaBar collaborations has not been expected but it could be due to our insufficient understanding of hadronic effects rather than NP. Similar comments apply to certain puzzles in $B \rightarrow \pi K$ decays [119] which represent additional tensions that decreased with time but did not fully disappear [147]. For a different view see [148].

Finally of particular interest is the mixing induced CP-asymmetry in $B \rightarrow \pi^{0} K_{S}$ which appears to indicate still some tensions with the SM expectations $[119,149,150]$ although this is inconclusive at present. For the most recent analysis see [148].

Goal 6: $\operatorname{Br}\left(\mathbf{B}_{\mathrm{s}, \mathbf{d}} \rightarrow \mu^{+} \mu^{-}\right)$

In the SM and in several of its extentions $\operatorname{Br}\left(B_{s} \rightarrow \mu^{+} \mu^{-}\right)$is found in the ballpark of $3-$ $5 \cdot 10^{-9}$, which is by an order of magnitude lower than the present bounds from CDF and D0. A discovery of $\operatorname{Br}\left(B_{s, d} \rightarrow \mu^{+} \mu^{-}\right)$at $\mathscr{O}\left(10^{-8}\right)$ would be a clear signal of NP, possibly related to Higgs penguins. LHCb can reach the SM level for this branching ratio in the first years of its operation. From my point of view, similar to $S_{\psi \phi}$, precise measurements of $\operatorname{Br}\left(B_{s} \rightarrow \mu^{+} \mu^{-}\right)$and later of a more suppressed branching ratio $\operatorname{Br}\left(B_{d} \rightarrow \mu^{+} \mu^{-}\right)$are among the most important goals in flavour physics in the coming years. We will discuss both decays in Section 4.

Goal 7: $\mathbf{B} \rightarrow \mathbf{X}_{\mathbf{s}, \mathbf{d}} \gamma, \mathbf{B} \rightarrow \mathbf{K}^{*}(\rho) \gamma$ and $\mathbf{A}_{\mathrm{CP}}^{\operatorname{dir}}(\mathbf{b} \rightarrow \mathbf{s} \gamma)$

The radiative decays in question, in particular $B \rightarrow X_{s} \gamma$, played an important role in constraining NP in the last 15 years because both the experimental data and also the theory have been already in a good shape for some time with the NNLO calculations of $\operatorname{Br}\left(B \rightarrow X_{s} \gamma\right)$ being at the forefront of perturbative QCD calculations in weak decays. Both theory and experiment reached roughly $10 \%$ precision and the agreement of the SM with the data is good implying not much room left for NP contributions. Still further progress both in theory and experiment should be made to further constrain NP models. This will only be possible when Super-B machines enter their operation. Of particular interest is the direct CP asymmetry $A_{\mathrm{CP}}^{\mathrm{dir}}(b \rightarrow s \gamma)$ that is similar to $S_{\psi \phi}$ predicted to be tiny $(0.5 \%)$ in the SM but could be much larger in some of its extensions as discussed in Section 4. 


\section{Goal 8: $\mathbf{B} \rightarrow \mathbf{X}_{\mathbf{s}} \mathbf{l}^{+} \mathbf{I}^{-}$and $\mathbf{B} \rightarrow \mathbf{K}^{*} \mathbf{l}^{+} \mathbf{I}^{-}$}

While the branching ratios for $B \rightarrow X_{s} l^{+} l^{-}$and $B \rightarrow K^{*} l^{+} l^{-}$put already significant constraints on NP, the angular observables, CP-conserving ones like the well known forward-backward asymmetry and CP-violating ones will definitely be very useful for distinguishing various extensions of the SM. Recently, a number of detailed analyses of various $\mathrm{CP}$ averaged symmetries and $\mathrm{CP}$ asymmetries provided by the angular distributions in the exclusive decay $B \rightarrow K^{*}(\rightarrow K \pi) l^{+} l^{-}$ have been performed in [151-153]. In particular the zeroes of some of these observables can be accurately predicted. Belle and BaBar provided already interesting results for the best known forward-backward asymmetry but the data have to be improved in order to see whether some sign of NP is seen in this asymmetry. Future studies by the LHCb and Super-B machines will be able to contribute here in a significant manner.

Goal 9: $\mathbf{B}^{+} \rightarrow \tau^{+} v$ and $\mathbf{B}^{+} \rightarrow \mathbf{D}^{\mathbf{0}} \tau^{+} v$

The SM expression for the branching ratio of the tree-level decay $B^{+} \rightarrow \tau^{+} v$ is given by

$$
\operatorname{Br}\left(B^{+} \rightarrow \tau^{+} v\right)_{\mathrm{SM}}=\frac{G_{F}^{2} m_{B^{+}} m_{\tau}^{2}}{8 \pi}\left(1-\frac{m_{\tau}^{2}}{m_{B^{+}}^{2}}\right)^{2} F_{B^{+}}^{2}\left|V_{u b}\right|^{2} \tau_{B^{+}} .
$$

In view of the parametric uncertainties induced in (3.8) by $F_{B^{+}}$and $V_{u b}$, in order to find the SM prediction for this branching ratio one can rewrite it as follows [55]:

$$
B r\left(B^{+} \rightarrow \tau^{+} v\right)_{\mathrm{SM}}=\frac{3 \pi}{4 \eta_{B} S_{0}\left(x_{t}\right) \hat{B}_{B_{d}}} \frac{m_{\tau}^{2}}{M_{W}^{2}}\left(1-\frac{m_{\tau}^{2}}{m_{B^{+}}^{2}}\right)^{2}\left|\frac{V_{u b}}{V_{t d}}\right|^{2} \tau_{B^{+}} \Delta M_{d} .
$$

Here $\Delta M_{d}$ is supposed to be taken from experiment and

$$
\left|\frac{V_{u b}}{V_{t d}}\right|^{2}=\left(\frac{1}{1-\lambda^{2} / 2}\right)^{2} \frac{1+R_{t}^{2}-2 R_{t} \cos \beta}{R_{t}^{2}},
$$

with $R_{t}$ and $\beta$ determined by means of (4.5) in Section 4. In writing (3.9), we used $F_{B} \simeq F_{B^{+}}$and $m_{B_{d}} \simeq m_{B^{+}}$. We then find [55]

$$
\operatorname{Br}\left(B^{+} \rightarrow \tau^{+} v\right)_{\mathrm{SM}}=(0.80 \pm 0.12) \times 10^{-4} .
$$

This result agrees well with a recent result presented by the UTfit collaboration [154].

On the other hand, the present experimental world avarage based on results by BaBar $[155$, $156]$ and Belle $[157,158]$ reads $[159]$

$$
\operatorname{Br}\left(B^{+} \rightarrow \tau^{+} v\right)_{\exp }=(1.73 \pm 0.35) \times 10^{-4},
$$

which is roughly by a factor of 2 higher than the SM value. We can talk about a tension at the $2.5 \sigma$ level.

While the final data from $\mathrm{BaBar}$ and Belle will lower the exparimental error on $\operatorname{Br}\left(B^{+} \rightarrow \tau^{+} v\right)$, the full clarification of a possible discrepancy between the SM and the data will have to wait for the data from Super-B machines. Also improved values for $F_{B}$ from lattice and $\left|V_{u b}\right|$ from tree level decays will be important if some NP like charged Higgs is at work here. The decay $B^{+} \rightarrow$ $D^{0} \tau^{+} v$ being sensitive to different couplings of $H^{ \pm}$can contribute significantly to this discussion 
but formfactor uncertainties make this decay less theoretically clean. A thorough analysis of this decay is presented in [160] where further references can be found.

Interestingly, the tension between theory and experiment in the case of $\operatorname{Br}\left(B^{+} \rightarrow \tau^{+} v\right)$ increases in the presence of a tree level $H^{ \pm}$exchange which interferes destructively with the $W^{ \pm}$ contribution. As addressed long time ago by Hou [161] and in modern times calculated first by Akeroyd and Recksiegel [162], and later by Isidori and Paradisi [163], one has in the MSSM with MFV and large $\tan \beta$

$$
\frac{B r\left(B^{+} \rightarrow \tau^{+} v\right)_{\mathrm{MSSM}}}{B r\left(B^{+} \rightarrow \tau^{+} v\right)_{\mathrm{SM}}}=\left[1-\frac{m_{B}^{2}}{m_{H^{ \pm}}^{2}} \frac{\tan ^{2} \beta}{1+\varepsilon \tan \beta}\right]^{2},
$$

with $\varepsilon$ collecting the dependence on supersymmetric parameters. This means that in the MSSM this decay can be strongly suppressed unless the choice of model parameters is such that the second term in the parenthesis is larger than 2. Such a possibility that would necessarily imply a light charged Higgs and large $\tan \beta$ values seems to be very unlikely in view of the constraints from other observables [164]. Recent summaries of $H^{ \pm}$physics can be found in [165,166].

\section{Goal 10: Rare Kaon Decays}

Among the top highlights of flavour physics in the next decade will be the measurements of the branching ratios of two golden modes $K^{+} \rightarrow \pi^{+} v \bar{v}$ and $K_{L} \rightarrow \pi^{0} v \bar{v} . K^{+} \rightarrow \pi^{+} v \bar{v}$ is CP conserving while $K_{L} \rightarrow \pi^{0} v \bar{v}$ is governed by CP violation. Both decays are dominated in the SM and many of its extensions by $Z$ penguin contributions. It is well known that these decays are theoretically very clean and are known in the SM including NNLO QCD corrections and electroweak corrections [34-36]. Moreover, extensive calculations of isospin breaking effects and non-perturbative effects have been done [167,168]. The present theoretical uncertainties in $\operatorname{Br}\left(K^{+} \rightarrow \pi^{+} v \bar{v}\right)$ and $\operatorname{Br}\left(K_{L} \rightarrow\right.$ $\left.\pi^{0} v \bar{v}\right)$ are at the level of $2-3 \%$ and $1-2 \%$, respectively.

We will discuss these decays in more detail in Section 4 but let me stress already here that the measurements of their branching ratios with an accuraccy of $10 \%$ will give us a very important insight into the physics at short distance scales. NA62 at CERN in the case of $K^{+} \rightarrow \pi^{+} v \bar{v}$ and KOTO at J-PARC in the case of $K_{L} \rightarrow \pi^{0} v \bar{v}$ will tell us how these two decays are affected by NP.

The decays $K_{L} \rightarrow \pi^{0} l^{+} l^{-}$are not as theoretically clean as the $K \rightarrow \pi v \bar{v}$ chanels and are less sensitive to NP contributions but they probe different operators beyond the SM and having accurate branching ratios for them would certainly be useful. Further details on this decay can be found in [169-174].

Goal 11: Rare B Decays $\mathbf{B} \rightarrow \mathbf{X}_{\mathbf{s}} v \bar{v}, \mathbf{B} \rightarrow \mathbf{K}^{*} v \bar{v}$ and $\mathbf{B} \rightarrow \mathbf{K} v \bar{v}$

Also $B$ decays with $v \bar{v}$ in the final state provide a very good test of modified $Z$ penguin contributions $[175,176]$, but their measurements appear to be even harder than those of the rare $K$ decays just discussed. Recent analyses of these decays within the SM and several NP scenarios can be found in $[177,178]$.

The inclusive decay $B \rightarrow X_{s} v \bar{v}$ is theoretically as clean as $K \rightarrow \pi \nu \bar{v}$ decays but the parametric uncertainties are a bit larger. The two exclusive channels are affected by formfactor uncertainties but recently in the case of $B \rightarrow K^{*} v \bar{v}$ [177] and $B \rightarrow K v \bar{v}$ [178] significant progress has been made. In the latter paper this has been achieved by considering simultaneously also $B \rightarrow K l^{+} l^{-}$. Very recently non-perturbative tree level contributions from $B^{+} \rightarrow \tau^{+} v$ to $B^{+} \rightarrow K^{+} v \bar{v}$ and $B^{+} \rightarrow$ $K^{*+} v \bar{v}$ at the level of roughly $10 \%$ have been pointed out [179]. 
The interesting feature of these three $b \rightarrow s v \bar{v}$ transitions, in particular when taken together, is their sensitivity to right-handed currents [177]. Super-B machines should be able to measure them at a satisfactory level.

\section{Goal 12: Lattice Calculations of Hadronic Matrix Elements in $\varepsilon^{\prime} / \varepsilon$}

One of the important actors of the previous decade in flavour physics was the ratio $\varepsilon^{\prime} / \varepsilon$ that measures the size of the direct $\mathrm{CP}$ violation in $K_{L} \rightarrow \pi \pi$ relative to the indirect $\mathrm{CP}$ violation described by $\varepsilon_{K}$. In the SM $\varepsilon^{\prime}$ is governed by QCD penguins but receives also an important distructively interfering contribution from electroweak penguins that is generally much more sensitive to NP than the QCD penguin contribution.

Here the problem is the strong cancellation of QCD penguin contributions and electroweak penguin contributions to $\varepsilon^{\prime} / \varepsilon$ and in order to obtain useful predictions the precision on the corresponding hadronic parameters $B_{6}$ and $B_{8}$ should be at least $10 \%$. Lattice theorists around Norman Christ hope to make progress on $B_{6}, B_{8}$ and other $\varepsilon^{\prime} / \varepsilon$ related hadronic matrix elements in the coming decade. This would really be good, as the calculations of short distance contributions to this ratio (Wilson coefficients of QCD and electroweak penguin operators) have been known already for 16 years at the NLO level $[180,181]$ and present technology could extend them to the NNLO level if necessary.

The present experimental world average from NA48 [182] and KTeV [183,184],

$$
\varepsilon^{\prime} / \varepsilon=(16.8 \pm 1.4) \cdot 10^{-4}
$$

could have an important impact on several extentions of the SM discussed in Section 4 if $B_{6}$ and $B_{8}$ were known. An analysis of $\varepsilon^{\prime} / \varepsilon$ in the LHT model demonstrates this problem in explicit terms [185]. If one uses $B_{6}=B_{8}=1$ as obtained in the large $\mathrm{N}$ approach, $\left(\varepsilon^{\prime} / \varepsilon\right)_{\mathrm{SM}}$ is in the ballpark of the experimental data and sizable departures of $\operatorname{Br}\left(K_{L} \rightarrow \pi^{0} v \bar{v}\right)$ from its SM value are not allowed. $K^{+} \rightarrow \pi^{+} v \bar{v}$ being CP conserving and consequently not as strongly correlated with $\varepsilon^{\prime} / \varepsilon$ as $K_{L} \rightarrow \pi^{0} v \bar{v}$ could still be enhanced by $50 \%$. On the other hand if $B_{6}$ and $B_{8}$ are different from unity and $\left(\varepsilon^{\prime} / \varepsilon\right)_{\mathrm{SM}}$ disagrees with experiment, much more room for enhancements of rare $\mathrm{K}$ decay branching ratios through NP contributions is available. Reviews of $\varepsilon^{\prime} / \varepsilon$ can be found in [186, 187].

Goal 13: CP Violation in Charm Decays, $\mathbf{D}^{+}\left(\mathbf{D}_{\mathrm{s}}^{+}\right) \rightarrow \mathbf{I}^{+} v$ and $\mathbf{D}^{0} \rightarrow \mu^{+} \mu^{-}$

Charm physics has been for many years shadowed by the successes of $K$ decays and $B$ decays, although a number of experimental groups and selected theorists have made a considerable effort to study them. This is due to the GIM mechanism being very effective in suppressing the FCNC transitions in this sector, long distance contributions plaguing the evaluation of $\Delta M_{D}$ and insensitivity to top physics in the loops. However, the large $D^{0}-\bar{D}^{0}$ mixing discovered in 2007 [188-190] and good prospects for the study of CP violation in the above decays at Super Belle and Super $B$ in Frascati gave a new impetus to this field. The main targets here are:

- Dedicated studies of CP Violation in $D$ decays that is predicted to be very small in the SM, but could be strongly enhanced beyond the SM and is theoretically much cleaner than $\Delta M_{D}$,

- Dedicated studies of $D^{+} \rightarrow \mu^{+} v_{\mu}, D^{+} \rightarrow \tau^{+} v_{\tau}$ and $D_{s} \rightarrow \tau^{+} v_{\tau}$ with higher experimental and lattice accuracy with the aim to study charged Higgs effects, 
- Rare decays $D^{0} \rightarrow \mu^{+} \mu^{-}$and $D_{s} \rightarrow \mu^{+} \mu^{-}$.

Excellent reviews can be found in [191, 192]. Various aspects of charm physics are discussed in [193-200].

The first possible sign of NP appeared in $D_{s}^{+} \rightarrow l^{+} v$ decays some time ago and in 2008 a $3 \sigma$ discrepancy between the SM and the data has been declared. Meanwhile this tension decreased to $2 \sigma$ and in order to have a clear picture we have to wait for the new data with higher statistics and further improved lattice calculations of the relevant $D$ weak decay constants even if the latter are already rather precise [201]. In fact this example shows how much fun we will have to compare the data with theory when both experiments and lattice calculations improve.

\section{Goal 14: CP Violation in the Lepton Sector and $\theta_{13}$}

The mixing angles $\theta_{12}$ and $\theta_{23}$ are already known with respectable precision. The obvious next targets in this field are $\theta_{13}$ and the CP phase $\delta_{\text {PMNS }}$. Clearly the discovery of CP violation in the lepton sector would be a very important mile stone in particle physics for many reasons. In particular the most efficient explanations of the BAU these days follow from leptogenesis. While in the past the necessary size of $\mathrm{CP}$ violation was obtained from new sources of $\mathrm{CP}$ violation at very high see-saw scales, the inclusion of flavour effects, in particular in resonant leptogenesis, gave hopes for the explanation of the BAU using only the phases in the PMNS matrix. This implies certain conditions for the parameters of this matrix, that is the relevant $\delta_{\mathrm{PMNS}}$, two Majorana phases and $\theta_{13}$. As there was a separate talk on neutrino physics at this conference let me just refer to this talk and the review in [202] for the relevant references. A recent review of models for neutrino masses is given in [203].

\section{Goal 15: Tests of $\mu-\mathbf{e}$ and $\mu-\tau$ Universalties}

Lepton flavour violation (LFV) and the related breakdown of universality can be tested in meson decays by studying the ratios [204,205]

$$
R_{\mu e}=\frac{B r\left(K^{+} \rightarrow \mu^{+} v\right)}{B r\left(K^{+} \rightarrow e^{+} v\right)}, \quad R_{\mu \tau}=\frac{B r\left(B^{+} \rightarrow \mu^{+} v\right)}{B r\left(B^{+} \rightarrow \tau^{+} v\right)},
$$

where the sum over different neutrino flavours is understood. The first case is a high precision affair both for experimentalists and theorists as both groups decreased the uncertainties in $R_{\mu e}$ well below $1 \%$ with a precision of $0.5 \%$ recently achieved at CERN. It will continue to constitute an important test of the $\mu-e$ universality. The ratio $R_{\mu \tau}$ is even more sensitive to NP contributions but it will still take some time before it will be known with good precision.

\section{Goal: 16 Flavour Violation in Charged Lepton Decays}

The search for LFV clearly belongs to the most important goals in flavour physics. The nonvanishing neutrino masses and neutrino oscillations as well as the see-saw mechanism for the generation of neutrino masses have given an impressive impetus to the study of flavour violation in the lepton sector in the last ten years. In the SM with right-handed Dirac neutrinos, the smallness of neutrino masses implies tiny branching ratios for LFV processes. For instance

$$
\operatorname{Br}(\mu \rightarrow e \gamma)_{\mathrm{SM}} \approx 10^{-54},
$$

which is more than 40 orders of magnitude below the $90 \%$ C.L. upper bound from the MEGA Collaboration [206]

$$
\operatorname{Br}(\mu \rightarrow e \gamma)<1.2 \cdot 10^{-11}
$$


Therefore any observation of LFV would be a clear sign of NP. While we hope that new flavoured leptons will be observed at the LHC, even if this will not turn out to be the case, LFV has the following virtue: sensitivity to short distance scales as high as $10^{10}-10^{14} \mathrm{GeV}$, in particular when the see-saw mechanism is at work.

The prospects for the measurements of LFV processes with much higher sensitivity than presently available in the next decade look very good. In particular the MEG experiment at PSI [207] should be able to test $\operatorname{Br}(\mu \rightarrow e \gamma)$ at the level of $\mathscr{O}\left(10^{-13}-10^{-14}\right)$, and the Super Flavour Factory [10] is planned to reach a sensitivity for $\operatorname{Br}(\tau \rightarrow \mu \gamma)$ of at least $\mathscr{O}\left(10^{-9}\right)$. The planned accuracy of SuperKEKB of $\mathscr{O}\left(10^{-8}\right)$ for $\tau \rightarrow \mu \gamma$ is also of great interest. Very important will also be an improved upper bound on $\mu-e$ conversion in Ti. In this context the dedicated J-PARC experiment PRISM/PRIME [208] should reach the sensitivity of $\mathscr{O}\left(10^{-18}\right)$. This means an improvement by six orders of magnitude relative to the present upper bound from SINDRUM II at PSI [209].

Now the various supersymmetric models, the LHT model and the RS models are capable of reaching the bound in (3.17) and in fact this bound puts already rather stringent constraints on the parameters of these models. For instance in the case of the LHT model the mixing matrix in the mirror lepton sector has to be either very hierarchical, at least as hierarchical as the CKM matrix or the mirror-lepton spectrum has to be quasi-degenerate [73,210,211]. Analogous constraints exist in other models. We will discuss some aspects of LFV in Section 4.

In order to distinguish various NP scenarios that come close to the bound in (3.17) it will be essential to study a large set of decays to three leptons in the final state. Indeed, while in the MSSM [212-216] the dominant role in the decays with three leptons in the final state and in $\mu-e$ conversion in nuclei is played by the dipole operator, in $[210,211]$ it was found that this operator is much less relevant in the LHT model, with $Z^{0}$ penguin and box diagrams being the dominant contributions. This implies a striking difference between various ratios of branching ratios of type $\operatorname{Br}\left(l_{i} \rightarrow 3 l_{j}\right) / \operatorname{Br}\left(l_{i} \rightarrow l_{j} \gamma\right)$ in the MSSM, where they are typically $\mathscr{O}\left(10^{-2}-10^{-3}\right)$ and in the LHT model, where they are $\mathscr{O}\left(10^{-1}\right)$ [73]. A very recent short review of these topics can be found in [217].

There exist also interesting correlations between leptogenesis and LFV but this is beyond the scope of this presentation. Additional correlations relevant for LFV will be discussed in Section 4 .

\section{Goal 17: Electric Dipole Moments}

$\mathrm{CP}$ violation has only been observed in flavour violating processes. Non-vanishing electric dipole moments signal $\mathrm{CP}$ violation in flavour conserving transitions. In the SM CP violation in flavour conserving processes is very strongly suppressed as best expressed by the SM values of electric dipole moments of the neutron and electron that amount to [218]

$$
d_{n} \approx 10^{-32} \mathrm{e} \mathrm{cm}, \quad d_{e} \approx 10^{-38} \mathrm{e} \mathrm{cm} .
$$

This should be compared with the present experimental bounds [219,220]

$$
d_{n} \leq 2.9 \cdot 10^{-26} \mathrm{e} \mathrm{cm} . \quad d_{e} \leq 1.6 \cdot 10^{-27} \mathrm{e} \mathrm{cm} .
$$

They should be improved in the coming years by 1-2 orders of magnitude.

Similar to LFV, an observation of a non-vanishing EDM would imply necessarily NP at work. Consequently correlations between LFV and EDM's in specific NP scenarios are to be expected, 
in particular in supersymmetric models, as both classes of observables are governed by dipole operators. A rather complete analysis of such correlations has been recently presented in [221] where further references can be found. We will encounter some specific examples in Section 4.

Goal 18: Clarification of the $(\mathrm{g}-2)_{\mu}$ Anomaly

The measured anomalous magnetic moment of the electron, $(g-2)_{e}$, is in an excellent agreement with SM expectations. On the other hand, the measured anomalous magnetic moment of the muon, $(g-2)_{\mu}$, is significantly larger than its SM value. The most recent SM prediction reads [222]

$$
a_{\mu}^{\mathrm{SM}}=116591834(49) \cdot 10^{-11}
$$

and the experimental value from BNL $[223,224]$

$$
a_{\mu}^{\exp }=116592080(63) \cdot 10^{-11},
$$

where $a_{\mu}=(g-2)_{\mu} / 2$. Consequently,

$$
\Delta a_{\mu}=a_{\mu}^{\exp }-a_{\mu}^{\mathrm{SM}}=(2.5 \pm 0.8) \times 10^{-9},
$$

implying a $3.1 \sigma$ deviation from the SM value. Similar results can be found in [225, 226].

Hadronic contributions to $(g-2)_{\mu}$ make the comparison of data and theory a bit problematic. Yet, as this anomaly has been with us already for a decade and tremendous effort by a number of theorists has been made to clarify this issue, this anomaly could indeed come from NP.

The MSSM with large $\tan \beta$ and sleptons with masses below $400 \mathrm{GeV}$ is capable of reproducing the experimental value of $a_{\mu}$ provided the $\mu$ parameter in the Higgs Lagrangian has a specific sign, positive in my conventions:

$$
\frac{a_{\mu}^{\mathrm{MSSM}}}{1 \times 10^{-9}} \approx 1.5\left(\frac{\tan \beta}{10}\right)\left(\frac{300 \mathrm{GeV}}{m_{\tilde{\ell}}}\right)^{2} \operatorname{sgn} \mu .
$$

Moreover an interesting correlation between the amount of necessary shift $\Delta a_{\mu}$ and the value of $\operatorname{Br}(\tau \rightarrow \mu \gamma)$ and $\operatorname{Br}(\mu \rightarrow e \gamma)$ exists [227], implying that these two branching ratios could be as high as $4 \cdot 10^{-9}$ and $3 \cdot 10^{-12}$, respectively and thus in the reach of dedicated experiments in the coming years. Other correlations of this type in supersymmetric flavour models will be discussed in Section 4. On the other hand the LHT fails to reproduce the data in (3.21) and $a_{\mu}$ in this model is within the uncertainties indistiguishable from its SM value [210,228]. Apparently there is no visible correlation between NP in $a_{\mu}$ and LFV in this model. Thus if the data in (3.21) remain, they would favour the MSSM over the LHT. Recent reviews on $(g-2)_{\mu}$ can be found in [222,229-232].

\section{Goal 19: Flavour Violation at High Energy}

Our presentation deals mainly with tests of flavour and CP violation in low energy processes. However, at the LHC it will be possible to investigate these phenomena also in high energy processes, in particular in top quark decays. Selected recent analyses on flavour physics in high energy processes can be found in [233-240].

\section{Goal 20: Construction of a New Standard Model (NSM)}

Finally, in view of so many parameters present in basically all extensions of the SM like the MSSM, the LHT model and RS models, it is unlikely from my point of view that any of the models studied presently in the literature will turn out in 2026 to be the new model of elementary particle 
physics. On the other hand various structures, concepts and ideas explored these days in the context of specific models may well turn out to be included in the NSM that is predictive, consistent with all the data and giving explanation of observed hierarchies in fermion masses and mixing matrices. While these statements may appear to be very naive, it is a fact that the construction of the NSM is the main goal of elementary particle physics and every theorist, even as old as I am, has a dream that the future NSM will carry her (his) name.

\section{Waiting for Signals of New Physics in FCNC Processes}
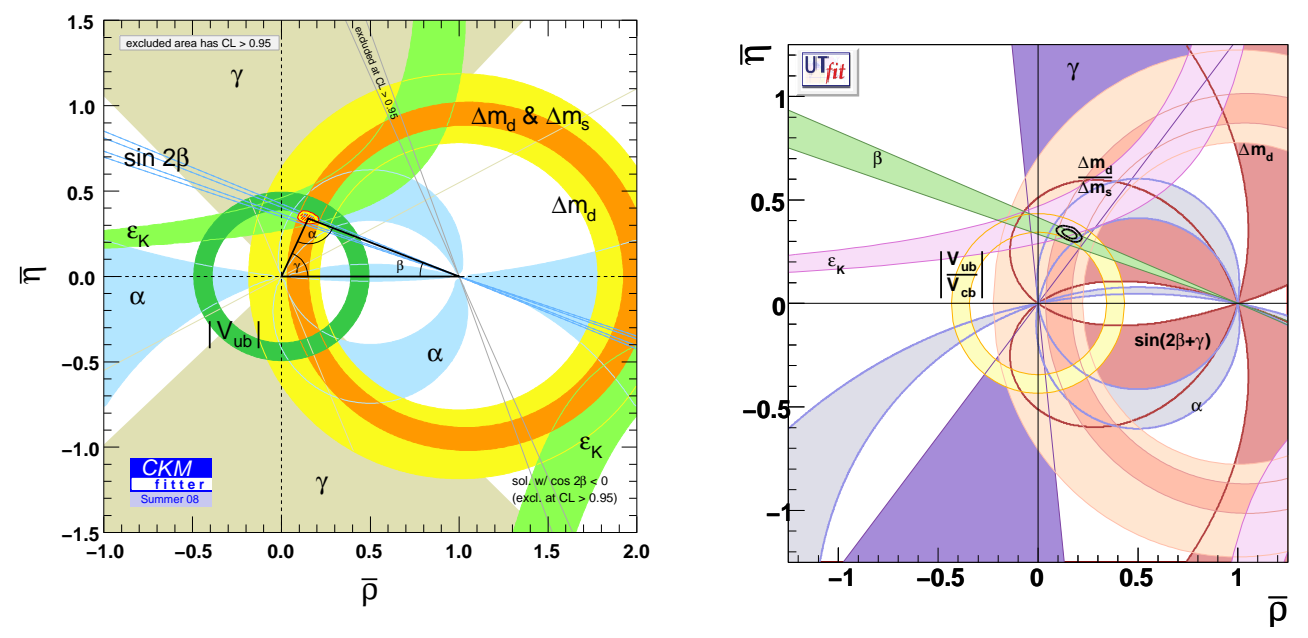

Figure 2: Unitarity triangle fits by the CKMfitter [241] (left) and UTfit [242] (right) collaborations in 2009.

\subsection{A Quick Look at the Status of the CKM Matrix}

The success of the CKM description of flavour violation and in particular CP violation can be best seen by looking at the so-called Unitarity Triangle (UT) fits in Fig.2. The extensive analyses of the UTfit and CKMfitter collaborations [243,244] show that the data on $\left|V_{u s}\right|,\left|V_{u b}\right|,\left|V_{c b}\right|, \varepsilon_{K}, \Delta M_{d}$, $\Delta M_{s}$ and the CP-asymmetry $S_{\psi K_{S}}$, that measures the angle $\beta$ in the UT, are compatible with each other within theoretical and experimental uncertainties. Moreover the angles $\alpha$ and $\gamma$ of the UT determined by means of various non-leptonic decays and sophisticated strategies are compatible with the ones extracted from Fig. 2.

While this agreement is at first sight impressive and many things could already have turned out to be wrong, but they did not, one should remember that only very few theoretically clean observables have been measured precisely so far.

The three parameters relevant for the CKM matrix that have been measured accurately are:

$$
\left|V_{u s}\right|=0.2255 \pm 0.0010, \quad\left|V_{c b}\right|=(41.2 \pm 1.1) \cdot 10^{-3}, \quad \beta=\beta_{\psi K_{S}}=(21.1 \pm 0.9)^{\circ},
$$

where the last number follows from [133]

$$
\sin 2 \beta=0.672 \pm 0.023 \text {. }
$$


It should be mentioned that the value for $\left|V_{c b}\right|$ quoted above results from inclusive and exclusive decays that are not fully consistent with each other. Typically the values resulting from exclusive decays are below $40 \cdot 10^{-3}$. As the value of $\left|V_{c b}\right|$ is very important for FCNC processes in the $K$ system it would be important to clarify this difference which has been with us already for many years. Hopefully, the future Super B facilities in Italy and Japan and new theoretical ideas will provide more precise values. More on this can be found in Bevan's talk [6].

\subsection{Strategies in the Present Decade}

The strategies for the determination of the UT in this decade used basically the following set of fundamental variables:

$$
\left|V_{u s}\right| \equiv \lambda, \quad\left|V_{c b}\right|, \quad R_{t}, \quad \beta
$$

where (see Fig. 3)

$$
R_{t} \equiv \frac{\left|V_{t d} V_{t b}^{*}\right|}{\left|V_{c d} V_{c b}^{*}\right|}=\sqrt{(1-\bar{\rho})^{2}+\bar{\eta}^{2}}=\frac{1}{\lambda}\left|\frac{V_{t d}}{V_{c b}}\right|, \quad V_{t d}=\left|V_{t d}\right| e^{-i \beta} .
$$

Now, $\left|V_{u s}\right|$ and $\left|V_{c b}\right|$ extracted from tree level decays are free from NP pollution. In contrast, $R_{t}$ and $\beta$ in the parameter set in (4.3) can only be extracted from loop-induced FCNC processes and hence are potentially sensitive to NP effects. Consequently, the corresponding UT, the universal unitarity triangle (UUT) [17] of models with CMFV [18,19], could differ from the true UT triangle.

Indeed, within the SM and CMFV models the parameters $R_{t}$ and $\beta$ can be related in the following way to the observables $\Delta M_{s, d}$ and $S_{\psi K_{S}}$

$$
R_{t}=\xi \frac{1}{\lambda} \sqrt{\frac{m_{B_{s}}}{m_{B_{d}}}} \sqrt{\frac{\Delta M_{d}}{\Delta M_{s}}}, \quad \sin 2 \beta=S_{\psi K_{S}},
$$

where $\Delta M_{d}$ and $\Delta M_{s}$ are the mass differences in the neutral $B_{d}$ and $B_{s}$ systems, $S_{\psi K_{s}}$ represents the mixing-induced CP asymmetry in the decay $B_{d} \rightarrow \psi K_{S}$ and the value of the non-perturbative parameter $\xi$ is given as follows

$$
\xi=\frac{\sqrt{\hat{B}_{B_{s}}} F_{B_{s}}}{\sqrt{\hat{B}_{B_{d}}} F_{B_{d}}}=1.21 \pm 0.04, \quad \xi=1.258 \pm 0.033
$$

as summarized by Lubicz and Tarantino [125] and by the HPQCD collaboration [126], respectively.

In the presence of NP however, these relations are modified and one finds

$$
R_{t}=\xi \frac{1}{\lambda} \sqrt{\frac{m_{B_{s}}}{m_{B_{d}}}} \sqrt{\frac{\Delta M_{d}}{\Delta M_{s}}} \sqrt{\frac{C_{B_{s}}}{C_{B_{d}}}}, \quad \sin \left(2 \beta+2 \phi_{B_{d}}\right)=S_{\psi K_{s}},
$$

where the NP phases $\phi_{B_{q}}$ in $B_{q}$ mixing and the parameters $C_{q}(q=d, s)$ are defined through [245]

$$
M_{12}^{q}=\left\langle B_{q}\left|H_{\mathrm{eff}}^{q}\right| \bar{B}_{q}\right\rangle=\left(M_{12}^{q}\right)^{\mathrm{SM}}+\left(M_{12}^{q}\right)^{\mathrm{NP}}=C_{B_{q}} e^{2 i \phi_{B_{q}}}\left(M_{12}^{q}\right)^{\mathrm{SM}} .
$$

For the mass differences in the $B_{q}$ meson system one then has

$$
\Delta M_{q}=2\left|M_{12}^{q}\right|=C_{B_{q}} \Delta M_{q}^{\mathrm{SM}} .
$$


The outcome of using (4.5), $\left|V_{c b}\right|$ and $\left|V_{u s}\right|$ are the parameters $\bar{\rho}$ and $\bar{\eta}$ that presently are given as follows

$$
\begin{aligned}
& \bar{\rho}= \begin{cases}0.154 \pm 0.021 & \text { (UTfit), } \\
0.139_{-0.027}^{+0.025} & \text { (CKMfitter). }\end{cases} \\
& \bar{\eta}= \begin{cases}0.340 \pm 0.013 & \text { (UTfit), } \\
0.341_{-0.015}^{+0.016} & \text { (CKMfitter). }\end{cases}
\end{aligned}
$$

Yet, this determination could be polluted by NP and as we will see below another look at the UT analysis presented below reveals a number of tensions in this determination.

Finally let us stress that the angle $\alpha$ is already well determined from $B_{d} \rightarrow \rho \rho$ and $B_{d} \rightarrow \rho \pi$ decays [133]:

$$
\alpha=(91.4 \pm 4.6)^{\circ} .
$$

A specific analysis employing the mixing induced CP asymmetries $S_{\psi K_{S}}, S_{\rho \rho}$ and the QCDF approach finds [246] $\alpha=(87 \pm 6)^{\circ}$. Summaries of other determinations of $\alpha$ exist [7,9].

\subsection{Unitarity Triangle in the LO Approximation}

Even if NP could have still some visible impact on the determination of the UT presented above, the basic shape of the UT has been determined in this decade and in the LO approximation it can be characterized by two numbers:

$$
\alpha=90^{\circ}, \quad \sin 2 \beta=\frac{2}{3},
$$

implying rather accurately

$$
\begin{gathered}
\beta=21^{\circ}, \quad \gamma=69^{\circ}, \\
\bar{\rho}=\sin \beta \cos \gamma=0.128, \quad \bar{\eta}=\sin \beta \sin \gamma=0.33
\end{gathered}
$$

and

$$
R_{b}=\sin \beta=0.36, \quad R_{t}=\sin (\alpha+\beta)=0.93 .
$$

This is an important achievement of the present decade but in my opinion in the next decade we should proceed in a different manner. First, however let us briefly return to our first goal of the previous section.

\subsection{The Quest for $\left|V_{u b}\right|$ and the Angle $\gamma$}

As we have already stressed in Goal 1 of the previous section, precise measurements of the side $R_{b}\left(\left|V_{u b}\right|\right)$ and of the angle $\gamma$ in the UT of Fig. 3 by means of tree level decays that are independent of any new physics to a good approximation, are undisputably very important.

Indeed the status of $\left|V_{u b}\right|$ and $\gamma$ from tree level decays is not particularly impressive:

$$
\left|V_{u b}\right|= \begin{cases}(4.0 \pm 0.3) \cdot 10^{-3} & \text { (inclusive) } \\ (3.5 \pm 0.4) \cdot 10^{-3} & \text { (exclusive) }\end{cases}
$$




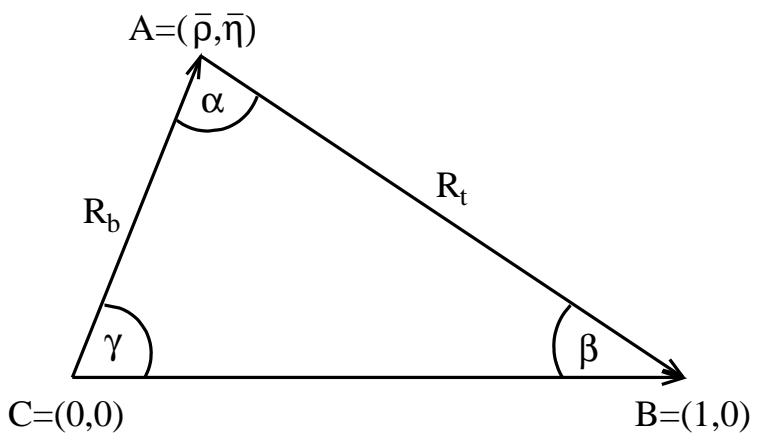

Figure 3: The Unitarity Triangle

$$
\gamma= \begin{cases}(78 \pm 12)^{\circ} & (\text { UTfit }) \\ \left(76_{-23}^{+16}\right)^{\circ} & \text { (CKMfitter) } .\end{cases}
$$

It is very important to precisely measure $\left|V_{u b}\right|$ and $\gamma$ in tree level decays in the future as they determine the so-called reference UT (RUT) [247], that is free from NP pollution. Having determined $\left|V_{u b}\right|$ and $\gamma$ from tree level decays would allow to obtain the CKM matrix without NP pollution, with the four fundamental flavour parameters being now

$$
\left|V_{u s}\right|, \quad\left|V_{u b}\right|, \quad\left|V_{c b}\right|, \quad \gamma,
$$

and to construct the RUT [247] by means of $\lambda=\left|V_{u s}\right|$,

$$
R_{b}=\left(1-\frac{\lambda^{2}}{2}\right) \frac{1}{\lambda}\left|\frac{V_{u b}}{V_{c b}}\right|
$$

and $\gamma$.

This is indeed a very important goal as it would give us immediately the true values of $R_{t}$ and $\beta$ in Fig. 3 by simply using

$$
R_{t}=\sqrt{1+R_{b}^{2}-2 R_{b} \cos \gamma}, \quad \cot \beta=\frac{1-R_{b} \cos \gamma}{R_{b} \sin \gamma} .
$$

Comparing this result with the one obtained by means of (4.5) and using (4.7) would tell us whether $\mathrm{NP}$ in $\Delta B=2$ processes is at work.

\subsection{Strategies for the Search for New Physics in the Next Decade}

Let us first emphasize that until now only $\Delta F=2$ FCNC processes could be used in the UTfits. The measured $B \rightarrow X_{s} \gamma$ and $B \rightarrow X_{s} l^{+} l^{-}$decays and their exclusive counterparts are sensitive to $\left|V_{t s}\right|$ that has nothing to do with the plots in Fig. 2. The same applies to the observables in the $B_{S}$ system, which with the $S_{\psi \phi}$ anomaly observed by CDF and D0 and the studies of rare $B_{s}$ decays at Tevatron and later at LHC are becoming central for flavour physics. Obviously these comments also apply to all lepton flavour violating processes.

In this context a special role is played by $\operatorname{Br}\left(K^{+} \rightarrow \pi^{+} v \bar{v}\right)$ and $\operatorname{Br}\left(K_{L} \rightarrow \pi^{0} v \bar{v}\right)$ as their values allow a theoretically clean construction of the UT in a manner complementary to its present 
determinations [248]: the height of the UT is determined from $\operatorname{Br}\left(K_{L} \rightarrow \pi^{0} v \bar{v}\right)$ and the side $R_{t}$ from $\operatorname{Br}\left(K^{+} \rightarrow \pi^{+} v \bar{v}\right)$. Thus projecting the results of future experimental results for these two branching ratios on the $(\bar{\rho}, \bar{\eta})$ plane could be a very good test of the SM.

Yet, generally I do not think that in the context of the search for the NSM (see Goal 20) it is a good strategy to project the results of all future measurements of rare decays on the $(\bar{\rho}, \bar{\eta})$ plane or any other of five planes related to the remaining unitarity triangles. This would only teach us about possible inconsistences within the SM but would not point towards a particular NP model.

In view of this, here comes a proposal for the strategy for searching for NP in the next decade, in which hopefully $R_{b}$ and $\gamma$ will be precisely measured, CP violation in the $B_{s}$ system explored and many goals listed in the previous section reached.

This strategy, which is a summary of many ideas present already in the literature, proceeds in three steps:

\section{Step 1}

In order to study transparently possible tensions between $\varepsilon_{K}, \sin 2 \beta,\left|V_{u b}\right|, \gamma$ and $R_{t}$ let us leave the $(\bar{\rho}, \bar{\eta})$ plane and go to the $R_{b}-\gamma$ plane [249] suggested already several years ago and recently strongly supported by the analyses in $[55,250]$. The $R_{b}-\gamma$ plane is shown in Fig. 4 . We will explain this figure in the next subsection.

\section{Step 2}

In order to search for NP in rare $K, B_{d}, B_{s}, D$ decays, in CP violation in $B_{s}$ and charm decays, in LFV decays, in EDM's and $(g-2)_{\mu}$ let us go to specific plots that exhibit correlations between various observables. As we will see below such correlations will be crucial to distinguish various NP scenarios. Of particular importance are the correlations between the CP asymmetry $S_{\psi \phi}$ and $B_{s} \rightarrow \mu^{+} \mu^{-}$, between the anomalies in $S_{\phi K_{s}}$ and $S_{\psi \phi}$, between $K^{+} \rightarrow \pi^{+} v \bar{v}$ and $K_{L} \rightarrow \pi^{0} v \bar{v}$, between $K^{+} \rightarrow \pi^{+} \nu \bar{v}$ and $S_{\psi \phi}$, between $S_{\phi K_{s}}$ and $d_{e}$, between $S_{\psi \phi}$ and $(g-2)_{\mu}$ and also those involving lepton flavour violating decays.

\section{Step 3}

In order to monitor the progress made in the next decade when additional data on flavour changing processes will become available, it is useful to construct a "DNA-Flavour Test" of NP models [55] including Supersymmetry, the LHT model, the RSc and various supersymmetric flavour models and other models, with the aim to distinguish between these NP scenarios in a global manner.

Having this strategy in mind we will in the rest of this writing illustrate it on several examples.

\subsection{The $\varepsilon_{\mathrm{K}}$-Anomaly and Related Tensions}

The $\mathrm{CP}$-violating parameter $\varepsilon_{K}$ in the $\mathrm{SM}$ is given as follows

$$
\left|\varepsilon_{K}\right|^{\mathrm{SM}}=\kappa_{\varepsilon} C_{\varepsilon} \hat{B}_{K}\left|V_{c b}\right|^{2}\left|V_{u s}\right|^{2}\left(\frac{1}{2}\left|V_{c b}\right|^{2} R_{t}^{2} \sin 2 \beta \eta_{t t} S_{0}\left(x_{t}\right)+R_{t} \sin \beta\left(\eta_{c t} S_{0}\left(x_{c}, x_{t}\right)-\eta_{c c} x_{c}\right)\right),
$$

where $C_{\varepsilon}$ is a numerical constant and the SM loop functions $S_{0}$ depend on $x_{i}=m_{i}^{2}\left(m_{i}\right) / M_{W}^{2}$. The factors $\eta_{i j}$ are QCD corrections known at the NLO level [251-254], $\hat{B}_{K}$ is a non-perturbative parameter and $\kappa_{\varepsilon}$ is explained below.

Until the discovery of CP violation in the $B_{d}$ system, $\varepsilon_{K}$ played the crucial role in tests of CP violation, but after the precise measurements of $\sin 2 \beta$ and of the ratio $\Delta M_{d} / \Delta M_{s}$ its role in the 

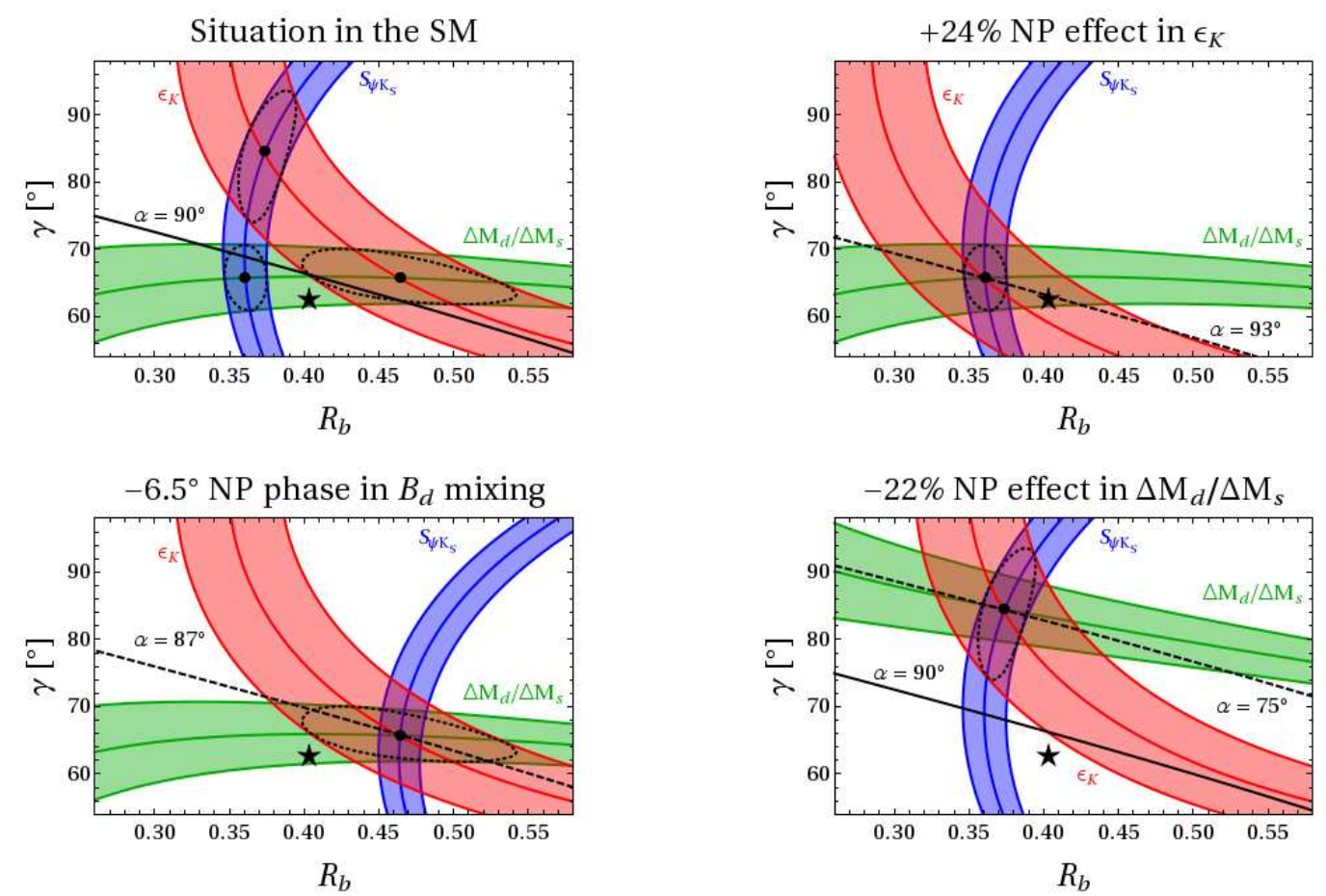

Figure 4: The $R_{b}-\gamma$ plane as discussed in the text. For further explanations see [55]

CKM fits declined because of the large error in $\hat{B}_{K}$. Also for this reason the size of CP violation in the $K$ and $B$ systems were commonly declared to be compatible with each other within the SM.

This situation changed in 2008 due to the improved value of $\hat{B}_{K}$, the improved determinations of the elements of the CKM matrix and in particular due to the inclusion of additional corrections to $\varepsilon_{K}$ [255] that were neglected in the past, enhancing the role of this CP-violating parameter in the search for NP.

Indeed it has been recently stressed [255] that the SM prediction for $\varepsilon_{K}$ implied by the measured value of $\sin 2 \beta$ may be too small to agree with experiment. The main reasons for this are on the one hand a decreased value of $\hat{B}_{K}=0.724 \pm 0.008 \pm 0.028$ [256] (see also [257]), lower by 5-10\% with respect to the values used in UT fits until recently and on the other hand the decreased value of $\varepsilon_{K}$ in the SM arising from a multiplicative factor, estimated as $\kappa_{\varepsilon}=0.92 \pm 0.02[255,258]$. Earlier discussions of such corrections can be found in [259-261].

Given that $\varepsilon_{K} \propto \hat{B}_{K} \kappa_{\varepsilon}$, the total suppression of $\varepsilon_{K}$ compared to the commonly used formulae is typically of order $15-20 \%$ and using (4.1), (4.2) and (4.5) one finds now [258]

$$
\left|\varepsilon_{K}\right|^{\mathrm{SM}}=(1.78 \pm 0.25) \times 10^{-3},
$$

to be compared to the experimental measurement [262]

$$
\left|\varepsilon_{K}\right|^{\exp }=(2.229 \pm 0.010) \times 10^{-3} .
$$

The $15 \%$ error in (4.19) arises from the three main sources of uncertainty that are still $\hat{B}_{K},\left|V_{c b}\right|^{4}$ and $R_{t}^{2}$. However, it should be stressed that $\hat{B}_{K}$ known by now with $4 \%$ accuracy is not the main uncertainty which now is dominantly due to $\left|V_{c b}\right|$ and in the ballpark of $10 \%$. 
As seen in (4.18) the agreement between the SM and (4.20) improves for higher values of $\hat{B}_{K}, R_{t}$ or $\left|V_{c b}\right|$ and also the correlation between $\varepsilon_{K}$ and $\sin 2 \beta$ within the SM is highly sensitive to these parameters. Consequently improved determinations of all these parameters are very desirable in order to find out whether NP is at work in $S_{\psi K_{S}}$ or in $\varepsilon_{K}$ or both. Some ideas can be found in $[255,258,263,264]$.

The tension in question can be also seen in the most recent fit of the UTfit collaboration shown in Fig. 2, which now also includes the $\kappa_{\varepsilon}$ correction. In order to see this more transparently let us have now a look at the $R_{b}-\gamma$ plane in Fig. 4 taken from [55], where details on input parameters can be found. There, in the upper left plot the blue (green) region corresponds to the $1 \sigma$ allowed range for $\sin 2 \beta\left(R_{t}\right)$ as calculated by means of (4.5). The red region corresponds to $\left|\varepsilon_{K}\right|$ as obtained by equating (4.18) with (4.20). Finally the solid black line corresponds to $\alpha=90^{\circ}$ that is close to the value favoured by UT fits and the determination from $B \rightarrow \rho \rho$ [246].

It is evident that there is a tension between various regions as there are three different values of $\left(R_{b}, \gamma\right)$, dependending on which two constraints are simultaneously applied. The four immediate solutions to this tension are as follows:

1. There is a positive NP effect in $\varepsilon_{K}$ while $\sin 2 \beta$ and $\Delta M_{d} / \Delta M_{s}$ are SM-like [255], as shown by the upper right plot of Fig. 4 . The required effect in $\varepsilon_{K}$ could be for instance achieved within models with CMFV by a positive shift in the function $S_{0}\left(x_{t}\right)$ [258] which, while not modifying $(\sin 2 \beta)_{\psi K_{S}}$ and $\Delta M_{d} / \Delta M_{s}$, would require the preferred values of $\sqrt{\hat{B}_{B_{d, s}}} F_{B_{d, s}}$ to be by $\simeq 10 \%$ lower than the present central values in order to fit $\Delta M_{d}$ and $\Delta M_{s}$ separately. Alternatively, new non-minimal sources of flavour violation relevant only for the $K$ system could solve the problem. Note that this solution corresponds to $\gamma \simeq 66^{\circ}, R_{b} \simeq 0.36$ and $\alpha \simeq 93^{\circ}$ in accordance with the usual UT analysis.

2. $\varepsilon_{K}$ and $\Delta M_{d} / \Delta M_{s}$ are NP free while $S_{\psi K_{S}}$ is affected by a NP phase $\phi_{B_{d}}$ in $B_{d}$ mixing of approximately $-7^{\circ}$ as indicated in (4.7) and shown by the lower left plot of Fig. 4. The predicted value for $\sin 2 \beta$ is now shifted to $\sin 2 \beta \approx 0.85$ [255, 258,263,264]. This value is significantly larger than the measured $S_{\psi K_{S}}$ which allows to fit the experimental value of $\varepsilon_{K}$. Note that this solution is characterized by a large value of $R_{b} \simeq 0.47$, that is significantly larger than its exclusive determinations but still compatible with the inclusive determinations. The angles $\gamma \simeq 66^{\circ}$ and $\alpha \simeq 87^{\circ}$ agree with the usual UT analysis.

3. $\varepsilon_{K}$ and $S_{\psi K_{S}}$ are NP free while the determination of $R_{t}$ through $\Delta M_{d} / \Delta M_{s}$ is affected by $\mathrm{NP}$ as indicated in (4.7) and shown by the lower right plot of Fig. 4. In that scenario one finds $\Delta M_{d}^{\mathrm{SM}} / \Delta M_{s}^{\mathrm{SM}}$ to be much higher than the actual measurement. In order to agree exactly with the experimental central value, one needs a NP contribution to $\Delta M_{d} / \Delta M_{s}$ at the level of $-22 \%$. Non-universal contributions suppressing $\Delta M_{d}\left(C_{B_{d}}<1\right)$ and/or enhancing $\Delta M_{s}\left(C_{B_{s}}>1\right)$ could be responsible for this shift as is evident from (4.7). The increased value of $R_{t}$ that compensates the negative effect of NP in $\Delta M_{d} / \Delta M_{s}$ allows to fit the experimental value of $\varepsilon_{K}$. This solution is characterized by a large value of $\gamma \simeq 84^{\circ}$ and $\alpha$ much below $90^{\circ}$. The latter fact could become problematic for this solution when the determination of $\alpha$ further improves.

4. The value of $\left|V_{c b}\right|$ is significantly increased to roughly $43.5 \cdot 10^{-3}$, which seems rather unlikely.

The first three NP scenarios characterized by black points in Fig. 4 will be clearly distinguished 
from each other once the values of $\gamma$ and $R_{b}$ from tree level decays will be precisely known. Moreover, if future measurements of $\left(R_{b}, \gamma\right)$ will select a point in the $R_{b}-\gamma$ plane that differs from the black points in Fig. 4 , it is likely that NP will simultaneously enter $\varepsilon_{K}, S_{\psi K_{S}}$ and $\Delta M_{d} / \Delta M_{s}$. It will be interesting to monitor future progress in the $R_{b}-\gamma$ plane.

Finally, let us mention that the tensions discussed above could be in principle somewhat reduced through penguin contributions to $B \rightarrow \psi K_{S}[265,266]$. However a different view has been expressed in [267], where such effects have been found to be negligible.

\subsection{Rare Decays $\mathbf{K}^{+} \rightarrow \pi^{+} v \bar{v}$ and $\mathbf{K}_{\mathbf{L}} \rightarrow \pi^{\mathbf{0}} v \bar{v}$}

Let us next discuss in more detail two most popular decays among rare $K$ decays: $K^{+} \rightarrow \pi^{+} v \bar{v}$ and $K_{L} \rightarrow \pi^{0} v \bar{v}$. These decays are theoretically very clean and very sensitive to NP contributions in $Z$ penguin diagrams. It is then not surprising that theorists invested over 25 years to improve the SM prediction and to analyze these decays in many extensions of the SM. The most recent predictions that include NNLO QCD corrections and electroweak corrections read [35, 36]

$$
\begin{gathered}
\operatorname{Br}\left(K^{+} \rightarrow \pi^{+} v \bar{v}\right)_{\mathrm{SM}}=(8.5 \pm 0.7) \cdot 10^{-11}, \\
\operatorname{Br}\left(K_{L} \rightarrow \pi^{0} v \bar{v}\right)_{\mathrm{SM}}=(2.8 \pm 0.6) \cdot 10^{-11},
\end{gathered}
$$

where the errors are dominated by parametrical uncertainties, in particular by the CKM parameters. In the past a sizable uncertainty in $\operatorname{Br}\left(K^{+} \rightarrow \pi^{+} v \bar{v}\right)$ was due to the charm quark mass. But presently $m_{c}$ is known to be $m_{c}\left(m_{c}\right)=1.279 \pm 0.013 \mathrm{GeV}$ [127] and this uncertainty is basically eliminated. Also very significant progress has been made in estimating non-perturbative contributions to the charm component [167] and in the determination of the relevant hadronic matrix elements from tree level leading $K$ decays [168]. Reviews of these two decays can be found in [268-272].

On the experimental side seven events of $K^{+} \rightarrow \pi^{+} v \bar{v}$ decay have been observed by E787 and E949 at Brookhaven resulting in [273]

$$
\operatorname{Br}\left(K^{+} \rightarrow \pi^{+} v \bar{v}\right)=\left(17.3_{-10.5}^{+11.5}\right) \cdot 10^{-11}
$$

The experimental upper bound on $\operatorname{Br}\left(K_{L} \rightarrow \pi^{0} v \bar{v}\right)$ is still by more than two orders of magnitude above the SM value in (4.22) but the present upper bound from E391a at KEK [274] of $B r\left(K_{L} \rightarrow\right.$ $\left.\pi^{0} v \bar{v}\right) \leq 6.7 \cdot 10^{-8}$ should be significantly improved in the coming decade. Experimental prospects for both decays have been already mentioned in connection with Goal 10 on our list.

Once measured, these decays will provide a very clean determination of the angle $\beta$ in the UT as some parametric uncertainties, in particular the value of $\left|V_{c b}\right|$, cancel out in this determination. This implies one of the golden relation of MFV [248,275] that connects $K$ and $B$ physics,

$$
(\sin 2 \beta)_{S_{\psi K_{S}}}=(\sin 2 \beta)_{K_{L} \rightarrow \pi^{0} v \bar{v}}
$$

which can be strongly violated in models with new flavour and CP-violating interactions, such as the LHT model [73, 276] and the RSc model analyzed in [83]. 


\begin{tabular}{|c|c|c|c|c|}
\hline Model/Observable & $\operatorname{Br}\left(K^{+} \rightarrow \pi^{+} v \bar{v}\right)$ & $\operatorname{Br}\left(K_{L} \rightarrow \pi^{0} v \bar{v}\right)$ & $\operatorname{Br}\left(B_{s} \rightarrow \mu^{+} \mu^{-}\right)$ & $S_{\psi \phi}$ \\
\hline CMFV & $20 \%$ & $20 \%$ & $20 \%$ & 0.04 \\
MFV & $30 \%$ & $30 \%$ & $1000 \%$ & 0.04 \\
AC & $2 \%$ & $2 \%$ & $1000 \%$ & 1.0 \\
RVV2 & $10 \%$ & $10 \%$ & $1000 \%$ & 0.50 \\
AKM & $10 \%$ & $10 \%$ & $1000 \%$ & 0.30 \\
$\delta$ LL & $2 \%$ & $2 \%$ & $1000 \%$ & 0.04 \\
FBMSSM & $2 \%$ & $2 \%$ & $1000 \%$ & 0.04 \\
GMSSM & $300 \%$ & $500 \%$ & $1000 \%$ & 1.0 \\
LHT & $150 \%$ & $200 \%$ & $30 \%$ & 0.30 \\
RSc & $60 \%$ & $150 \%$ & $10 \%$ & 0.75 \\
\hline
\end{tabular}

Table 1: Approximate maximal enhancements for various observables in different models of NP. In the case of $S_{\psi \phi}$ we give the maximal positive values. The NP models have been defined in Section 2.4.

While the test of the relation (4.24) in future experiments will tell us whether some NP disturbs this MFV correlation, in order to identify which NP is at work we have to do much more and consider other decays and observables.

To this end let us first list the maximal enhancements of these two branching ratios in a number of NP scenarios. These are given in the second and third column of Table 1, where $100 \%$ means an enhancement of the branching ratio by a factor of two. These enhancements in a given NP scenario are consistent with all existing data but could be significantly decreased through various correlations when new observables will be measured.

A striking hierarchy of enhancements is exhibited in this table:

- In the GMSSM still very large enhancements are possible. More modest but still large enhancements are possible in the LHT model [73, 276] and in the RSc model [83]. In the GMSSM and the LHT model the central experimental value of $\operatorname{Br}\left(K^{+} \rightarrow \pi^{+} v \bar{v}\right)$ in (4.23) can be reproduced. In the RSc model values above $15 \times 10^{-11}$ are rather unlikely.

- Enhancements of both branching ratios in CMFV and MFV scenarios are small, but as the theory is very clean, powerful experiments will be able to distinguish these NP scenarios on the basis of these decays one day. Yet, the confirmation of the central value for $\operatorname{Br}\left(K^{+} \rightarrow\right.$ $\left.\pi^{+} v \bar{v}\right)$ in (4.23) with a precision of $10 \%$ would certainly tell us that non-MFV interactions are at work.

- The branching ratios for both decays in supersymmetric flavour models considered in subsequent subsections are basically indistinguishable from the SM predictions for $K \rightarrow \pi v \bar{v}$ decays, but as we will see soon these models perform quite differently in the $B_{s}$ system or more explicitly in $b \rightarrow s$ transitions, both $\mathrm{CP}$-conserving and $\mathrm{CP}$-violating.

\subsection{The VIP's of $\mathbf{B}_{\mathbf{s}}$ Physics: $\mathbf{B}_{\mathrm{s}, \mathrm{d}} \rightarrow \mu^{+} \mu^{-}$and $\mathbf{S}_{\psi \phi}$}

We will move now to discuss Goals 6 and 4 in more detail. These goals are in my opinion the most important goals in quark flavour physics until the next EPS11 conference, to be joined 
later by $K \rightarrow \pi v \bar{v}$ decays so that EPS13 will indeed have them all. We will first discuss these two goals separately. Subsequently we will have a grand simultaneous look at $S_{\psi \phi}, B_{s} \rightarrow \mu^{+} \mu^{-}$, $K^{+} \rightarrow \pi^{+} v \bar{v}$ and $K_{L} \rightarrow \pi^{0} v \bar{v}$ that we have already anticipated when constructing Table 1 .

\subsection{1 $\operatorname{Br}\left(\mathbf{B}_{\mathbf{s}, \mathbf{d}} \rightarrow \mu^{+} \mu^{-}\right)$}

One of the main targets of flavour physics in the coming years will be the measurement of the branching ratio for the highly suppressed decay $B_{s} \rightarrow \mu^{+} \mu^{-}$. Hopefully the even more suppressed decay $B_{d} \rightarrow \mu^{+} \mu^{-}$will be discovered as well. These two decays are helicity suppressed in the SM and CMFV models. Their branching ratios are proportional to the squares of the corresponding weak decay constants that suffer still from sizable uncertainties as discussed in the context of Goal 2. However using simultaneously the SM expressions for the very well measured mass differences $\Delta M_{s, d}$, this uncertainty can be eliminated [277] leaving the uncertainties in the hadronic parameters $\hat{B}_{B_{s}}$ and $\hat{B}_{B_{d}}$ as the only theoretical uncertainty in $\operatorname{Br}\left(B_{s, d} \rightarrow \mu^{+} \mu^{-}\right)$. As seen in (3.3) these parameters are already known from lattice calculations [125] with precision of $10 \%$ and enter the branching ratios linearly.

Explicitly we have in the SM [277]

$$
\operatorname{Br}\left(B_{q} \rightarrow \mu^{+} \mu^{-}\right)=C \frac{\tau\left(B_{q}\right)}{\hat{B}_{B_{q}}} \frac{Y^{2}\left(x_{t}\right)}{S\left(x_{t}\right)} \Delta M_{q}, \quad(q=s, d)
$$

with

$$
C=6 \pi \frac{\eta_{Y}^{2}}{\eta_{B}}\left(\frac{\alpha}{4 \pi \sin ^{2} \theta_{W}}\right)^{2} \frac{m_{\mu}^{2}}{M_{\mathrm{W}}^{2}}=4.39 \cdot 10^{-10} .
$$

$S\left(x_{t}\right)=2.32 \pm 0.07$ and $Y\left(x_{t}\right)=0.94 \pm 0.03$ are the relevant top mass dependent one-loop functions. More generally we have in CMFV models

$$
\frac{B r\left(B_{q} \rightarrow \mu \bar{\mu}\right)}{\Delta M_{q}}=4.4 \cdot 10^{-10} \frac{\tau\left(B_{q}\right)}{\hat{B}_{q}} F(v), \quad F(v)=\frac{Y^{2}(v)}{S(v)},
$$

with $Y(v)$ and $S(v)$ replacing $Y\left(x_{t}\right)$ and $S\left(x_{t}\right)$ in a given CMFV model. Using these expressions one finds in the SM the rather precise predictions

$$
\operatorname{Br}\left(B_{s} \rightarrow \mu^{+} \mu^{-}\right)=(3.6 \pm 0.4) \cdot 10^{-9}, \quad B r\left(B_{d} \rightarrow \mu^{+} \mu^{-}\right)=(1.1 \pm 0.1) \cdot 10^{-10} .
$$

These predictions should be compared to the 95\% C.L. upper limits from CDF [278] and D0 [279] (in parentheses)

$$
\operatorname{Br}\left(B_{s} \rightarrow \mu^{+} \mu^{-}\right) \leq 3.3(5.3) \cdot 10^{-8}, \quad B r\left(B_{d} \rightarrow \mu^{+} \mu^{-}\right) \leq 1 \cdot 10^{-8} .
$$

The numbers given above are updates presented at this conference. More information is given by Giovanni Punzi. It is clear from (4.28) and (4.29) that a lot of room is still left for NP contributions.

Now, irrespectively of large uncertainties in the separate SM predictions for $B_{s, d} \rightarrow \mu^{+} \mu^{-}$and $\Delta M_{s, d}$, there exists a rather precise relation between these observables that can be considered as one of the theoretically cleanest predictions of CMFV. This golden relation reads [277]

$$
\frac{B r\left(B_{s} \rightarrow \mu^{+} \mu^{-}\right)}{B r\left(B_{d} \rightarrow \mu^{+} \mu^{-}\right)}=\frac{\hat{B}_{B_{d}}}{\hat{B}_{B_{s}}} \frac{\tau\left(B_{s}\right)}{\tau\left(B_{d}\right)} \frac{\Delta M_{s}}{\Delta M_{d}} r
$$



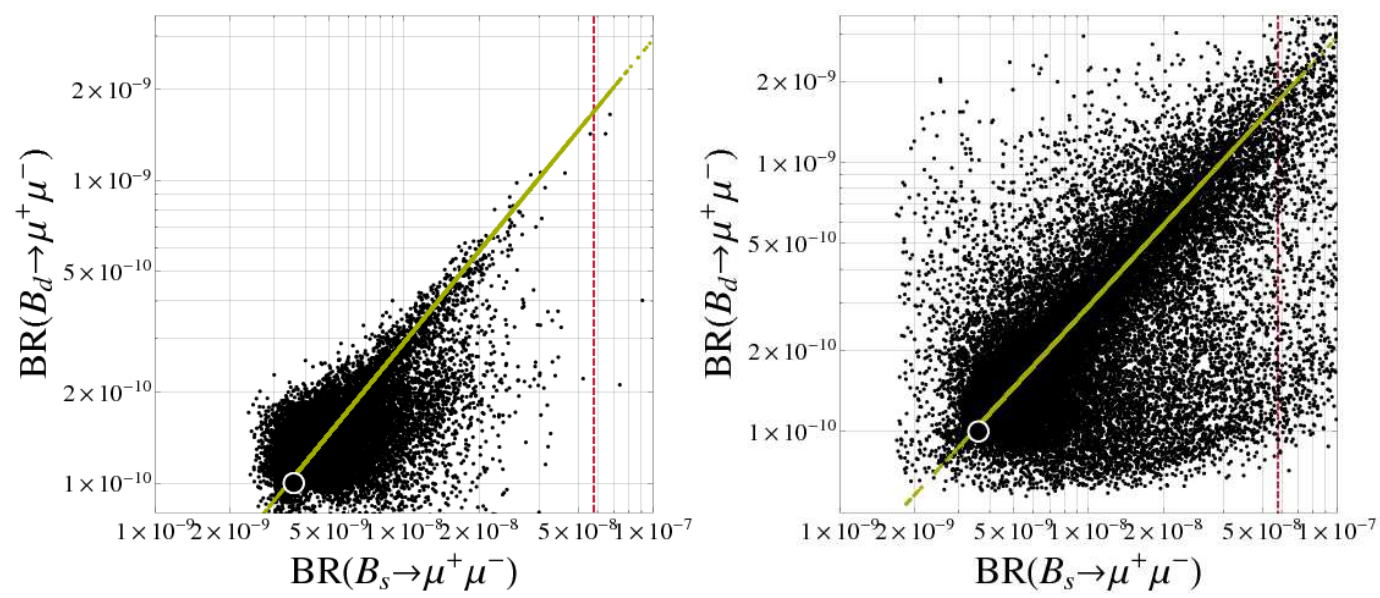

Figure 5: $B_{d, s} \rightarrow \mu^{+} \mu^{-}$branching ratios in the RVV2 model (left) and the $\delta$ LL model (right) as obtained in [55].

with $r=1$ in CMFV models but generally different from unity. For instance in the LHT model one finds $0.3 \leq r \leq 1.6[73,276]$, while in the RSc model $0.6 \leq r \leq 1.3$ [83]. Also in supersymmetric models discussed below $r$ can deviate strongly from unity.

It should be stressed that the ratio $\hat{B}_{B_{d}} / \hat{B}_{B_{s}}=1.00 \pm 0.03$ [125] constitutes the only theoretical uncertainty in (4.30). The remaining quantities entering (4.30) can be obtained directly from experimental data. The right hand side is already known rather precisely: $32.5 \pm 1.7$, but it will still take some time before the left hand side will be known with comparable precision unless NP enhances both branching ratios by an order of magnitude. In the latter case one will very likely find $r \neq 1$ as within CMFV models such large enhancements of $\operatorname{Br}\left(B_{s, d} \rightarrow \mu^{+} \mu^{-}\right)$are not possible.

Large contributions to the branching ratios in question can come from neutral scalar exchanges (Higgs penguins) $[280,281]$ in which case new scalar operators are generated and the helicity suppression is lifted. Thus large enhancements of $B_{s, d} \rightarrow \mu^{+} \mu^{-}$are only possible in the models placed in the entries $(1,2)$ and $(2,2)$ of the flavour matrix in Fig. 1. The prime example here is the MSSM at large $\tan \beta$, in which still in $2002 \operatorname{Br}\left(B_{s} \rightarrow \mu^{+} \mu^{-}\right)$could be as large as $10^{-6}$. The impressive progress by CDF and D0 collaborations, leading to a decrease of the corresponding upper bound by two orders of magnitude totally excluded this possibility but there is still hope that a clear signal of NP at the level of $\mathscr{O}\left(10^{-8}\right)$ will be seen in these decays. We will discuss a number of SUSY predictions below, where such enhancements are still possible.

In the MSSM with MFV and large $\tan \beta$ there is a strong correlation between $\operatorname{Br}\left(B_{s, d} \rightarrow\right.$ $\mu^{+} \mu^{-}$) and $\Delta M_{s}$ [282-286] implying that an enhancement of these branching ratios with respect to the SM is correlated with a suppression of $\Delta M_{s}$ below the SM value. In fact the MSSM with MFV was basically the only model that "predicted" the suppression of $\Delta M_{s}$ below the SM prediction as seemed to be the case just after the discovery of the $B_{s}^{0}-\bar{B}_{s}^{0}$ mixing. Meanwhile the lattice values for weak decay constants changed and there is no suppression relativ to $\left(\Delta M_{s}\right)_{\mathrm{SM}}$ seen within theoretical uncertainties in the data. With the decrease of the experimental upper bound on $\operatorname{Br}\left(B_{s, d} \rightarrow \mu^{+} \mu^{-}\right)$also in the MSSM with MFV the predicted suppression of $\Delta M_{s}$ amounts to at most $10 \%$ and it will require a considerable reduction of the lattice uncertainties in the evaluation 
of $\left(\Delta M_{s}\right)_{\mathrm{SM}}$ before the correlation in question can be verified or falsified by experiment. As we will see soon, in the MSSM with non-MFV interactions the correlation discussed here is absent [55]. Other analyses of this issue can be found in $[65,287,288]$ and a review on Higgs penguins can be found in [289]. Also in models with hybrid gauge-gravity mediation the MFV-like correlattion in question can be strongly modified [290].

Looking at $\operatorname{Br}\left(B_{s, d} \rightarrow \mu^{+} \mu^{-}\right)$in CMFV, MFV, LHT, RSc, GMSSM and the specific supersymmetric flavour models AC, RVV2, AKM, $\delta$ LL and FBMSSM we identify a striking hierarchy of possible enhancements that is, as seen in table 1, opposite to the one found in the case of $K \rightarrow \pi v \bar{v}$ decays. An exception to this pattern is GMSSM:

- In the GMSSM, SUSY with MFV and all SUSY flavour models $\operatorname{Br}\left(B_{s, d} \rightarrow \mu^{+} \mu^{-}\right)$can still reach the present experimental bounds because of the presence of Higgs penguins that become very important at large $\tan \beta:$ a $(\tan \beta)^{6}$ enhancement of the branching ratios is present in this case.

- In CMFV, the LHT and the RSc only enhancements of 20\%, 30\% and $10 \%$ are possible $[73,83,276]$ as Higgs penguins are irrelevant here and the Z-penguins in spite of non-MFV interactions in the case of the LHT and the RSc do not lift the helicity suppression. Moreover the custodial protection of left-handed Z couplings in the RSc allows only right-handed Z couplings to be relevant and these cannot do much in this case [83].

Recently a closer look at $\operatorname{Br}\left(B_{s, d} \rightarrow \mu^{+} \mu^{-}\right)$has been made in the context of specific SUSY flavour models such as AC, RVV2, AKM, $\delta$ LL showing that the measurement of both branching ratios $\operatorname{Br}\left(B_{s, d} \rightarrow \mu^{+} \mu^{-}\right)$can not only test the golden MFV relation in (4.30) but also give some insight in different SUSY flavour models. We find [55]:

- The ratio $\operatorname{Br}\left(B_{d} \rightarrow \mu^{+} \mu^{-}\right) / \operatorname{Br}\left(B_{s} \rightarrow \mu^{+} \mu^{-}\right)$in the AC and RVV2 models is dominantly below its CMFV prediction in (4.30) and can be much smaller than the latter. In the AKM model this ratio stays much closer to the MFV value of roughly $1 / 33$ [53,277] and can be smaller or larger than this value with equal probability. Still, values of $\operatorname{Br}\left(B_{d} \rightarrow \mu^{+} \mu^{-}\right)$ as high as $1 \times 10^{-9}$ are possible in all these models as $\operatorname{Br}\left(B_{s} \rightarrow \mu^{+} \mu^{-}\right)$can be strongly enhanced. We show this in the case of the RVV2 model in the left plot of Fig. 5.

- Interestingly, in the $\delta$ LL-models, the ratio $\operatorname{Br}\left(B_{d} \rightarrow \mu^{+} \mu^{-}\right) / \operatorname{Br}\left(B_{s} \rightarrow \mu^{+} \mu^{-}\right)$can not only deviate significantly from its CMFV value, but in contrast to the models with right-handed currents considered by us can also be much larger that the MFV value. Consequently, $\operatorname{Br}\left(B_{d} \rightarrow \mu^{+} \mu^{-}\right)$as high as $(1-2) \times 10^{-9}$ is still possible while being consistent with the bounds on all other observables, in particular the one on $\operatorname{Br}\left(B_{s} \rightarrow \mu^{+} \mu^{-}\right)$. We show this in the right plot of Fig. 5 .

\subsubsection{The $S_{\psi \phi}$ Asymmetry}

The tiny complex phase of the element $V_{t s}$ in the CKM matrix precludes any sizable CP violating effects in the decays of the $B_{s}$ mesons within the SM and models with MFV. In particular the very clean mixing induced asymmetry $S_{\psi \phi}$ is predicted to be

$$
\left(S_{\psi \phi}\right)_{\mathrm{SM}}=\sin \left(2\left|\beta_{S}\right|\right) \approx 0.04
$$




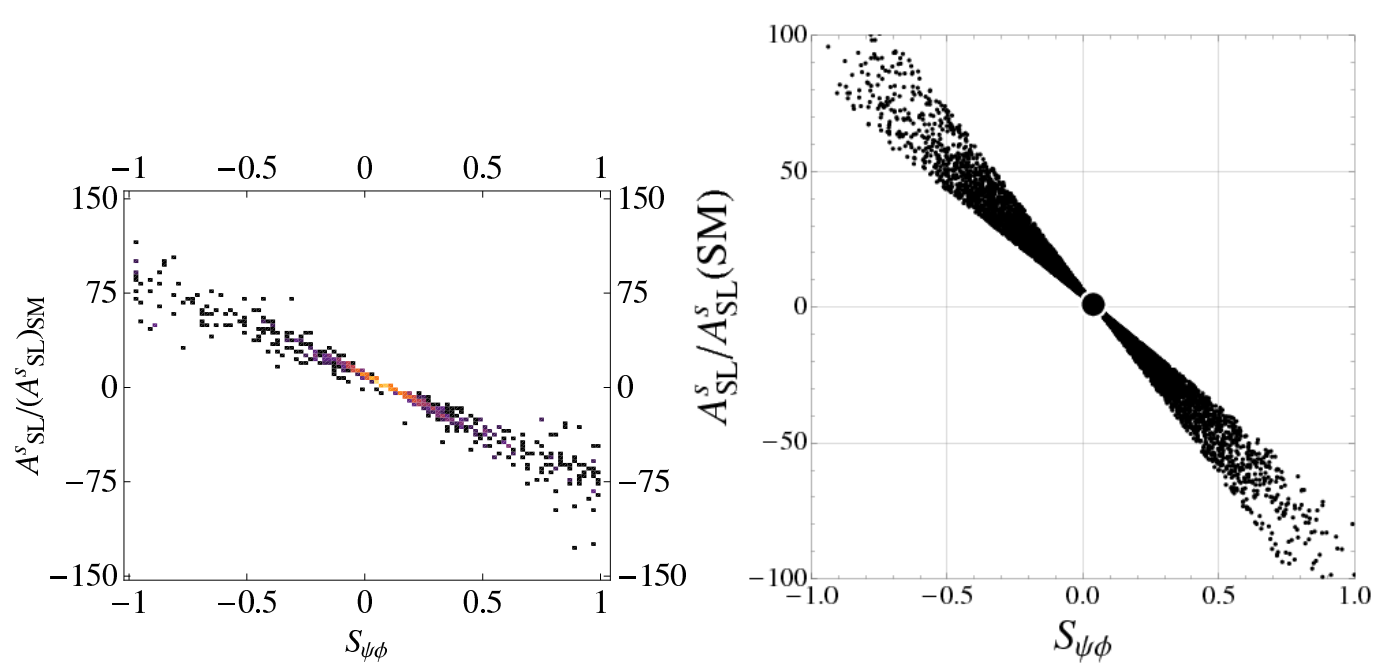

Figure 6: $A_{S L}^{S}$ vs. $S_{\psi \phi}$ in the RSc model (left) [82] and in the AC model (right) [55].

with $-\beta_{s}$ being the phase of $V_{t s}$. As pointed out in [291] some hadronic uncertainties not discussed in the past could still be non-negligible so that values of $S_{\psi \phi}$ as high as 0.1 could not be immidiately considered as signals of NP. However the same paper proposes various strategies to overcome these difficulties through additional measurements of different decay channels that will be available in the coming years.

In the presence of new physics (4.31) is modified as follows [19],

$$
S_{\psi \phi}=\sin \left(2\left|\beta_{s}\right|-2 \phi_{B_{s}}\right),
$$

where $\phi_{B_{s}}$ is a new phase in $B_{s}^{0}-\bar{B}_{s}^{0}$ mixing as defined in (4.8).

Already in 2006 Lenz and Nierste [292], analyzing D0 and CDF data pointed out some hints for a large phase $\phi_{B_{s}}$. In 2008 new hints appeared, emphasized in particular by the UTfit collaboration [293]. The most recent messages from CDF and D0 [294] imply a $2.7 \sigma$ deviation from the SM prediction and we have to wait for higher statistics in order to conclude that NP is at work here [295]. Explicitly CDF and D0 find [133]

$$
S_{\psi \phi} \approx 0.81_{-0.32}^{+0.12}
$$

As the central value of the measured $S_{\psi \phi}$ is around 0.8 , that is one order of magnitude larger than the SM value, the confirmation of this high value in the future would be a spectacular confirmation of non-MFV interactions at work. As demonstrated recently such large values can easily be found in the RSc model [82] and the same comment applies to the GMSSM. The most likely values for $S_{\psi \phi}$ in the LHT do not exceed 0.3 [73] and finding this asymmetry as high as 0.4 would be in favour of the RSc and the GMSSM. Similarly the supersymmetric flavour models with significant right-handed currents (AC, RVV2, AKM) provide sizable enhancements. Here the double Higgs penguin contributing to $M_{12}^{s}$ is at work. The following hierarchy in maximal values is found (see Table 1)

$$
\left(S_{\psi \phi}\right)_{\mathrm{LHT}}^{\max } \approx\left(S_{\psi \phi}\right)_{\mathrm{AKM}}^{\max }<\left(S_{\psi \phi}\right)_{\mathrm{RVV} 2}^{\max }<\left(S_{\psi \phi}\right)_{\mathrm{RSc}}^{\max } \approx\left(S_{\psi \phi}\right)_{\mathrm{AC}}^{\max }
$$




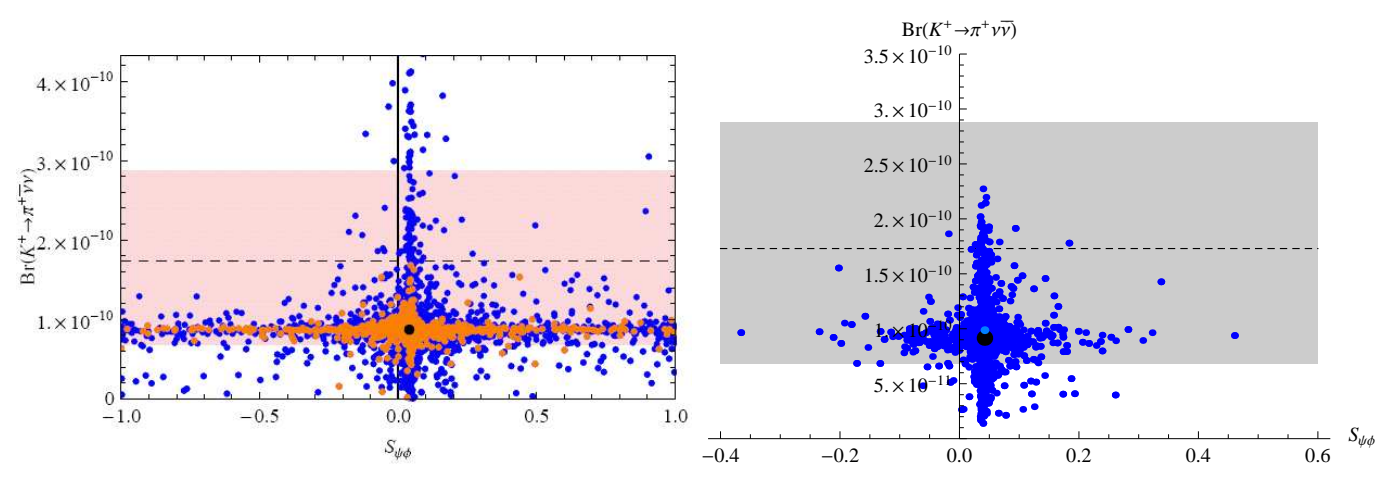

Figure 7: $\operatorname{Br}\left(K^{+} \rightarrow \pi^{+} v \bar{v}\right)$ vs. $S_{\psi \phi}$ in the RSc model (left) [83] and in the LHT model (right) [73].

On the other hand $S_{\psi \phi}$ in the flavour models with only left-handed currents and in the FBMSSM is SM-like.

Clearly a sizable $S_{\psi \phi}$ is not the only manifestation of CP violation in the $B_{s}$ system but presently it is the most prominent one as it can be measured accurately at $\mathrm{LHCb}$, it is theoretically rather clean and the leftover uncertainties could be further decreased using the strategies in [291]. In Fig. 6 we show the correlation between the semi-leptonic asymmetry $A_{S L}^{S}$ and $S_{\psi \phi}$ in the RSc and AC models. This correlation is basically model independent [296] and shows that in any model in which $S_{\psi \phi}$ deviates significantly from its SM value, also $A_{S L}^{s}$ will be very much enhanced. Other implications of a large $S_{\psi \phi}$ in the context of concrete models will be discussed below.

\subsection{Correlations between $\mathbf{K}^{+} \rightarrow \pi^{+} v \bar{v}, \mathbf{K}_{\mathbf{L}} \rightarrow \pi^{0} v \bar{v}, \mathbf{B}_{\mathbf{s}} \rightarrow \mu^{+} \mu^{-}$and $\mathbf{S}_{\psi \phi}$}

In Table 1 we collect the largest possible enhancements for the corresponding branching ratios and $S_{\psi \phi}$ in various extensions of the SM discussed in this talk. It is evident that if we knew already the values of these four observables that are given to us by nature, we could already make a clear distinction between certain scenarios provided the deviations from the SM would be large.

This table does not take into account possible correlations between these four observables and it is important to list some of them:

- Simultaneous enhancements of $S_{\psi \phi}$ and of $\operatorname{Br}(K \rightarrow \pi v \bar{v})$ in the LHT and the RSc scenario are rather unlikely $[73,83]$. This feature is more pronounced in the RSc model. We show this correlation in Fig. 7.

- On the contrary the desire to explain the $S_{\psi \phi}$ anomaly within the supersymmetric flavour models with right-handed currents implies, in the case of the AC and AKM models, values of $\operatorname{Br}\left(B_{s} \rightarrow \mu^{+} \mu^{-}\right)$as high as several $10^{-8}$. This are very exciting news for the CDF, D0 and LHCb experiments! In the RVV2 model such values are also possible but not necessarily implied by the large value of $S_{\psi \phi}$. We show one example of this spectacular correlation for the case of the AC model in the left plot of Fig. 8.

- While in the case of the LHT model some definite correlations between $\operatorname{Br}\left(K_{L} \rightarrow \pi^{0} v \bar{v}\right)$ and $\operatorname{Br}\left(K^{+} \rightarrow \pi^{+} v \bar{v}\right)$ can be seen [73], no such correlations are found in the case of the 
RSc model [83], although in both models the enhancements of the two branching ratios can take place simultaneously. We show this feature in Fig. 9. Some insights in this different behaviour have been recently provided in [297].

More correlations in all these models can be found in the papers quoted above but I think the first two on the list above are the most interesting in the quark flavour sector. Certainly a precise measurement of $S_{\psi \phi}$, in particular if $S_{\psi \phi}$ will be found to be much larger than its SM value, will have an important impact on the models discussed here.
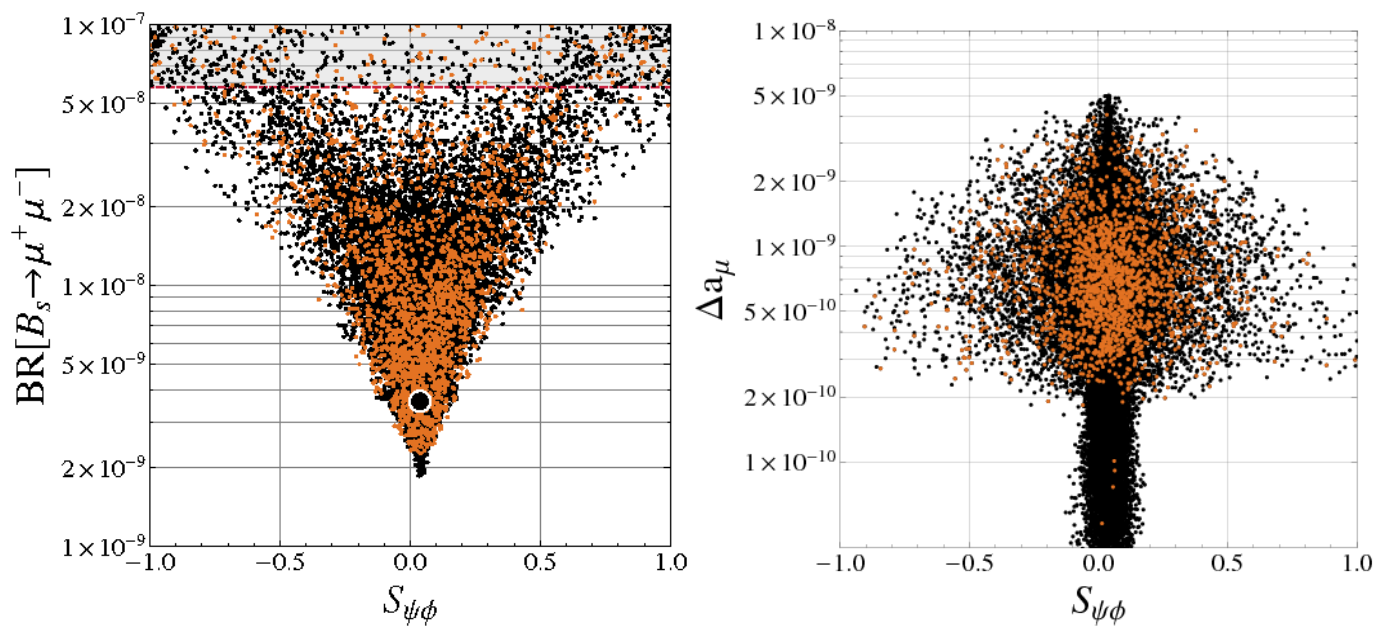

Figure 8: $\operatorname{Br}\left(B_{s} \rightarrow \mu^{+} \mu^{-}\right)$vs. $S_{\psi \phi}$ (left) and $\Delta a_{\mu}$ vs. $S_{\psi \phi}$ (right) in the AC model as obtained in [55].

\subsection{The Correlation between the $S_{\psi \phi}$ and $S_{\phi K_{S}}$ Anomalies}

Before leaving quark flavour physics let me return for a moment to the $S_{\phi K_{S}}$ anomaly in (3.7) and discuss it together with the $S_{\psi \phi}$ anomaly. These anomalies can be explained simultaneously in the GMSSM but the situation is more interesting in supersymmetric flavour (SF) models.

Indeed the SUSY flavour models with right-handed currents (AC, RVV2, AKM) and those with exclusively left-handed currents $(\delta L L)$ can be globally distinguished by the values of the CPasymmetries $S_{\psi \phi}$ and $S_{\phi K_{S}}$ with the following important result: none of the models considered by us in [55] can simultaneously explain the $S_{\psi \phi}$ and $S_{\phi K_{S}}$ anomalies observed in the data. In the models with right-handed currents, $S_{\psi \phi}$ can naturally be much larger than its SM value, while $S_{\phi K_{S}}$ remains either SM-like or its correlation with $S_{\psi \phi}$ is inconsistent with the data. On the contrary, in the models with left-handed currents only, $S_{\psi \phi}$ remains SM-like, while the $S_{\phi K_{S}}$ anomaly can easily be solved. Thus already precise measurements of $S_{\psi \phi}$ and $S_{\phi K_{S}}$ in the near future will select one of these two classes of models, if any.

We will still have something to say about the correlation of these two anomalies with observables in the lepton sector in the context of the SF models in question.

\subsection{Lepton Flavour Violation, EDM's and $(\mathrm{g}-2)_{\mu}$}

Let us finally discuss some additional aspects of Goals 16-18 on our list for the next decade. In [55] we have also performed a very detailed analysis of LFV, EDM's and of $(g-2)_{\mu}$ in the 

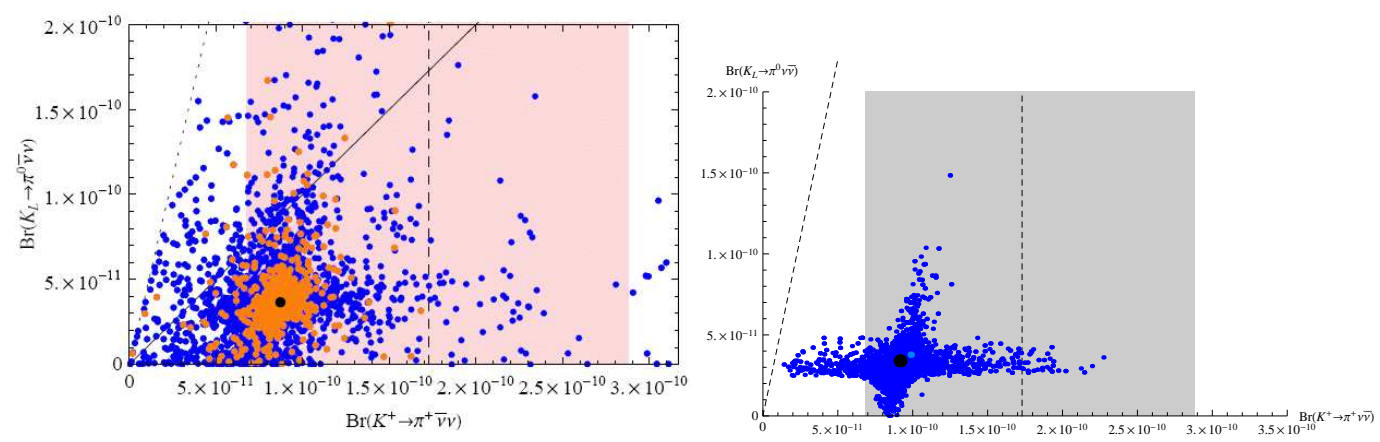

Figure 9: $\operatorname{Br}\left(K_{L} \rightarrow \pi^{0} v \bar{v}\right)$ vs. $\operatorname{Br}\left(K^{+} \rightarrow \pi^{+} v \bar{v}\right)$ in the RSc model (left) [83] and in the LHT model (right) [73].

supersymmetric flavour models AC, RVV2, AKM and $\delta$ LL. Particular emphasis has been put on correlations between these observables in each of these models and their correlation with flavour observables in the quark sector discussed exclusively in this section until now. Let us just list the most striking results of this study keeping in mind that the models with right-handed currents (AC, $\mathrm{RVV} 2, \mathrm{AKM}$ ) have the potential to explain the $S_{\psi \phi}$ anomaly while the $\delta$ LL model could explain the $S_{\phi K_{S}}$ anomaly. Here we go.

1. The desire to explain the $S_{\psi \phi}$ anomaly within the models with right-handed currents automatically implies a solution to the $(g-2)_{\mu}$ anomaly. We illustrate this for the AC model in the right plot of Fig. 8 .

2. In the RVV2 and the AKM models, a large value of $S_{\psi \phi}$ combined with the desire to explain the $(g-2) \mu$ anomaly implies $\operatorname{Br}(\mu \rightarrow e \gamma)$ in the reach of the MEG experiment. In the case of the RVV2 model, $d_{e} \geq 10^{-29} \mathrm{e} \mathrm{cm}$. is predicted, while in the AKM model it is typically smaller. Moreover, in the case of the RVV2 model, $\operatorname{Br}(\tau \rightarrow \mu \gamma) \geq 10^{-9}$ is then in the reach of Super-B machines, while this is not the case in the AKM model. Some of these results are illustrated in Fig. 10.

3. The hadronic EDM's represent very sensitive probes of SUSY flavour models with righthanded currents. In the AC model, large values for the neutron EDM might be easily generated by both the up- and strange-quark (C)EDM. In the former case, visible CP-violating effects in $D^{0}-\bar{D}^{0}$ mixing are also expected while in the latter case large CP-violating effects in the $B_{s}$ system are unavoidable. The RVV2 and AKM models predict values for the down-quark (C)EDM and, hence for the neutron EDM, above the $\approx 10^{-28} e \mathrm{~cm}$. level if a large $S_{\psi \phi}$ is generated. All the above models predict a large strange-quark (C)EDM, hence, a reliable knowledge of its contribution to the hadronic EDM's by means of lattice QCD techniques would be of the utmost importance to probe or to falsify flavour models embedded in a SUSY framework.

4. In the supersymmetric models with exclusively left-handed currents $(\delta \mathrm{LL})$, the desire to explain the $S_{\phi K_{S}}$ anomaly implies automatically a solution to the $(g-2)_{\mu}$ anomaly and the direct $\mathrm{CP}$ asymmetry in $b \rightarrow s \gamma$ much larger than its SM value. We illustrate this in Fig. 11. Similar results are found in the FBMSSM [50]. This is in contrast to the models with right-handed currents where the $A_{\mathrm{CP}}^{b s \gamma}$ asymmetry remains SM-like. 

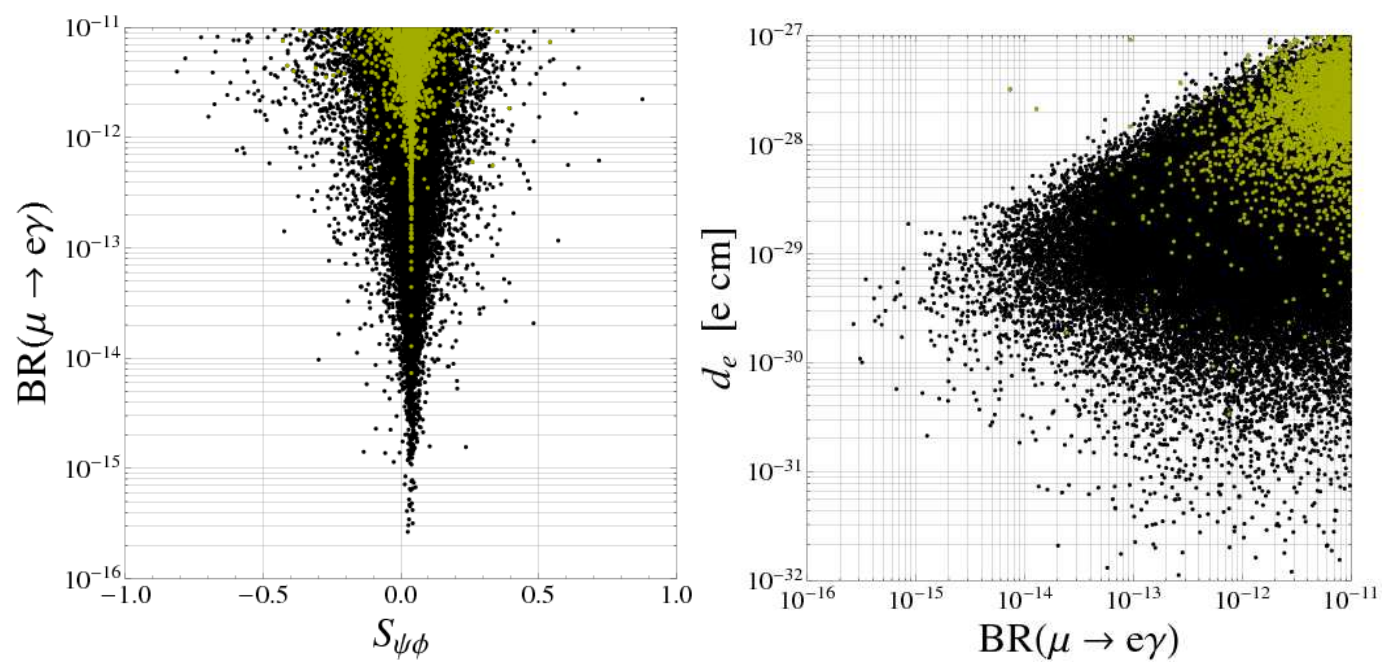

Figure 10: $\operatorname{Br}(\mu \rightarrow e \gamma)$ vs. $S_{\psi \phi}$ (left) and $d_{e}$ vs. $\operatorname{Br}(\mu \rightarrow e \gamma)$ (right) in the RVV2 model as obtained in [55]. The green points explain the $(g-2) \mu$ anomaly at $95 \%$ C.L., i.e. $\Delta a_{\mu} \geq 1 \times 10^{-9}$.

\subsection{Testing GUT Models with Rare B Decays}

Next we would like to stress the power of the complex of rare $B$ decays $B \rightarrow X_{s} \gamma, B \rightarrow X_{s} l^{+} l^{-}$, $B_{s, d} \rightarrow \mu^{+} \mu^{-}$and $B^{+} \rightarrow \tau^{+} v_{\tau}$ in testing NP models. Many analyses of this type can be found in the literature. Here I would like to mention only the analysis of a very interesting $S O(10)$-GUT model of Dermisek and Raby [298] which gives a successful description of quark and lepton masses, of the PMNS matrix and of all elements of the CKM matrix except possibly for $\left|V_{u b}\right|$ that is found to be $3.2 \cdot 10^{-3}$, definitely a bit too low. Yet as shown in [299], this model fails to describe simultaneously the data on the rare decays in question with supersymmetric particles in the reach of the LHC. This is mainly due to $\tan \beta=50$ required in this model. It can be shown that this is a problem of most GUTs with Yukawa unification [300]. Possible solutions to this problem have been suggested in that paper. This discussion demonstrates that flavour physics can have a significant impact not only on physics at the LHC scales but also indirectly for much shorter scales connected with GUT's.

\subsection{A DNA-Flavour Test of New Physics Models}

We have seen above that the patterns of flavour violation found in various extensions of the SM differed from model to model, thereby allowing in the future to find out which of the models discussed by us, if any, can survive the future measurements. Undoubtedly, the correlations between various observables that are often characteristic for a given model will be of the utmost importance in these tests.

In Table 2, taken from [55], a summary of the potential size of deviations from the SM results allowed for a large number of observables, considered in that paper and here, has been presented, taking into account all existing constraints from other observables. This table can be considered as the collection of the DNA's for various models. These DNA's will be modified as new experimental data will be availabe and in certain cases we will be able to declare certain models to be disfavoured or even ruled out. It should be emphasized that in constructing the table we did not take into 
account possible correlations among the observables listed there. We have seen that in some models it is not possible to obtain large effects simultaneously for certain pairs or sets of observables and consequently future measurements of a few observables considered in that table will have an impact on the patterns shown there. It will be interesting to monitor the changes in this table when future experiments will provide new results.
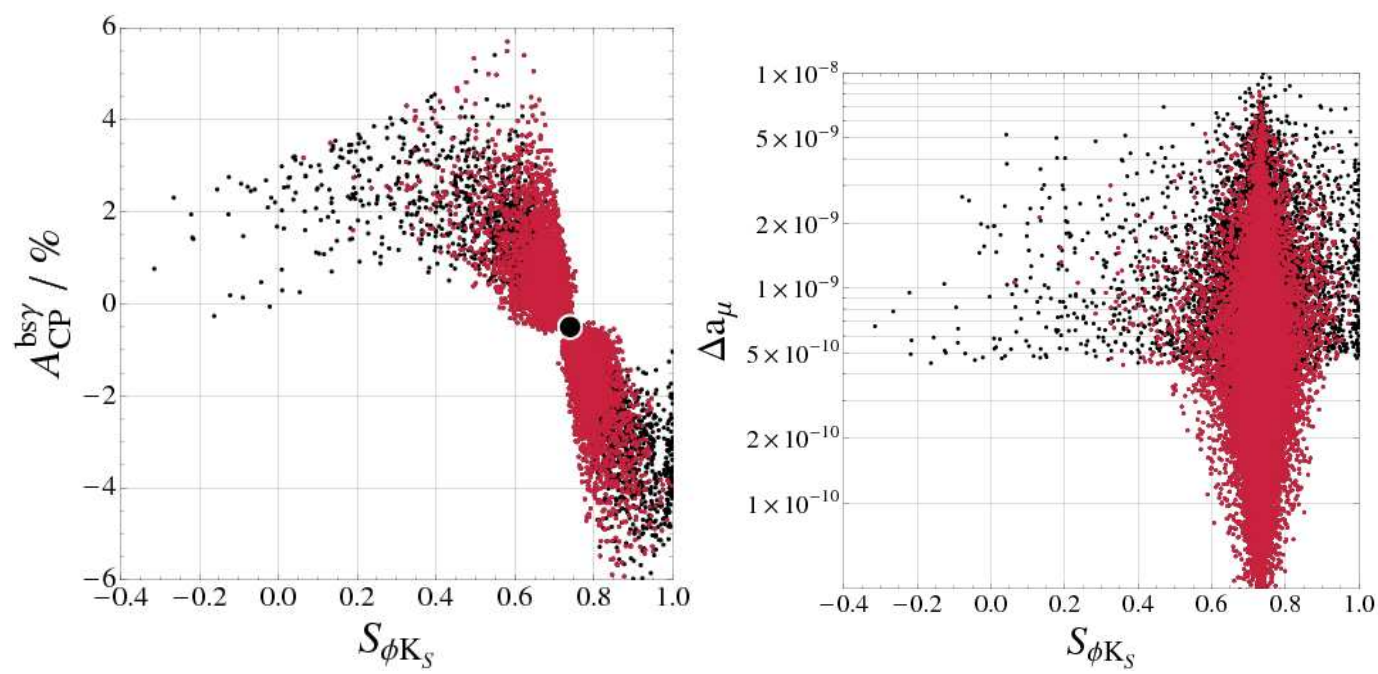

Figure 11: $A_{\mathrm{CP}}^{b s \gamma}$ vs. $S_{\phi K_{S}}$ (left) and $\Delta a_{\mu}$ vs. $S_{\phi K_{S}}$ (right) in the $\delta$ LL model as obtained in [55]. The red points satisfy $\operatorname{Br}\left(B_{s} \rightarrow \mu^{+} \mu^{-}\right) \leq 6 \times 10^{-9}$.

\section{Final Messages and Five Big Questions}

In our search for a more fundamental theory we need to improve our understanding of flavour physics. The study of flavour physics in conjuction with direct collider searches for new physics, with electroweak precision tests and cosmological investigations will without any doubt lead one day to a NSM. Whether this will happen in 2026 or only in 2046 it is not clear at present. Afterall, 35 years have passed since the completion of the present SM and no fully convincing candidate for the NSM exists in the literature. On the other hand in view of presently running and upcoming experiments, the next decade could be like 1970's in which practically every year a new important discovery has been made. Even if by 2026 a NSM may not exist yet, it is conceivable that we will be able to answer the following crucial questions by then:

- Are there any fundamental scalars with masses $M_{s} \leq 1 \mathrm{TeV}$ ?

- Are there any new fundamental fermions like vector-like fermions or the 4th generation of quarks and leptons?

- Are there any new gauge bosons leading to new forces at very short distance scales and an extended gauge group?

- What are the precise patterns of interactions between the gauge bosons, fermions and scalars with respect to flavour and CP Violation? 


\begin{tabular}{|l|c|c|c|c|c|c|c|}
\hline & AC & RVV2 & AKM & $\delta$ LL & FBMSSM & LHT & RS \\
\hline \hline$D^{0}-\bar{D}^{0}$ & $\star \star \star$ & $\star$ & $\star$ & $\star$ & $\star$ & $\star \star \star$ & $?$ \\
\hline$\varepsilon_{K}$ & $\star$ & $\star \star \star$ & $\star \star \star$ & $\star$ & $\star$ & $\star \star$ & $\star \star \star$ \\
\hline$S_{\psi \phi}$ & $\star \star \star$ & $\star \star \star$ & $\star \star \star$ & $\star$ & $\star$ & $\star \star \star$ & $\star \star \star$ \\
\hline \hline$S_{\phi K_{S}}$ & $\star \star \star$ & $\star \star$ & $\star$ & $\star \star \star$ & $\star \star \star$ & $\star$ & $?$ \\
\hline$A_{\mathrm{CP}}\left(B \rightarrow X_{s} \gamma\right)$ & $\star$ & $\star$ & $\star$ & $\star \star \star$ & $\star \star \star$ & $\star$ & $?$ \\
\hline$A_{7,8}\left(B \rightarrow K^{*} \mu^{+} \mu^{-}\right)$ & $\star$ & $\star$ & $\star$ & $\star \star \star$ & $\star \star \star$ & $\star \star$ & $?$ \\
\hline$A_{9}\left(B \rightarrow K^{*} \mu^{+} \mu^{-}\right)$ & $\star$ & $\star$ & $\star$ & $\star$ & $\star$ & $\star$ & $?$ \\
\hline$B \rightarrow K^{(*)} \nu \bar{v}$ & $\star$ & $\star$ & $\star$ & $\star$ & $\star$ & $\star$ & $\star$ \\
\hline$B_{s} \rightarrow \mu^{+} \mu^{-}$ & $\star \star \star$ & $\star \star \star$ & $\star \star \star$ & $\star \star \star$ & $\star \star \star$ & $\star$ & $\star$ \\
\hline$K^{+} \rightarrow \pi^{+} v \bar{v}$ & $\star$ & $\star$ & $\star$ & $\star$ & $\star$ & $\star \star \star$ & $\star \star \star$ \\
\hline$K_{L} \rightarrow \pi^{0} v \bar{v}$ & $\star$ & $\star$ & $\star$ & $\star$ & $\star$ & $\star \star \star$ & $\star \star \star$ \\
\hline$\mu \rightarrow e \gamma$ & $\star \star \star$ & $\star \star \star$ & $\star \star \star$ & $\star \star \star$ & $\star \star \star$ & $\star \star \star$ & $\star \star \star$ \\
\hline \hline$d_{n}$ & $\star \star \star$ & $\star \star \star$ & $\star \star \star$ & $\star \star$ & $\star \star \star$ & $\star$ & $\star \star \star$ \\
\hline$d_{e}$ & $\star \star \star$ & $\star \star \star$ & $\star \star$ & $\star$ & $\star \star \star$ & $\star$ & $\star \star \star$ \\
\hline$(g-2)_{\mu}$ & $\star \star \star$ & $\star \star \star$ & $\star \star$ & $\star \star \star$ & $\star \star \star$ & $\star$ & $\star \star$ \\
\hline
\end{tabular}

Table 2: "DNA" of flavour physics effects [55] for the most interesting observables in a selection of SUSY and non-SUSY models. $\star \star \star \star$ signals large effects, $\star \star$ visible but small effects and $\star$ implies that the given model does not predict sizable effects in that observable.

- Can the answers to these four questions help us in understanding the BAU and other fundamental cosmological questions?

There are of course many other profound questions [301] related to grand unification, gravity and string theory and to other aspects of elementary particle physics and cosmology but from my point of view I would really be happy if in 2026 satisfactory answers to the five questions posed above were available.

In this review written at the advent of the LHC era to which also several low energy precision machines belong, I wanted to emphasize that many observables in the quark and lepton flavour sectors have not been measured yet or are only poorly known and that flavour physics only now enters the precision era. Indeed, spectacular deviations from the SM and MFV expectations are still possible in flavour physics. The interplay of the expected deviations with direct searches at Tevatron, LHC and later at ILC will be most interesting.

In particular I emphasized the role of correlations between various observables in our search 
for the fundamental theory of flavour. These correlations and hopefully new discoveries, both in flavour physics and in direct searches for NP will pave the road to the New Standard Model.

Acknowledgments I would like to thank the organizers of EPS09 for inviting me to give this talk at such a well organized and interesting conference. I would like to thank all my collaborators for a wonderful time we spent together exploring different avenues beyond the Standard Model. Special thanks go to Björn Duling for invaluable comments on the manuscript and to Wolfgang Altmannshofer and Monika Blanke for helping me at various stages of this writeup. This research was partially supported by the Deutsche Forschungsgemeinschaft (DFG) under contract BU 706/2-1, the DFG Cluster of Excellence 'Origin and Structure of the Universe' and by the German Bundesministerium für Bildung und Forschung under contract 05HT6WOA.

\section{References}

[1] W. Heisenberg, Über den anschaulichen Inhalt der quantentheoretischen Kinematik und Mechanik, Z. Phys. 43 (1927) 172-198, [physics / 0605038 ].

[2] N. Cabibbo, Unitary Symmetry and Leptonic Decays, Phys. Rev. Lett. 10 (1963) 531-533.

[3] M. Kobayashi and T. Maskawa, CP Violation in the Renormalizable Theory of Weak Interaction, Prog. Theor. Phys. 49 (1973) 652-657.

[4] B. Pontecorvo, Inverse beta processes and nonconservation of lepton charge, Sov. Phys. JETP 7 (1958) 172-173.

[5] Z. Maki, M. Nakagawa, and S. Sakata, Remarks on the unified model of elementary particles, Prog. Theor. Phys. 28 (1962) 870-880.

[6] A. J. Bevan, Flavour Physics at B-factories and other machines, arXiv:0909.2519.

[7] M. Artuso et. al., B, D and K decays, Eur. Phys. J. C57 (2008) 309-492, [arXiv: 0801 . 1833].

[8] M. Raidal et. al., Flavour physics of leptons and dipole moments, Eur. Phys. J. C57 (2008) 13-182, [arXiv:0801.1826].

[9] M. Antonelli et. al., Flavor Physics in the Quark Sector, arXiv:0907.5386.

[10] M. Bona et. al., SuperB: A High-Luminosity Asymmetric $e^{+} e^{-}$Super Flavor Factory. Conceptual Design Report, arXiv:0709.0451.

[11] T. E. Browder, T. Gershon, D. Pirjol, A. Soni, and J. Zupan, New Physics at a Super Flavor Factory, arXiv:0802.3201.

[12] G. C. Branco, L. Lavoura, and J. P. Silva, CP violation, Int. Ser. Monogr. Phys. 103 (1999) 1-536.

[13] I. I. Y. Bigi and A. I. Sanda, CP violation, Camb. Monogr. Part. Phys. Nucl. Phys. Cosmol. 9 (2000) $1-382$.

[14] G. Buchalla, A. J. Buras, and M. E. Lautenbacher, Weak Decays Beyond Leading Logarithms, Rev. Mod. Phys. 68 (1996) 1125-1144, [hep-ph/9512380].

[15] A. J. Buras, Weak Hamiltonian, CP violation and rare decays, hep-ph/9806471.

[16] A. J. Buras, Flavor dynamics: CP violation and rare decays, hep-ph/0101336. 
[17] A. J. Buras, P. Gambino, M. Gorbahn, S. Jager, and L. Silvestrini, Universal unitarity triangle and physics beyond the standard model, Phys. Lett. B500 (2001) 161-167, [hep-ph/ 0007085 ].

[18] A. J. Buras, Minimal flavor violation, Acta Phys. Polon. B34 (2003) 5615-5668, [hep-ph/0310208].

[19] M. Blanke, A. J. Buras, D. Guadagnoli, and C. Tarantino, Minimal Flavour Violation Waiting for Precise Measurements of $\Delta M_{s}, S_{\psi \phi}, A_{\mathrm{SL}}^{s},\left|V_{u b}\right|, \gamma$ and $B_{s, d}^{0} \rightarrow \mu^{+} \mu^{-}, J H E P 10$ (2006) 003, [hep-ph/0604057].

[20] A. J. Buras, Testing the CKM Picture of Flavour and CP Violation in Rare K and B Decays and Particle-Antiparticle Mixing, arXiv:0904.4917.

[21] G. D’Ambrosio, G. F. Giudice, G. Isidori, and A. Strumia, Minimal flavour violation: An effective field theory approach, Nucl. Phys. B645 (2002) 155-187, [hep-ph/ 0207036$].$

[22] R. S. Chivukula and H. Georgi, Composite Technicolor Standard Model, Phys. Lett. B188 (1987) 99.

[23] L. J. Hall and L. Randall, Weak scale effective supersymmetry, Phys. Rev. Lett. 65 (1990) 2939-2942.

[24] M. Beneke, G. Buchalla, C. Greub, A. Lenz, and U. Nierste, Next-to-leading order QCD corrections to the lifetime difference of $B_{s}$ mesons, Phys. Lett. B459 (1999) 631-640, [hep-ph/9808385].

[25] M. Beneke, G. Buchalla, C. Greub, A. Lenz, and U. Nierste, The $B^{+}-B_{d}^{0}$ lifetime difference beyond leading logarithms, Nucl. Phys. B639 (2002) 389-407, [hep-ph / 0202106 ].

[26] M. Ciuchini, E. Franco, V. Lubicz, F. Mescia, and C. Tarantino, Lifetime differences and CP violation parameters of neutral B mesons at the next-to-leading order in QCD, JHEP 08 (2003) 031, [hep-ph/0308029].

[27] H. H. Asatryan, H. M. Asatrian, C. Greub, and M. Walker, Complete gluon bremsstrahlung corrections to the process $b \rightarrow s l^{+} l^{-}$, Phys. Rev. D66 (2002) 034009, [hep-ph/ 0204341$]$.

[28] H. M. Asatrian, K. Bieri, C. Greub, and A. Hovhannisyan, NNLL corrections to the angular distribution and to the forward-backward asymmetries in $b \rightarrow X_{s} l^{+} l^{-}$, Phys. Rev. D66 (2002) 094013, [hep-ph/0209006].

[29] P. Gambino, M. Gorbahn, and U. Haisch, Anomalous Dimension Matrix for Radiative and Rare Semileptonic B Decays up to Three Loops, Nucl. Phys. B673 (2003) 238-262, [hep-ph/0306079].

[30] A. Ghinculov, T. Hurth, G. Isidori, and Y. P. Yao, The rare decay $B \rightarrow X_{s} l^{+} l^{-}$to NNLL precision for arbitrary dilepton invariant mass, Nucl. Phys. $\mathbf{B 6 8 5}$ (2004) 351-392, [hep-ph/0312128].

[31] A. Ghinculov, T. Hurth, G. Isidori, and Y. P. Yao, Forward-backward asymmetry in $B \rightarrow X_{s} l^{+} l^{-}$at the NNLL level, Nucl. Phys. B648 (2003) 254-276, [hep-ph / 0208088$].$

[32] C. Bobeth, P. Gambino, M. Gorbahn, and U. Haisch, Complete NNLO QCD analysis of $\bar{B} \rightarrow X_{s} l^{+} l^{-}$ and higher order electroweak effects, JHEP 04 (2004) 071, [hep-ph / 0312090$].$

[33] M. Beneke, T. Feldmann, and D. Seidel, Exclusive radiative and electroweak $b \rightarrow d$ and $b \rightarrow s$ penguin decays at NLO, Eur. Phys. J. C41 (2005) 173-188, [hep-ph/ 0412400 ].

[34] A. J. Buras, M. Gorbahn, U. Haisch, and U. Nierste, The rare decay $K^{+} \rightarrow \pi^{+} v \bar{v}$ at the next-to-nextto-leading order in QCD, Phys. Rev. Lett. 95 (2005) 261805, [hep-ph / 0508165$].$

[35] A. J. Buras, M. Gorbahn, U. Haisch, and U. Nierste, Charm quark contribution to $K^{+} \rightarrow \pi^{+} v \bar{v}$ at next-to-next-to-leading order, JHEP 11 (2006) 002, [hep-ph / 0603079 ]. 
[36] J. Brod and M. Gorbahn, Electroweak Corrections to the Charm Quark Contribution to $K^{+} \rightarrow \pi^{+} v \bar{v}$, Phys. Rev. D78 (2008) 034006, [arXiv: 0805.4119].

[37] M. Misiak et. al., The first estimate of $B\left(\bar{B} \rightarrow X_{s} \gamma\right)$ at $O\left(\alpha_{s}^{2}\right)$, Phys. Rev. Lett. 98 (2007) 022002, [hep-ph/0609232].

[38] M. Gorbahn and U. Haisch, Effective Hamiltonian for non-leptonic $|\Delta(F)|=1$ decays at NNLO in QCD, Nucl. Phys. B713 (2005) 291-332, [hep-ph / 0411071$].$

[39] T. Feldmann and T. Mannel, Minimal Flavour Violation and Beyond, JHEP 02 (2007) 067, [hep-ph/0611095].

[40] G. Colangelo, E. Nikolidakis, and C. Smith, Supersymmetric models with minimal flavour violation and their running, Eur. Phys. J. C59 (2009) 75-98, [arXiv: 0807 . 0801].

[41] P. Paradisi, M. Ratz, R. Schieren, and C. Simonetto, Running minimal flavor violation, Phys. Lett. B668 (2008) 202-209, [arXiv : 0805 . 3989].

[42] L. Mercolli and C. Smith, EDM constraints on flavored CP-violating phases, Nucl. Phys. $\mathbf{B 8 1 7}$ (2009) 1-24, [arXiv:0902.1949].

[43] T. Feldmann, M. Jung, and T. Mannel, Sequential Flavour Symmetry Breaking, Phys. Rev. D80 (2009) 033003, [arXiv:0906.1523].

[44] A. L. Kagan, G. Perez, T. Volansky, and J. Zupan, General Minimal Flavor Violation, arXiv:0903.1794.

[45] P. Paradisi and D. Straub, The SUSY CP Problem and the MFV Principle, arXiv : 0906.4551.

[46] S. Baek and P. Ko, Probing SUSY-induced CP violations at B factories, Phys. Rev. Lett. 83 (1999) 488-491, [hep-ph/9812229].

[47] S. Baek and P. Ko, Effects of supersymmetric CP violating phases on $B \rightarrow X_{S} l^{+} l^{-}$and $\varepsilon_{K}$, Phys. Lett. B462 (1999) 95-102, [hep-ph/9904283].

[48] A. Bartl et. al., General flavor blind MSSM and CP violation, Phys. Rev. D64 (2001) 076009, [hep-ph/0103324].

[49] J. R. Ellis, J. S. Lee, and A. Pilaftsis, B-Meson Observables in the Maximally CP-Violating MSSM with Minimal Flavour Violation, Phys. Rev. D76 (2007) 115011, [arXiv: 0708 . 2079].

[50] W. Altmannshofer, A. J. Buras, and P. Paradisi, Low Energy Probes of CP Violation in a Flavor Blind MSSM, Phys. Lett. B669 (2008) 239-245, [arXiv : 0808 . 0707].

[51] C. Bobeth et. al., Upper bounds on rare $K$ and B decays from minimal flavor violation, Nucl. Phys. B726 (2005) 252-274, [hep-ph/0505110].

[52] U. Haisch and A. Weiler, Determining the Sign of the Z-Penguin Amplitude, Phys. Rev. D76 (2007) 074027, [arXiv:0706.2054].

[53] T. Hurth, G. Isidori, J. F. Kamenik, and F. Mescia, Constraints on New Physics in MFV models: a model-independent analysis of $\Delta F=1$ processes, Nucl. Phys. $\mathbf{B 8 0 8}$ (2009) 326-346, [arXiv:0807.5039].

[54] S. P. Martin, A supersymmetry primer, hep-ph/9709356.

[55] W. Altmannshofer, A. J. Buras, S. Gori, P. Paradisi, and D. M. Straub, Anatomy and Phenomenology of FCNC and CPV Effects in SUSY Theories, arXiv: 0909.1333. 
[56] K. Agashe and C. D. Carone, Supersymmetric flavor models and the $B \rightarrow \phi K_{S}$ anomaly, Phys. Rev. D68 (2003) 035017, [hep-ph / 030422 ].

[57] G. G. Ross, L. Velasco-Sevilla, and O. Vives, Spontaneous CP violation and non-Abelian family symmetry in SUSY, Nucl. Phys. B692 (2004) 50-82, [hep-ph/ 0401064 ].

[58] L. Calibbi et. al., FCNC and CP Violation Observables in a SU(3)-flavoured MSSM, arXiv:0907.4069.

[59] S. Antusch, S. F. King, and M. Malinsky, Solving the SUSY Flavour and CP Problems with SU(3) Family Symmetry, JHEP 06 (2008) 068, [arXiv : 0708 . 1282].

[60] L. J. Hall and H. Murayama, A Geometry of the generations, Phys. Rev. Lett. 75 (1995) 3985-3988, [hep-ph/9508296].

[61] W. Altmannshofer, Probing the MSSM flavor structure with low energy CP violation, arXiv:0909.2837.

[62] A. J. Buras, Patterns of Flavour Violation in the RSc Model, the LHT Model and Supersymmetric Flavour Models, arXiv:0909.3206.

[63] L. J. Hall, V. A. Kostelecky, and S. Raby, New Flavor Violations in Supergravity Models, Nucl. Phys. B267 (1986) 415.

[64] F. Gabbiani, E. Gabrielli, A. Masiero, and L. Silvestrini, A complete analysis of FCNC and CP constraints in general SUSY extensions of the standard model, Nucl. Phys. B477 (1996) 321-352, [hep-ph/9604387].

[65] A. Dedes, J. Rosiek, and P. Tanedo, Complete One-Loop MSSM Predictions for $B^{0} \rightarrow \ell^{+} \ell^{\prime-}$ at the Tevatron and LHC, Phys. Rev. D79 (2009) 055006, [arXiv: 0812 . 4320].

[66] M. Schmaltz and D. Tucker-Smith, Little Higgs review, Ann. Rev. Nucl. Part. Sci. 55 (2005) 229-270, [hep-ph/0502182].

[67] M. Perelstein, Little Higgs models and their phenomenology, Prog. Part. Nucl. Phys. 58 (2007) 247-291, [hep-ph/0512128].

[68] N. Arkani-Hamed, A. G. Cohen, E. Katz, and A. E. Nelson, The littlest Higgs, JHEP 07 (2002) 034, [hep-ph/0206021].

[69] H.-C. Cheng and I. Low, TeV symmetry and the little hierarchy problem, JHEP 09 (2003) 051 , [hep-ph/0308199].

[70] H.-C. Cheng and I. Low, Little hierarchy, little Higgses, and a little symmetry, JHEP 08 (2004) 061, [hep-ph/ 0405243$]$.

[71] M. Blanke and A. J. Buras, A guide to flavour changing neutral currents in the littlest Higgs model with T-parity, Acta Phys. Polon. B38 (2007) 2923, [hep-ph / 0703117$].$

[72] B. Duling, Lepton Flavor Violation in the LHT - A Clear Distinction from Supersymmetry, arXiv:0709.4413.

[73] M. Blanke, A. J. Buras, B. Duling, S. Recksiegel, and C. Tarantino, FCNC Processes in the Littlest Higgs Model with T-Parity: a 2009 Look, arXiv: 0906.5454.

[74] L. Randall and R. Sundrum, A large mass hierarchy from a small extra dimension, Phys. Rev. Lett. 83 (1999) 3370-3373, [hep-ph/9905221]. 
[75] T. Gherghetta and A. Pomarol, Bulk fields and supersymmetry in a slice of AdS, Nucl. Phys. B586 (2000) 141-162, [hep-ph/0003129].

[76] S. Chang, J. Hisano, H. Nakano, N. Okada, and M. Yamaguchi, Bulk standard model in the Randall-Sundrum background, Phys. Rev. D62 (2000) 084025, [hep-ph/99124 98].

[77] Y. Grossman and M. Neubert, Neutrino masses and mixings in non-factorizable geometry, Phys. Lett. B474 (2000) 361-371, [hep-ph/9912408].

[78] G. Burdman, Flavor violation in warped extra dimensions and CP asymmetries in B decays, Phys. Lett. B590 (2004) 86-94, [hep-ph/ 0310144 ].

[79] S. J. Huber, Flavor violation and warped geometry, Nucl. Phys. B666 (2003) 269-288, [hep-ph/0303183].

[80] K. Agashe, G. Perez, and A. Soni, Flavor structure of warped extra dimension models, Phys. Rev. D71 (2005) 016002, [hep-ph / 0408134$].$

[81] C. Csaki, A. Falkowski, and A. Weiler, The Flavor of the Composite Pseudo-Goldstone Higgs, JHEP 09 (2008) 008, [arXiv: 0804 . 1954].

[82] M. Blanke, A. J. Buras, B. Duling, S. Gori, and A. Weiler, $\Delta F=2$ Observables and Fine-Tuning in a Warped Extra Dimension with Custodial Protection, JHEP 03 (2009) 001, [arXiv: 0809.1073 ].

[83] M. Blanke, A. J. Buras, B. Duling, K. Gemmler, and S. Gori, Rare K and B Decays in a Warped Extra Dimension with Custodial Protection, JHEP 03 (2009) 108, [arXiv: 0812 . 3803].

[84] A. J. Buras, B. Duling, and S. Gori, The Impact of Kaluza-Klein Fermions on Standard Model Fermion Couplings in a RS Model with Custodial Protection, JHEP 09 (2009) 076, [arXiv:0905.2318].

[85] B. Duling, $K$ and B meson mixing in warped extra dimensions with custodial protection, J. Phys. Conf. Ser. 171 (2009) 012061, [arXiv : 0901 . 4599].

[86] S. Gori, Patterns of Flavour Violation in a Warped Extra Dimensional Model with Custodial Protection, J. Phys. Conf. Ser. 171 (2009) 012062, [arXiv: 0901.4704 ].

[87] M. Blanke, K and B Physics in the Custodially Protected Randall-Sundrum Model, arXiv:0908.2716.

[88] B. Duling, Predictions for Flavour Observables in a RS Model with Custodial Symmetry, arXiv:0908.3099.

[89] S. Gori, Patterns of Flavor Violation in a Warped Extra Dimensional Model with Custodial Protection, arXiv:0909.3042.

[90] C. Csaki, A. Falkowski, and A. Weiler, A Simple Flavor Protection for RS, Phys. Rev. D80 (2009) 016001, [arXiv:0806.3757].

[91] G. Cacciapaglia et. al., A GIM Mechanism from Extra Dimensions, JHEP 04 (2008) 006, [arXiv:0709.1714].

[92] C. Cheung, A. L. Fitzpatrick, and L. Randall, Sequestering CP Violation and GIM-Violation with Warped Extra Dimensions, JHEP 01 (2008) 069, [arXiv : 0711 . 4421].

[93] J. Santiago, Minimal Flavor Protection: A New Flavor Paradigm in Warped Models, JHEP 12 (2008) 046, [arXiv: 0806.1230$].$ 
[94] C. Csaki and D. Curtin, A Flavor Protection for Warped Higgsless Models, Phys. Rev. D80 (2009) 015027, [arXiv:0904.2137].

[95] C. Csaki, G. Perez, Z. Surujon, and A. Weiler, Flavor Alignment via Shining in RS, arXiv:0907.0474.

[96] K. Agashe, A. Delgado, M. J. May, and R. Sundrum, RS1, custodial isospin and precision tests, JHEP 08 (2003) 050, [hep-ph/ 0308036 ].

[97] C. Csaki, C. Grojean, L. Pilo, and J. Terning, Towards a realistic model of Higgsless electroweak symmetry breaking, Phys. Rev. Lett. 92 (2004) 101802, [hep-ph/0308038].

[98] K. Agashe, R. Contino, L. Da Rold, and A. Pomarol, A custodial symmetry for Zb $\bar{b}$, Phys. Lett. B641 (2006) 62-66, [hep-ph/ 0605341$].$

[99] M. E. Albrecht, M. Blanke, A. J. Buras, B. Duling, and K. Gemmler, Electroweak and Flavour Structure of a Warped Extra Dimension with Custodial Protection, JHEP 09 (2009) 064, [arXiv:0903.2415].

[100] S. Casagrande, F. Goertz, U. Haisch, M. Neubert, and T. Pfoh, Flavor Physics in the Randall-Sundrum Model: I. Theoretical Setup and Electroweak Precision Tests, JHEP 10 (2008) 094, [arXiv: 0807.4937 ].

[101] M. Bauer, S. Casagrande, L. Gruender, U. Haisch, and M. Neubert, Little Randall-Sundrum models: $\varepsilon_{K}$ strikes again, arXiv:0811.3678.

[102] F. del Aguila, M. Perez-Victoria, and J. Santiago, Observable contributions of new exotic quarks to quark mixing, JHEP 09 (2000) 011, [hep-ph / 0007316 ].

[103] G. C. Branco, M. N. Rebelo, and J. I. Silva-Marcos, Yukawa Textures, New Physics and Nondecoupling, Phys. Rev. D76 (2007) 033008, [hep-ph / 0612252$].$

[104] I. Picek and B. Radovcic, Nondecoupling of terascale isosinglet quark and rare $K$ - and B-decays, Phys. Rev. D78 (2008) 015014, [arXiv : 0804 . 2216].

[105] G. W. S. Hou, Search for TeV Scale Physics in Heavy Flavour Decays, Eur. Phys. J. C59 (2009) 521-541, [arXiv:0808.1932].

[106] W.-S. Hou, F.-F. Lee, and C.-Y. Ma, Fourth Generation Leptons and Muon g - 2, Phys. Rev. D79 (2009) 073002, [arXiv:0812.0064].

[107] G. W. S. Hou, Source of CP Violation for the Baryon Asymmetry of the Universe, arXiv:0810.3396.

[108] M. Bobrowski, A. Lenz, J. Riedl, and J. Rohrwild, How much space is left for a new family of fermions?, Phys. Rev. D79 (2009) 113006, [arXiv: 0902 . 4883].

[109] A. Soni, A. K. Alok, A. Giri, R. Mohanta, and S. Nandi, The fourth family: a natural explanation for the observed pattern of anomalies in B-CP asymmetries, arXiv:0807.1971.

[110] G. Eilam, B. Melic, and J. Trampetic, CP violation and the 4th generation, arXiv: 0909.3227.

[111] M. S. Chanowitz, Bounding CKM Mixing with a Fourth Family, Phys. Rev. D79 (2009) 113008, [arXiv:0904.3570].

[112] V. A. Novikov, A. N. Rozanov, and M. I. Vysotsky, Once more on extra quark-lepton generations and precision measurements, arXiv:0904.4570. 
[113] R. Fok and G. D. Kribs, Four Generations, the Electroweak Phase Transition, and Supersymmetry, Phys. Rev. D78 (2008) 075023, [arXiv: 0803 .4207].

[114] G. Burdman, L. Da Rold, O. Eboli, and R. Matheus, A Strongly Coupled Fourth Generation at the LHC, Phys. Rev. D79 (2009) 075026, [arXiv: 0812 . 0368].

[115] T. Appelquist, H.-C. Cheng, and B. A. Dobrescu, Bounds on universal extra dimensions, Phys. Rev. D64 (2001) 035002, [hep-ph / 0012100$].$

[116] W. Altmannshofer, A. J. Buras, D. Guadagnoli, and M. Wick, Large $\left|V_{u b}\right|:$ A challenge for the Minimal Flavour Violating MSSM, JHEP 12 (2007) 096, [arXiv: 0706.3845$].$

[117] A. J. Buras and L. Silvestrini, Upper bounds on $K \rightarrow \pi \nu \bar{v}$ and $K_{L} \rightarrow \pi^{0} e^{+} e^{-}$from $\varepsilon^{\prime} / \varepsilon$ and $K_{L} \rightarrow \mu^{+} \mu^{-}$, Nucl. Phys. B546 (1999) 299-314, [hep-ph/9811471].

[118] A. J. Buras, G. Colangelo, G. Isidori, A. Romanino, and L. Silvestrini, Connections between $\varepsilon^{\prime} / \varepsilon$ and rare kaon decays in supersymmetry, Nucl. Phys. $B 566$ (2000) 3-32, [hep-ph/9908371].

[119] A. J. Buras, R. Fleischer, S. Recksiegel, and F. Schwab, Anatomy of prominent B and K decays and signatures of CP-violating new physics in the electroweak penguin sector, Nucl. Phys. B697 (2004) 133-206, [hep-ph/0402112].

[120] P. Langacker and M. Plumacher, Flavor changing effects in theories with a heavy Z' boson with family non-universal couplings, Phys. Rev. D62 (2000) 013006, [hep-ph/0001204].

[121] V. Barger et. al., $b \rightarrow s$ Transitions in Family-dependent U(1)'Models, arXiv:0906.3745.

[122] P. Langacker, The Physics of New U(1)' Gauge Bosons, arXiv:0909. 3260.

[123] K. Agashe, M. Papucci, G. Perez, and D. Pirjol, Next to minimal flavor violation, hep-ph/0509117.

[124] UTfit Collaboration, M. Bona et. al., Model-independent constraints on $\Delta F=2$ operators and the scale of new physics, JHEP 03 (2008) 049, [arXiv: 0707 . 0636 ].

[125] V. Lubicz and C. Tarantino, Flavour physics and Lattice QCD: averages of lattice inputs for the Unitarity Triangle Analysis, Nuovo Cim. 123B (2008) 674-688, [arXiv:0807.4605].

[126] HPQCD Collaboration, E. Gamiz, C. T. H. Davies, G. P. Lepage, J. Shigemitsu, and M. Wingate, Neutral B Meson Mixing in Unquenched Lattice QCD, Phys. Rev. D80 (2009) 014503, [arXiv:0902.1815].

[127] K. G. Chetyrkin et. al., Charm and Bottom Quark Masses: an Update, arXiv: 0907.2110.

[128] H. Leutwyler, to appear soon.

[129] M. Jamin, J. A. Oller, and A. Pich, Scalar K $\pi$ form factor and light quark masses, Phys. Rev. D74 (2006) 074009, [hep-ph/0605095].

[130] P. A. Baikov, K. G. Chetyrkin, and J. H. Kuhn, Order $\alpha_{s}^{4}$ QCD Corrections to Z and $\tau$ Decays, Phys. Rev. Lett. 101 (2008) 012002, [arXiv:0 801 . 1821].

[131] M. Beneke and M. Jamin, alpha $a_{s}$ and the $\tau$ hadronic width: fixed-order, contour-improved and higher-order perturbation theory, JHEP 09 (2008) 044, [arXiv : 0806 . 3156].

[132] S. Bethke, The 2009 Wolrd Average of $\alpha_{S}\left(M_{Z}\right)$, arXiv:0 908.1135.

[133] Heavy Flavor Averaging Group (HFAG) Collaboration, E. Barberio et. al., Averages of b-hadron properties at the end of 2006, arXiv:0704.3575. Updates available on http://www.slac.stanford.edu/xorg/hfag. 
[134] M. Beneke, G. Buchalla, M. Neubert, and C. T. Sachrajda, QCD factorization for $B \rightarrow \pi \pi$ decays: Strong phases and CP violation in the heavy quark limit, Phys. Rev. Lett. 83 (1999) 1914-1917, [hep-ph/9905312].

[135] M. Beneke, G. Buchalla, M. Neubert, and C. T. Sachrajda, QCD factorization for exclusive, non-leptonic B meson decays: General arguments and the case of heavy-light final states, Nucl. Phys. B591 (2000) 313-418, [hep-ph/0006124].

[136] Y. Y. Keum and A. I. Sanda, Possible large direct CP violations in charmless B decays: Summary report on the p QCD method. ((U)), Phys. Rev. D67 (2003) 054009, [hep-ph/ 0209014$].$

[137] C. W. Bauer, D. Pirjol, and I. W. Stewart, Soft-Collinear Factorization in Effective Field Theory, Phys. Rev. D65 (2002) 054022, [hep-ph/ 0109045$].$

[138] C. W. Bauer, S. Fleming, D. Pirjol, I. Z. Rothstein, and I. W. Stewart, Hard scattering factorization from effective field theory, Phys. Rev. D66 (2002) 014017, [hep-ph / 2202088 ].

[139] C. W. Bauer, D. Pirjol, and I. W. Stewart, Power counting in the soft-collinear effective theory, Phys. Rev. D66 (2002) 054005, [hep-ph / 0205289 ].

[140] M. Beneke and T. Feldmann, Multipole-expanded soft-collinear effective theory with non-abelian gauge symmetry, Phys. Lett. B553 (2003) 267-276, [hep-ph / 211358 ].

[141] M. Beneke, A. P. Chapovsky, M. Diehl, and T. Feldmann, Soft-collinear effective theory and heavy-to-light currents beyond leading power, Nucl. Phys. B643 (2002) 431-476, [hep-ph/0206152].

[142] C.-W. Chiang, M. Gronau, J. L. Rosner, and D. A. Suprun, Charmless B $\rightarrow$ PP decays using flavor SU(3) symmetry, Phys. Rev. D70 (2004) 034020, [hep-ph / 0404073 ].

[143] G. Buchalla, B Physics Theory for Hadron Colliders, arXiv: 0809.0532.

[144] R. Fleischer, Flavour Physics and CP Violation: Expecting the LHC, arXiv: 0802.2882.

[145] L. Silvestrini, Searching for new physics in $b \rightarrow$ s hadronic penguin decays, Ann. Rev. Nucl. Part. Sci. 57 (2007) 405-440, [arXiv: 0705.1624 ].

[146] R. Fleischer and M. Gronau, Studying New Physics Amplitudes in Charmless Bs Decays, Phys. Lett. B660 (2008) 212-216, [arXiv: 0709 . 4013].

[147] S. Baek, C.-W. Chiang, M. Gronau, D. London, and J. L. Rosner, Diagnostic for new physics in $B \rightarrow \pi K$ decays, Phys. Lett. B678 (2009) 97-100, [arXiv: 0905.1495$].$

[148] M. Ciuchini, E. Franco, G. Martinelli, M. Pierini, and L. Silvestrini, Searching For New Physics With B to K pi Decays, Phys. Lett. B674 (2009) 197-203, [arXiv : 0811.0341$].$

[149] R. Fleischer, S. Jager, D. Pirjol, and J. Zupan, Benchmarks for the New-Physics Search through CP Violation in $B^{0} \rightarrow \pi^{0} K_{S}$, Phys. Rev. D78 (2008) 111501, [arXiv: 0806.2900 ].

[150] M. Gronau and J. L. Rosner, Implications for CP asymmetries of improved data on $B \rightarrow K^{0} \pi^{0}$, Phys. Lett. B666 (2008) 467-471, [arXiv: 0807 . 3080].

[151] C. Bobeth, G. Hiller, and G. Piranishvili, CP Asymmetries in $\bar{B} \rightarrow \bar{K}^{*}(\rightarrow \bar{K} p i) l^{+} l^{-}$and Untagged $\bar{B}_{s}, B_{s} \rightarrow \phi\left(\rightarrow K^{+} K^{-}\right) l^{+} l^{-}$Decays at NLO, JHEP 07 (2008) 106, [arXiv: 0805 . 252 5].

[152] U. Egede, T. Hurth, J. Matias, M. Ramon, and W. Reece, New observables in the decay mode $\bar{B} \rightarrow \bar{K}^{0 *} l^{+} l^{-}$, JHEP 11 (2008) 032, [arXiv : 0807.2589$]$. 
[153] W. Altmannshofer et. al., Symmetries and Asymmetries of $B \rightarrow K^{*} \mu^{+} \mu^{-}$Decays in the Standard Model and Beyond, JHEP 01 (2009) 019, [arXiv : 0811 . 1214].

[154] UTfit Collaboration, . M. Bona et. al., An Improved Standard Model Prediction Of BR $(B \rightarrow \tau v)$ And Its Implications For New Physics, arXiv:0908.3470.

[155] BABAR Collaboration, B. Aubert et. al., A Search for $B^{+} \rightarrow \tau^{+} v$ with Hadronic B tags, Phys. Rev. D77 (2008) 011107, [arXiv:0708.2260].

[156] BABAR Collaboration, B. Aubert et. al., A Search for $B^{+} \rightarrow \ell^{+} v_{\ell}$ Recoiling Against $B^{-} \rightarrow D^{0} \ell^{-} \bar{v} X$, arXiv:0809.4027.

[157] K. Ikado et. al., Evidence of the purely leptonic decay $B^{-} \rightarrow \tau^{-} \bar{v}_{\tau}$, Phys. Rev. Lett. 97 (2006) 251802, [hep-ex/0604018].

[158] Belle Collaboration, I. Adachi et. al., Measurement of $B^{-} \rightarrow \tau^{-} \bar{v}_{\tau}$ Decay With a Semileptonic Tagging Method, arXiv:0809.3834.

[159] V. Tisserand, CKM fits as of winter 2009 and sensitivity to New Physics, arXiv:0905.1572.

[160] U. Nierste, S. Trine, and S. Westhoff, Charged-Higgs effects in a new $B \rightarrow D \tau \nu$ differential decay distribution, Phys. Rev. D78 (2008) 015006, [arXiv: 0801 . 4938].

[161] W.-S. Hou, Enhanced charged Higgs boson effects in $B \rightarrow \tau \bar{v}_{\tau}, \mu \bar{v}_{\mu}$ and $b \rightarrow \tau \bar{v}+X$, Phys. Rev. D48 (1993) 2342-2344.

[162] A. G. Akeroyd and S. Recksiegel, The effect of $H^{ \pm}$on $B^{ \pm} \rightarrow \tau^{ \pm} v_{\tau}$ and $B^{ \pm} \rightarrow \mu^{ \pm} v_{\mu}$, J. Phys. G29 (2003) 2311-2317, [hep-ph/0306037].

[163] G. Isidori and P. Paradisi, Hints of large $\tan \beta$ in flavour physics, Phys. Lett. B639 (2006) 499-507, [hep-ph/0605012].

[164] FlaviaNet Working Group on Kaon Decays Collaboration, M. Antonelli et. al., Precision tests of the Standard Model with leptonic and semileptonic kaon decays, arXiv:0801.1817.

[165] G. Barenboim, P. Paradisi, O. Vives, E. Lunghi, and W. Porod, Light charged Higgs at the beginning of the LHC era, JHEP 04 (2008) 079, [arXiv : 0712 . 3559].

[166] J. Ellis, Outlook for Charged Higgs Physics, arXiv:0901.1120.

[167] G. Isidori, F. Mescia, and C. Smith, Light-quark loops in $K \rightarrow \pi v \bar{v}$, Nucl. Phys. B718 (2005) 319-338, [hep-ph/0503107].

[168] F. Mescia and C. Smith, Improved estimates of rare $K$ decay matrix-elements from $K_{l 3}$ decays, Phys. Rev. D76 (2007) 034017, [arXiv: 0705.2025$].$

[169] G. Buchalla, G. D’Ambrosio, and G. Isidori, Extracting short-distance physics from $K_{L, S} \rightarrow \pi^{0} e^{+} e^{-}$ decays, Nucl. Phys. B672 (2003) 387-408, [hep-ph/ 0308008$].$

[170] G. Isidori, C. Smith, and R. Unterdorfer, The rare decay $K_{L} \rightarrow \pi^{0} \mu^{+} \mu^{-}$within the SM, Eur. Phys. J. C36 (2004) 57-66, [hep-ph / 0404127$].$

[171] S. Friot, D. Greynat, and E. De Rafael, Rare kaon decays revisited, Phys. Lett. $\mathbf{B 5 9 5}$ (2004) 301-308, [hep-ph/0404136].

[172] F. Mescia, C. Smith, and S. Trine, $K_{L} \rightarrow \pi^{0} e^{+} e^{-}$and $K_{L} \rightarrow \pi^{0} \mu^{+} \mu^{-}:$A binary star on the stage of flavor physics, JHEP 08 (2006) 088, [hep-ph / 0606081$].$

[173] J. Prades, ChPT Progress on Non-Leptonic and Radiative Kaon Decays, PoS KAON (2008) 022, [arXiv:0707.1789]. 
[174] A. J. Buras, M. E. Lautenbacher, M. Misiak, and M. Munz, Direct CP violation in $K_{L} \rightarrow \pi^{0} e^{+} e^{-}$ beyond leading logarithms, Nucl. Phys. B423 (1994) 349-383, [hep-ph/940234 7].

[175] P. Colangelo, F. De Fazio, P. Santorelli, and E. Scrimieri, Rare $B \rightarrow K^{*} v \bar{v}$ decays at B factories, Phys. Lett. B395 (1997) 339-344, [hep-ph/ 9610297$].$

[176] G. Buchalla, G. Hiller, and G. Isidori, Phenomenology of non-standard Z couplings in exclusive semileptonic $b \rightarrow$ s transitions, Phys. Rev. D63 (2001) 014015, [hep-ph/ 0006136 ].

[177] W. Altmannshofer, A. J. Buras, D. M. Straub, and M. Wick, New strategies for New Physics search in $B \rightarrow K^{*} v \bar{v}, B \rightarrow K v \bar{v}$ and $B \rightarrow X_{S} v \bar{v}$ decays, JHEP 04 (2009) 022, [arXiv : 0902.0160 ].

[178] M. Bartsch, M. Beylich, G. Buchalla, and D. N. Gao, Precision Flavour Physics with $B \rightarrow K v \bar{v}$ and $B \rightarrow K l^{+} l^{-}$, arXiv:0909.1512.

[179] J. F. Kamenik and C. Smith, Tree-level contributions to the rare decays $B^{+} \rightarrow \pi^{+} v \bar{v}, B^{+} \rightarrow K^{+} v \bar{v}$, and $B^{+} \rightarrow K^{*+} v \bar{v}$ in the Standard Model, arXiv:0908.1174.

[180] A. J. Buras, M. Jamin, and M. E. Lautenbacher, The Anatomy of $\varepsilon^{\prime} / \varepsilon$ beyond leading logarithms with improved hadronic matrix elements, Nucl. Phys. B408 (1993) 209-285, [hep-ph/9303284].

[181] M. Ciuchini, E. Franco, G. Martinelli, and L. Reina, The $\Delta S=1$ effective Hamiltonian including next-to- leading order QCD and QED corrections, Nucl. Phys. B415 (1994) 403-462, [hep-ph/9304257].

[182] NA48 Collaboration, J. R. Batley et. al., A precision measurement of direct CP violation in the decay of neutral kaons into two pions, Phys. Lett. B544 (2002) 97-112, [hep-ex/ 0208009 ].

[183] KTeV Collaboration, A. Alavi-Harati et. al., Measurements of Direct CP Violation, CPT Symmetry, and Other Parameters in the Neutral Kaon System, Phys. Rev. D67 (2003) 012005, [hep-ex/0208007].

[184] KTeV Collaboration, E. T. Worcester, The Final Measurement of $\varepsilon^{\prime} / \varepsilon$ from KTeV, arXiv:0909.2555.

[185] M. Blanke, A. J. Buras, S. Recksiegel, C. Tarantino, and S. Uhlig, Correlations between $\varepsilon^{\prime} / \varepsilon$ and rare $K$ decays in the littlest Higgs model with T-parity, JHEP 06 (2007) 082, [arXiv:0704.3329].

[186] A. J. Buras and M. Jamin, $\varepsilon^{\prime} / \varepsilon$ at the NLO: 10 years later, JHEP 01 (2004) 048, [hep-ph/0306217].

[187] A. Pich, $\varepsilon^{\prime} / \varepsilon$ in the standard model: Theoretical update, hep-ph/0410215.

[188] BABAR Collaboration, B. Aubert et. al., Evidence for $D^{0}-\bar{D}^{0}$ mixing, Phys. Rev. Lett. 98 (2007) 211802, [hep-ex/0703020].

[189] Belle Collaboration, M. Staric et. al., Evidence for $D^{0}-\bar{D}^{0}$ Mixing, Phys. Rev. Lett. 98 (2007) 211803, [hep-ex/0703036].

[190] BELLE Collaboration, K. Abe et. al., Measurement of $D^{0}-\bar{D}^{0}$ mixing in $D^{0} \rightarrow K_{s} \pi^{+} \pi^{-}$decays, Phys. Rev. Lett. 99 (2007) 131803, [arXiv:0 704 . 1000].

[191] S. Bianco, F. L. Fabbri, D. Benson, and I. Bigi, A Cicerone for the Physics of Charm, Riv. Nuovo Cim. 26N7 (2003) 1-200, [hep-ex/0309021].

[192] G. Burdman and I. Shipsey, $D^{0}-\overline{D^{0}}$ Mixing and Rare Charm Decays, Ann. Rev. Nucl. Part. Sci. 53 (2003) 431-499, [hep-ph/ 0310076$].$ 
[193] I. I. Y. Bigi and N. G. Uraltsev, $D^{0}-\bar{D}^{0}$ oscillations as a probe of quark-hadron duality, Nucl. Phys. B592 (2001) 92-106, [hep-ph/ 0005089 ].

[194] A. F. Falk, Y. Grossman, Z. Ligeti, Y. Nir, and A. A. Petrov, The $D^{0}-\bar{D}^{0}$ mass difference from a dispersion relation, Phys. Rev. D69 (2004) 114021, [hep-ph/ 0402204 ].

[195] A. F. Falk, Y. Grossman, Z. Ligeti, and A. A. Petrov, SU(3) breaking and $D^{0}-\bar{D}^{0}$ mixing, Phys. Rev. D65 (2002) 054034, [hep-ph/0110317].

[196] Y. Grossman, A. L. Kagan, and Y. Nir, New physics and CP violation in singly Cabibbo suppressed D decays, Phys. Rev. D75 (2007) 036008, [hep-ph / 0609178$].$

[197] Y. Nir, Lessons from BaBar and Belle measurements of D - $\bar{D}$ mixing parameters, JHEP 05 (2007) 102, [hep-ph/0703235].

[198] K. Blum, Y. Grossman, Y. Nir, and G. Perez, Combining $K-\bar{K}$ mixing and $D-\bar{D}$ mixing to constrain the flavor structure of new physics, Phys. Rev. Lett. 102 (2009) 211802, [arXiv:0903.2118].

[199] Y. Grossman, Y. Nir, and G. Perez, Testing New Indirect CP Violation, Phys. Rev. Lett. 103 (2009) 071602, [arXiv:0904.0305].

[200] I. I. Bigi, M. Blanke, A. J. Buras, and S. Recksiegel, CP Violation in $D^{0}-\bar{D}^{0}$ Oscillations: General Considerations and Applications to the Littlest Higgs Model with T-Parity, JHEP 07 (2009) 097, [arXiv:0904.1545].

[201] A. S. Kronfeld, Non-Standard Physics in Leptonic and Semileptonic Decays of Charmed Mesons, PoS LATTICE2008 (2008) 282, [arXiv: 0812 . 2030].

[202] M. C. Gonzalez-Garcia and M. Maltoni, Phenomenology with Massive Neutrinos, Phys. Rept. 460 (2008) 1-129, [arXiv: 0704.1800$]$.

[203] G. Altarelli, Theoretical Models of Neutrino Mixing: Recent Developments, arXiv:0905.2350.

[204] A. Masiero, P. Paradisi, and R. Petronzio, Probing new physics through $\mu-e$ universality in $K \rightarrow l v$, Phys. Rev. D74 (2006) 011701, [hep-ph / 0511289 ].

[205] A. Masiero, P. Paradisi, and R. Petronzio, Anatomy and Phenomenology of the Lepton Flavor Universality in SUSY Theories, JHEP 11 (2008) 042, [arXiv: 0807 . 4721].

[206] MEGA Collaboration, M. L. Brooks et. al., New limit for the family-number non-conserving decay $\mu^{+} \rightarrow e^{+} \gamma$, Phys. Rev. Lett. 83 (1999) 1521-1524, [hep-ex/9905013].

[207] S. Yamada, Search for the lepton flavor violating decay $\mu \rightarrow$ e $\gamma$ in the MEG experiment, Nucl. Phys. Proc. Suppl. 144 (2005) 185-188.

[208] PRISM/PRIME working group Collaboration, Y. Mori et. al., LOI at J-PARC 50-GeV PS, LOI-25, http://psuxl.kek.jp/jhf-np/LOIlist/LOIlist.html.

[209] SINDRUM II. Collaboration, C. Dohmen et. al., Test of lepton flavor conservation in $\mu \rightarrow e$ conversion on titanium, Phys. Lett. B317 (1993) 631-636.

[210] M. Blanke, A. J. Buras, B. Duling, A. Poschenrieder, and C. Tarantino, Charged Lepton Flavour Violation and $(g-2)_{\mu}$ in the Littlest Higgs Model with T-Parity: a clear Distinction from Supersymmetry, JHEP 05 (2007) 013, [hep-ph / 0702136$].$

[211] F. del Aguila, J. I. Illana, and M. D. Jenkins, Precise limits from lepton flavour violating processes on the Littlest Higgs model with T-parity, JHEP 01 (2009) 080, [arXiv : 0811 . 2891]. 
[212] J. R. Ellis, J. Hisano, M. Raidal, and Y. Shimizu, A new parametrization of the seesaw mechanism and applications in supersymmetric models, Phys. Rev. D66 (2002) 115013, [hep-ph/0206110].

[213] E. Arganda and M. J. Herrero, Testing supersymmetry with lepton flavor violating $\tau$ and $\mu$ decays, Phys. Rev. D73 (2006) 055003, [hep-ph/ 0510405$].$

[214] A. Brignole and A. Rossi, Anatomy and phenomenology of $\mu \rightarrow \tau$ lepton flavour violation in the MSSM, Nucl. Phys. B701 (2004) 3-53, [hep-ph/ 0404211$].$

[215] P. Paradisi, Higgs-mediated $\tau \rightarrow \mu$ and $\tau \rightarrow e$ transitions in II Higgs doublet model and supersymmetry, JHEP 02 (2006) 050, [hep-ph / 0508054 ].

[216] P. Paradisi, Higgs-mediated $e \rightarrow \mu$ transitions in II Higgs doublet model and supersymmetry, JHEP 08 (2006) 047, [hep-ph/ 0601100$].$

[217] T. Feldmann, Neutrinos and Lepton Flavour Violation, arXiv:0909.1691.

[218] M. Pospelov and A. Ritz, Electric dipole moments as probes of new physics, Annals Phys. 318 (2005) 119-169, [hep-ph/0504231].

[219] C. A. Baker et. al., An improved experimental limit on the electric dipole moment of the neutron, Phys. Rev. Lett. 97 (2006) 131801, [hep-ex/0602020].

[220] B. C. Regan, E. D. Commins, C. J. Schmidt, and D. DeMille, New limit on the electron electric dipole moment, Phys. Rev. Lett. 88 (2002) 071805.

[221] J. Hisano, M. Nagai, and P. Paradisi, A Complete Analysis of 'Flavored' Electric Dipole Moments in Supersymmetric Theories, arXiv:0812.4283.

[222] J. Prades, Standard Model Prediction of the Muon Anomalous Magnetic Moment, arXiv:0909.2546.

[223] Muon g-2 Collaboration, G. W. Bennett et. al., Measurement of the Positive Muon Anomalous Magnetic Moment to 0.7 ppm, Phys. Rev. Lett. 89 (2002) 101804, [hep-ex/ 0208001 ].

[224] Muon g-2 Collaboration, G. W. Bennett et. al., Measurement of the negative muon anomalous magnetic moment to 0.7-ppm, Phys. Rev. Lett. 92 (2004) 161802, [hep-ex/ 0401008 ].

[225] M. Passera, Status of the standard model prediction of the muon g-2, Nucl. Phys. Proc. Suppl. 155 (2006) 365-368, [hep-ph/0509372].

[226] M. Passera, The standard model prediction of the muon anomalous magnetic moment, J. Phys. G31 (2005) R75-R94, [hep-ph/0411168].

[227] G. Isidori, F. Mescia, P. Paradisi, and D. Temes, Flavour physics at large $\tan \beta$ with a Bino-like LSP, Phys. Rev. D75 (2007) 115019, [hep-ph/ 0703035$].$

[228] S. R. Choudhury, A. S. Cornell, A. Deandrea, N. Gaur, and A. Goyal, Lepton flavour violation in the little Higgs model, Phys. Rev. D75 (2007) 055011, [hep-ph/ 0612327$].$

[229] M. Passera, W. J. Marciano, and A. Sirlin, The Muon g-2 and the bounds on the Higgs boson mass, Phys. Rev. D78 (2008) 013009, [arXiv: 0804 .1142].

[230] M. Passera, W. J. Marciano, and A. Sirlin, The muon g-2 discrepancy: errors or new physics?, AIP Conf. Proc. 1078 (2009) 378-381, [arXiv: 0809.4062$].$

[231] E. De Rafael, Present Status of the Muon Anomalous Magnetic Moment, Nucl. Phys. Proc. Suppl. 186 (2009) 211-217, [arXiv:0809.3085]. 
[232] F. Jegerlehner and A. Nyffeler, The Muon g-2, Phys. Rept. 477 (2009) 1-110, [arXiv:0902.3360].

[233] Y. Grossman, Y. Nir, J. Thaler, T. Volansky, and J. Zupan, Probing Minimal Flavor Violation at the LHC, Phys. Rev. D76 (2007) 096006, [arXiv: 0706.1845$].$

[234] J. L. Feng, C. G. Lester, Y. Nir, and Y. Shadmi, The Standard Model and Supersymmetric Flavor Puzzles at the Large Hadron Collider, Phys. Rev. D77 (2008) 076002, [arXiv: 0712.0674 ].

[235] K. Agashe et. al., LHC Signals for Warped Electroweak Neutral Gauge Bosons, Phys. Rev. D76 (2007) 115015, [arXiv:0709.0007].

[236] K. Agashe, A. Belyaev, T. Krupovnickas, G. Perez, and J. Virzi, LHC signals from warped extra dimensions, Phys. Rev. D77 (2008) 015003, [hep-ph / 0612015 ].

[237] K. Agashe, G. Perez, and A. Soni, Collider Signals of Top Quark Flavor Violation from a Warped Extra Dimension, Phys. Rev. D75 (2007) 015002, [hep-ph / 0606293 ].

[238] K. Agashe, S. Gopalakrishna, T. Han, G.-Y. Huang, and A. Soni, LHC Signals for Warped Electroweak Charged Gauge Bosons, arXiv:0810.1497.

[239] G. Hiller and Y. Nir, Measuring Flavor Mixing with Minimal Flavor Violation at the LHC, JHEP 03 (2008) 046, [arXiv:0802.0916].

[240] J. Drobnak, S. Fajfer, and J. F. Kamenik, Signatures of NP models in top FCNC decay $t \rightarrow c(u) l^{+} l^{-}$ , JHEP 03 (2009) 077, [arXiv: 0812.0294].

[241] CKMfitter Group Collaboration, J. Charles et. al., CP violation and the CKM matrix: Assessing the impact of the asymmetric B factories, Eur. Phys. J. C41 (2005) 1-131, [hep-ph/ 0406184 ]. Updates available on http://ckmfitter.in2p3.fr/.

[242] UTfit Collaboration, M. Bona et. al., The UTfit collaboration report on the unitarity triangle beyond the standard model: Spring 2006, Phys. Rev. Lett. 97 (2006) 151803, [hep-ph / 0605213 ]. Updates available on http: / / www . utfit.org.

[243] M. Ciuchini et. al., 2000 CKM-triangle analysis: A critical review with updated experimental inputs and theoretical parameters, JHEP 07 (2001) 013, [hep-ph / 0012308 ]. see also www.utfit.org.

[244] A. Hocker, H. Lacker, S. Laplace, and F. Le Diberder, A new approach to a global fit of the CKM matrix, Eur. Phys. J. C21 (2001) 225-259, [hep-ph / 0104062 ]. see also ckmfitter.in2p3.fr.

[245] UTfit Collaboration, M. Bona et. al., The UTfit collaboration report on the status of the unitarity triangle beyond the standard model. I: Model-independent analysis and minimal flavour violation, JHEP 03 (2006) 080, [hep-ph/ 0509219 ].

[246] M. Bartsch, G. Buchalla, and C. Kraus, $B \rightarrow V_{L} V_{L}$ Decays at Next-to-Leading Order in QCD, arXiv:0810.0249.

[247] T. Goto, N. Kitazawa, Y. Okada, and M. Tanaka, Model independent analysis of $B-\bar{B}$ mixing and CP violation in B decays, Phys. Rev. D53 (1996) 6662-6665, [hep-ph/9506311].

[248] G. Buchalla and A. J. Buras, $\sin 2 \beta$ from $K \rightarrow \pi v \bar{v}$, Phys. Lett. B333 (1994) 221-227, [hep-ph/9405259].

[249] A. J. Buras, F. Parodi, and A. Stocchi, The CKM matrix and the unitarity triangle: Another look, JHEP 01 (2003) 029, [hep-ph/ 0207101$].$

[250] W. Altmannshofer, A. J. Buras, and D. Guadagnoli, The MFV limit of the MSSM for low $\tan \beta$ : meson mixings revisited, JHEP 11 (2007) 065, [hep-ph / 0703200 ]. 
[251] A. J. Buras, M. Jamin, and P. H. Weisz, Leading and next-to-leading QCD corrections to $\varepsilon_{K}$ parameter and $B^{0}-\bar{B}_{0}$ mixing in the presence of a heavy top quark, Nucl. Phys. B347 (1990) 491-536.

[252] S. Herrlich and U. Nierste, Enhancement of the $K_{L}-K_{S}$ mass difference by short distance QCD corrections beyond leading logarithms, Nucl. Phys. B419 (1994) 292-322, [hep-ph/9310311].

[253] S. Herrlich and U. Nierste, Indirect CP violation in the neutral kaon system beyond leading logarithms, Phys. Rev. D52 (1995) 6505-6518, [hep-ph/9507262].

[254] S. Herrlich and U. Nierste, The Complete $|\Delta S|=2$ Hamiltonian in the Next-To-Leading Order, Nucl. Phys. B476 (1996) 27-88, [hep-ph/9604330].

[255] A. J. Buras and D. Guadagnoli, Correlations among new CP violating effects in $\Delta F=2$ observables, Phys. Rev. D78 (2008) 033005, [arXiv: 0805 .3887].

[256] C. Aubin, J. Laiho, and R. S. Van de Water, The neutral kaon mixing parameter $B_{K}$ from unquenched mixed-action lattice $Q C D$, arXiv:0905.3947.

[257] RBC Collaboration, D. J. Antonio et. al., Neutral kaon mixing from 2+1 flavor domain wall QCD, Phys. Rev. Lett. 100 (2008) 032001, [hep-ph/ 0702042$].$

[258] A. J. Buras and D. Guadagnoli, On the consistency between the observed amount of CP violation in the $K^{-}$and $B_{d}$-systems within minimal flavor violation, Phys. Rev. D79 (2009) 053010, [arXiv:0901.2056].

[259] K. Anikeev et. al., B physics at the Tevatron: Run II and beyond, hep-ph/ 0201071.

[260] E. A. Andriyash, G. G. Ovanesyan, and M. I. Vysotsky, Difference of $\tilde{\varepsilon}$ and $\varepsilon$ in fitting the parameters of CKM matrix, Phys. Lett. B599 (2004) 253-259, [hep-ph/ 0310314$].$

[261] E. A. Andriyash, G. G. Ovanesyan, and M. I. Vysotsky, The value of $B(K)$ from the experimental data on CP-violation in K-mesons and up-to-date values of CKM matrix parameters, Phys. Atom. Nucl. 69 (2006) 286-292, [hep-ph/0502111].

[262] Particle Data Group Collaboration, C. Amsler et. al., Review of particle physics, Phys. Lett. B667 (2008) 1 .

[263] E. Lunghi and A. Soni, Possible Indications of New Physics in $B_{d}$-mixing and in $\sin (2 \beta)$ Determinations, Phys. Lett. B666 (2008) 162-165, [arXiv: 0803.4340 ].

[264] E. Lunghi and A. Soni, Hints for the scale of new CP-violating physics from B-CP anomalies, JHEP 08 (2009) 051, [arXiv:0903.5059].

[265] M. Ciuchini, M. Pierini, and L. Silvestrini, The effect of penguins in the $B_{d} \rightarrow J / \psi K^{0} C P$ asymmetry, Phys. Rev. Lett. 95 (2005) 221804, [hep-ph/ 0507290 ].

[266] S. Faller, M. Jung, R. Fleischer, and T. Mannel, The Golden Modes $B^{0} \rightarrow J / \psi K_{S, L}$ in the Era of Precision Flavour Physics, arXiv:0809.0842.

[267] M. Gronau and J. L. Rosner, Doubly CKM-suppressed corrections to CP asymmetries in $B^{0} \rightarrow J / \psi K^{0}$, Phys. Lett. B672 (2009) 349-353, [arXiv : 0812 . 4796].

[268] A. J. Buras, F. Schwab, and S. Uhlig, Waiting for precise measurements of $K^{+} \rightarrow \pi^{+} v \bar{v}$ and $K_{L} \rightarrow \pi^{0} v \bar{v}$, Rev. Mod. Phys. 80 (2008) 965-1007, [hep-ph/ 0405132 ].

[269] C. Smith, Theory review on rare K decays: Standard model and beyond, hep-ph/0608343.

[270] G. Isidori, KAON 2007: Conference Summary, PoS KAON (2008) 064, [arXiv : 0709.2438 ]. 
[271] C. Smith, Long-distance effects in rare and radiative K decays, arXiv: 0908.3434.

[272] M. Gorbahn, NNLO contributions to $\varepsilon_{K}$ and rare kaon decays, arXiv: 0909.2221.

[273] E949 Collaboration, A. V. Artamonov et. al., New measurement of the $K^{+} \rightarrow \pi^{+} v \bar{v}$ branching ratio, Phys. Rev. Lett. 101 (2008) 191802, [arXiv: 0808.2459$].$

[274] E391a Collaboration, J. K. Ahn et. al., Search for the Decay $K_{L}^{0} \rightarrow \pi^{0} v \bar{v}$, Phys. Rev. Lett. 100 (2008) 201802, [arXiv:0712.4164].

[275] A. J. Buras and R. Fleischer, Bounds on the unitarity triangle, $\sin 2 \beta$ and $K \rightarrow \pi \nu \bar{v}$ decays in models with minimal flavor violation, Phys. Rev. D64 (2001) 115010, [hep-ph / 0104238 ].

[276] M. Blanke et. al., Rare and CP-violating K and B decays in the Littlest Higgs model with T-parity, JHEP 01 (2007) 066, [hep-ph / 0610298 ].

[277] A. J. Buras, Relations between $\Delta M_{s, d}$ and $B_{s, d} \rightarrow \mu \bar{\mu}$ in models with minimal flavour violation, Phys. Lett. B566 (2003) 115-119, [hep-ph/0303060].

[278] CDF Collaboration, T. Aaltonen et. al., Search for $B_{s}^{0} \rightarrow \mu^{+} \mu^{-}$and $B_{d}^{0} \rightarrow \mu^{+} \mu^{-}$decays with $2 \mathrm{fb}^{-1}$ of p $\bar{p}$ collisions, Phys. Rev. Lett. 100 (2008) 101802, [arXiv: 0712.1708 ].

[279] D0 Collaboration, V. M. Abazov et. al., Search for $B_{s} \rightarrow \mu^{+} \mu^{-}$at D0, Phys. Rev. D76 (2007) 092001, [arXiv:0707.3997].

[280] S. R. Choudhury and N. Gaur, Dileptonic decay of $B_{s}$ meson in SUSY models with large tan(beta), Phys. Lett. B451 (1999) 86-92, [hep-ph/9810307].

[281] K. S. Babu and C. F. Kolda, Higgs mediated $B^{0} \rightarrow \mu^{+} \mu^{-}$in minimal supersymmetry, Phys. Rev. Lett. 84 (2000) 228-231, [hep-ph/9909476].

[282] A. J. Buras, P. H. Chankowski, J. Rosiek, and L. Slawianowska, $\Delta M_{d, s}, B_{d, s}^{0} \rightarrow \mu^{+} \mu^{-}$and $B \rightarrow X_{s} \gamma$ in supersymmetry at large $\tan \beta$, Nucl. Phys. B659 (2003) 3, [hep-ph/0210145].

[283] A. J. Buras, P. H. Chankowski, J. Rosiek, and L. Slawianowska, Correlation between $\Delta M_{s}$ and $B_{s, d}^{0} \rightarrow \mu^{+} \mu^{-}$in supersymmetry at large $\tan \beta$, Phys. Lett. B546 (2002) 96-107, [hep-ph/0207241].

[284] G. Isidori and A. Retico, $B_{s, d} \rightarrow l^{+} l^{-}$and $K_{L} \rightarrow l^{+} l^{-}$in SUSY models with non-minimal sources of flavour mixing, JHEP 09 (2002) 063, [hep-ph / 0208159 ].

[285] M. S. Carena, A. Menon, R. Noriega-Papaqui, A. Szynkman, and C. E. M. Wagner, Constraints on B and Higgs physics in minimal low energy supersymmetric models, Phys. Rev. D74 (2006) 015009, [hep-ph/0603106].

[286] M. Gorbahn, S. Jager, U. Nierste, and S. Trine, The supersymmetric Higgs sector and $B-\bar{B}$ mixing for large $\tan \beta$, arXiv:0901.2065.

[287] P. H. Chankowski and J. Rosiek, Supersymmetry (at large $\tan \beta$ ) and flavor physics, Acta Phys. Polon. B33 (2002) 2329-2354, [hep-ph / 0207242 ].

[288] A. Dedes and A. Pilaftsis, Resummed effective Lagrangian for Higgs-mediated FCNC interactions in the CP-violating MSSM, Phys. Rev. D67 (2003) 015012, [hep-ph/0209306].

[289] A. Dedes, The Higgs penguin and its applications: An Overview, Mod. Phys. Lett. A18 (2003) 2627-2644, [hep-ph/0309233].

[290] G. Hiller, Y. Hochberg, and Y. Nir, Flavor Changing Processes in Supersymmetric Models with Hybrid Gauge- and Gravity-Mediation, JHEP 03 (2009) 115, [arXiv : 0812 . 0511]. 
[291] S. Faller, R. Fleischer, and T. Mannel, Precision Physics with $B_{s}^{0} \rightarrow J / \psi \phi$ at the LHC: The Quest for New Physics, Phys. Rev. D79 (2009) 014005, [arXiv: 0810 . 4248].

[292] A. Lenz and U. Nierste, Theoretical update of $B_{S}-\bar{B}_{s}$ mixing, JHEP 06 (2007) 072 , [hep-ph/0612167].

[293] UTfit Collaboration, M. Bona et. al., First Evidence of New Physics in $b \rightarrow s$ Transitions, arXiv:0803.0659.

[294] CDF Collaboration, G. Brooijmans, Mixing and CP Violation at the Tevatron, arXiv:0808.0726.

[295] A. J. Lenz, Search for new physics in $B_{s}$-mixing, arXiv:0808.1944.

[296] Z. Ligeti, M. Papucci, and G. Perez, Implications of the measurement of the $B_{s}^{0}-\bar{B}_{s}^{0}$ mass difference, Phys. Rev. Lett 97 (2006) 101801, [hep-ph/ 0604112 ].

[297] M. Blanke, Insights from the Interplay of $K \rightarrow \pi v \bar{v}$ and $\varepsilon_{K}$ on the New Physics Flavour Structure, arXiv:0904.2528.

[298] R. Dermisek and S. Raby, Bi-large neutrino mixing and CP violation in an SO(10) SUSY GUT for fermion masses, Phys. Lett. B622 (2005) 327-338, [hep-ph/0507045].

[299] M. Albrecht, W. Altmannshofer, A. J. Buras, D. Guadagnoli, and D. M. Straub, Challenging SO(10) SUSY GUTs with family symmetries through FCNC processes, JHEP 10 (2007) 055, [arXiv:0707.3954].

[300] W. Altmannshofer, D. Guadagnoli, S. Raby, and D. M. Straub, SUSY GUTs with Yukawa unification: a go/no-go study using FCNC processes, Phys. Lett. B668 (2008) 385-391, [arXiv: 0801 . 4363 ].

[301] C. Quigg, Unanswered Questions in the Electroweak Theory, arXiv:0905.3187. 